\title{
A Toolbox of Solid-State NMR Experiments for the Characterization of Soft Organic Nanomaterials
}

\author{
Lasse Arnt Straas $\varnothing,{ }^{1}$ Qasim Saleem, ${ }^{2}$ and Michael Ryan Hansen ${ }^{3}$ \\ ${ }^{1}$ Interdisciplinary Nanoscience Center (iNANO), Aarhus University, Gustav Wieds Vej 14, \\ DK-8000 Aarhus C, Denmark \\ ${ }^{2}$ Physical Science and Engineering Division, Solar and Photovoltaics Engineering \\ Research Center, King Abdullah University of Science and Technology, Thuwal 23955- \\ 6900, Kingdom of Saudi Arabia
}

${ }^{3}$ Institute of Physical Chemistry, Westfälische Wilhelms-Universität Münster, Corrensstr. 28/30, D-48149 Münster, Germany

Key Words: Organic solids, 2D NMR techniques, NMR interactions, NMR theory and Hamiltonians, average Hamiltonian theory, product operators, polymers, natural silk, $\pi$ conjugated polymers, graphene-related materials 


\begin{abstract}
Determining how organic molecules self-assemble into a solid material is a challenging and demanding task if a single crystal of the material cannot be produced. Solid-state NMR spectroscopy offers access to such molecular details via an appropriate selection of techniques. This report gives a selected overview of $1 \mathrm{D}$ and $2 \mathrm{D}$ solid-state NMR techniques for elucidating the structure of soft organic solids. We focus on how the solidstate NMR techniques are designed from the perspective of the different nuclear interactions, using average Hamiltonian theory and product operators. We also introduce recent methods for quantification and reduction of experimental artifacts. Finally, we highlight how the solid-state NMR techniques can be applied to soft organic materials by reviewing recent applications to semi-crystalline polymers, $\pi$-conjugated polymers, natural silk, and graphene-related materials.
\end{abstract}

\title{
1. INTRODUCTION
}

Soft organic materials are those that can be easily deformed by thermal or mechanical stresses, and experience thermal fluctuations at room temperature. These include polymers, foams, gels, granular materials, liquid crystals and colloids, as well as most soft biological materials [1]. These materials may also be classified as macromolecules or supramolecular systems, and their specific function is often established via self assembly [2]. This process is driven by the incompatibility of the different chemical building blocks, like that of a polymer backbone and its attached aliphatic side chains, or non-covalent interactions, such as hydrogen bonds, ionic forces, and $\pi-\pi$-interactions $[3,4]$. Soft organic 
materials generally exhibit attractive mechanical properties. This allows for their use in a wide variety of applications. For example, self-assembled organic materials have shown promising use as semi-conductors [5, 6]. Organic semi-conductors benefit from (i) extended $\pi$-conjugation due to $\mathrm{sp}^{2}$-hybridized carbon systems and (ii) solubility in organic solvents via strategically introduced solubilizing aliphatic side chains $[7,8]$. These advantages make organic materials amenable to solution processing techniques, which have the potential to create thin and light-weight electronic devices of the future [9]. However, a general property of soft organic materials is that they rarely crystallize into a single crystal. Typically, crystallization leads the formation of domains with high and low structural order, making these materials semi-crystalline. Well-known examples of soft organic materials displaying semi crystallinity are synthetic polymers or polymer systems composed of different repeat units such as polymer blends or copolymers. Similarly, biomacromolecules, like proteins, are also composed of well-ordered regions containing ahelices or $\beta$-sheets that are interlinked by conformationally disordered chain segments [10].

One of the major challenges identified in the area of macromolecular science is the structural characterization of soft organic materials at the molecular level [11]. Besides semi-crystallinity of the macromolecular systems as discussed above, the challenge also arises from the intrinsic heterogeneity present within them. This heterogeneity arises from such aspects as chain length, stereo- and regio-chemistry, architectural perfection, and copolymer composition. From a molecular point-of-view, solid-state NMR spectroscopy performed under high-resolution conditions, utilizing magic-angle spinning (MAS) and highpower ${ }^{1} \mathrm{H}$ decoupling, offers site-specific information and thereby has access to detailed 
information about molecular order and local packing arrangement in soft organic materials. This information can be established by probing the local molecular environments of proton $\left({ }^{1} \mathrm{H}\right)$ and carbon $\left({ }^{13} \mathrm{C}\right)$ species as reflected in their isotropic chemical shift $\left(\delta_{\text {iso }}\right)$ values. The molecular packing of self-assembled $\pi$-conjugated systems is mainly observed as a nucleus independent shift to higher or lower chemical shift on the order of $1-10 \mathrm{ppm}$ as compared to the $\delta_{\text {iso }}$ observed in solution NMR $[12,13]$. This makes ${ }^{1} \mathrm{H}$ NMR the obvious choice for probing this type of interaction, since ${ }^{1} \mathrm{H}$ has a much lower chemical shift dispersion (ca. $15 \mathrm{ppm}$ ) as compared to ${ }^{13} \mathrm{C}$ (ca. $220 \mathrm{ppm}$ ) [14]. Moreover, hydrogen bonding, potentially designed to form between different molecules or segments of the macromolecular structure [15], also shifts the $\delta_{\text {iso }}$ compared to that observed in a nonhydrogen bonding solvent by solution NMR. If a hydrogen bond is formed, the ${ }^{1} \mathrm{H}$ chemical shift of the involved proton strongly depends on the bond length. Hence, hydrogen-bonded protons are typically observed in the spectral range between 8 and 20 ppm, reflecting the strength of hydrogen bond $[16,17]$ making them easy to identify in ${ }^{1} \mathrm{H}$ MAS NMR spectra [18]. The structural order or disorder, on the other hand, is mainly reflected in the line width of the observed isotropic chemical shifts for the different chemical moieties of the probed molecule. For this reason, ${ }^{13} \mathrm{C}$ is a more appropriate nucleus for probing local order, since it has a larger chemical shift range, leading to a better chemical shift resolution. Moreover, $\delta_{\text {iso }}$ of ${ }^{13} \mathrm{C}$ is also sensitive to conformation of the methylene groups in a chain structure. This effect is known as the $Y$-gauche effect and is marked by a shift of the $\delta_{\text {iso }}$ for the ${ }^{13} \mathrm{CH}_{2}$ moiety under consideration. The effect is caused by the local conformations of the methylene groups in the $Y$-position. If the $Y$-methylene groups are in a gauche conformation with respect to the methylene groups in the a positions, this leads to a shift of 
the $\delta_{\text {iso }}$ to lower frequencies, sometimes referred to as a low-field or up-field shift, when compared to the $\delta_{\text {iso }}$ for the $\gamma$-methylene groups in trans conformation. The $\gamma$-gauche effect is well established for aliphatic structures containing long sequences of methylene groups like n-alkanes or polyethylene [19-22]. Typical shifts are in the range of 3-10 ppm, while effects due to chain packing only contribute to a minor extent of $\sim 1 \mathrm{ppm}[14]$. For ${ }^{13} \mathrm{C}$ in natural abundance, as mostly encountered in synthetic macromolecular systems, the ${ }^{13} \mathrm{C}$ nucleus is not influenced by strong homonuclear dipole-dipole couplings as in the case of ${ }^{1} \mathrm{H}$ NMR in solids, leading to a strong broadening of the observed ${ }^{1} \mathrm{H}$ resonances. In fact, the observed resonance line width differences for the isotropic chemical shifts of a number of different NMR active nuclei have been exploited for a broad range of compounds. This includes both soft organic and inorganic materials, and has led to a better understanding of the short and intermediate range order present in both semicrystalline and amorphous materials [23-26]. Thus, by combining the information available from observing ${ }^{1} \mathrm{H}$ and ${ }^{13} \mathrm{C}$ in carefully designed $1 \mathrm{D}$ and $2 \mathrm{D}$ solid-state NMR experiments, it is possible to get a more detailed picture of local packing arrangements, phase composition, and molecular order.

In this report we give an overview of $1 \mathrm{D}$ and $2 \mathrm{D}$ solid-state NMR techniques for the structural characterization of soft organic solids at the molecular level. We introduce the relevant nuclear spin interactions and use average Hamiltonian theory and product operators to show the design principles behind the solid-state NMR techniques. Finally, we highlight how the solid-state NMR techniques can be applied to soft organic materials by giving examples of recent applications to semi-crystalline polymers, $\pi$-conjugated polymers, natural silk, and graphene-related materials. 


\section{NMR BASICS}

Before describing some of the recent advances in 1D and 2D solid-state NMR techniques and give examples of their application to soft organic materials, we will briefly summarize the relevant quantum mechanics and spin interactions. We will not discuss the theory and the NMR interactions in a complete fashion, but rather give the reader an appreciation of the theoretical underpinnings of the experiments. It should be noted that all spin interactions in this section are written using Cartesian operators, in contrast to the use of spherical tensor operators often seen in the NMR literature. The interested reader can find more details in excellent NMR textbooks and reviews in Refs. [14, 27, 28].

\subsection{Theory}

In NMR spectroscopy, an ensemble of non-identical systems is studied. A convenient way of gaining an insight of this ensemble is through the use of the density operator, $\rho(t)$. Assuming no relaxation, $\rho(t)$ evolves under the influence of the Hamiltonian, $H(t)$, as formulated by the Liouville-von Neumann equation,

$$
\frac{\partial}{\partial t} \rho(t)=-i[H(t), \rho(t)] .
$$

Note that the (Liouville-von Neumann) equation is written in units of $h$. This convention will be used throughout this report. The formal solution to Eq. (0.1) is

$$
\rho(t)=U(t) \rho(0) U(t)^{\dagger}, \quad i \frac{\partial}{\partial t} U(t)=H(t) U(t)
$$

along with the Schrödinger equation for the spin propagator, $U(t)$. Finding the correct spin propagator is very challenging when $H(t)$ does not commute with itself at different times 
during the time evolution and, hence, the solution generally relies on the so-called Dyson time operator. For time periods where $H(t)$ does commute or is time-independent, it is straightforward to calculate $U(t)$. When $H(t)$ is time-independent, i.e. $H(t)=H, U(t)$ can be written as

$$
U(t)=e^{-i H t}
$$

The simple form of the spin propagator might have inspired Haeberlen and Waugh [29] to make use of the Magnus expansion to gain insight into the spin dynamics. The Magnus expansion assumes a spin propagator similar to Eq. (0.3), but with the effective Hamiltonian expanded into orders

$$
U\left(t_{c}\right)=e^{-i H_{e f f} t_{c}}, \quad H_{e f f}=H_{e f f}^{(1)}+H_{e f f}^{(2)}+\ldots
$$

Note that this is also referred to as the average Hamiltonian theory (AHT) in solid-state NMR spectroscopy. In practice, physical insight can most often be gained by calculating the first-order term (1.5) and, if necessary, also the second-order term (1.6)

$$
\begin{gathered}
H_{e f f}^{(1)}=\frac{1}{t_{c}} \int_{0}^{t_{c}} H(t) d t \\
H_{e f f}^{(2)}=\frac{-i}{2 t_{c}} \int_{0}^{t_{c}} d t_{2} \int_{0}^{t_{2}}\left[H\left(t_{2}\right), H\left(t_{1}\right)\right] d t_{1} .
\end{gathered}
$$

When making use of average Hamiltonian theory, it is important to ensure that the Hamiltonian is cyclic at time $t_{c}$, i.e. $H\left(t_{c}\right)$ does not give rise to an overall net rotation at that time point. The above first-order term is often referred to as the average Hamiltonian 
or the effective Hamiltonian to first-order. The convergence of the series expansion in Eq. (0.4) is far from trivial [30] and will not be discussed here. Average Hamiltonian theory has successfully guided the development of numerous experiments in solid-state NMR spectroscopy, as reviewed in Refs. [31, 32]. Recently, Floquet theory [33-35] has become increasingly popular in gaining insight into the coherent evolution of the spin system.

To make the series expansion in Eq. (0.4) converge faster, it is useful to transform the description into an interaction frame, where the Hamiltonian is divided into a larger and a smaller part, e.g. $H(t)=H_{0}(t)+H_{1}(t)$ with $H_{1}(t)$ being the larger term. With this approach, we can separate out the evolution due to the larger part of the Hamiltonian by writing the spin propagator as a product [29]

$$
U(t)=U_{1}(t) \tilde{U}_{0}(t)
$$

using

$$
\frac{\partial}{\partial t} U_{1}(t)=-i H_{1}(t) U_{1}(t), \quad \frac{\partial}{\partial t} \tilde{U}_{0}(t)=-i \tilde{H}_{0}(t) \tilde{U}_{0}(t), \quad \tilde{H}_{0}(t)=U_{1}^{\dagger}(t) H_{0}(t) U_{1}(t) .
$$

At cyclic times of $H_{1}(t)$, i.e. $U_{1}\left(t_{c}\right)=1$, we restrict ourselves to the calculation of the interaction frame spin propagator, $\tilde{U}_{0}\left(t_{c}\right)$.

\subsection{NMR interactions}

NMR interactions are often separated into external and internal parts. The external part consists of the static magnetic field $\left(B_{0}\right)$ that, by convention, points along the $z$-axis and a 
radio-frequency (rf) field $\left(B_{1}\right)$ that lies in the $x-y$ plane. The static magnetic field interacts with the magnetic moment of a nucleus and gives rise to a splitting of the energy levels. For a spin-1/2 nucleus, the eigenstate $\alpha$, which is chosen to point along the magnetic field, will be lower in energy than the eigenstate $\beta$ that is opposed to the magnetic field. The Zeeman Hamiltonian that signifies this interaction is written as

$$
H_{\text {Zeeman }}=\omega_{0} I_{z}, \quad \omega_{0}=\gamma_{I} B_{0}
$$

where the product of the gyromagnetic moment and the strength of the static magnetic field, $\omega_{0}=-\gamma_{I} B_{0}$, is called the Larmor frequency and $I_{z}$ is the angular momentum operator about the $z$ axis of spin $I$. If we assume the gyromagnetic moment to be larger than zero, as is the case for ${ }^{1} \mathrm{H}$ and ${ }^{13} \mathrm{C}$ spins, then the Larmor frequency is negative, resulting in a clockwise precession about the $z$ axis when the initial polarization is tilted away from equilibrium. We direct the interested reader to Refs. [36, 37] for an in-depth discussion on the signs of the frequencies and phases in NMR spectroscopy.

The energy difference between the two eigenstates is calculated as $h\left|\omega_{0}\right|$ and the Boltzmann distribution can be used to calculate the occupational difference of the energy levels and thereby the starting polarization. Using the operator formalism and invoking the high-temperature approximation, the equilibrium density operator becomes

$$
\rho\left(t_{0}\right)=Z^{-1} e^{-\beta H} ; Z^{-1}(1-\beta H)=Z^{-1}\left(1-\beta \omega_{o} I_{z}\right), \quad \beta=\frac{1}{k T},
$$

where $k$ denotes the Boltzmann constant and $Z$ denotes the partition function. According to Mehring [27], the high-temperature approximation is legitimate down to at least $1 \mathrm{~K}$. An 
observable, e.g. the magnetization, can be found as $\operatorname{Tr}[\rho M]$. The trace over $M$ vanishes, and making it convenient to work with the truncated density operator that is linear and points along the $z$ axis, $\rho \propto I_{z}$.

Viewed in the laboratory frame, the rf field can be split into a counter-clockwise and a clockwise component. In a rotating frame that matches the frequency of the clockwise component, it will appear static while the counter-clockwise component will oscillate at twice the frequency of the frame. It is convenient to describe NMR experiments in the rotating frame, and hence, in the following, only the static rf component will be taken into account. The rotating frame frequency is kept close to the Larmor frequency of the nuclei in the sample. If the rotating frame frequency exactly matches a given resonance frequency, then the effective longitudinal field will be zero in that frame.

The electronic cloud in a molecule will, on average, counteract the static magnetic field on the timescale of an NMR experiment such that each nucleus feels a specific reduced magnetic field compared to the static field. This nucleus-specific field, which can be viewed in terms of a resonance frequency, is referred to as the chemical shift when reported relative to very shielded compounds such as tetramethylsilane (TMS) or 4,4-dimethyl-4silapentane-1-sulfonic acid (DSS). The chemical shift is of utmost importance in the interpretation of NMR spectra as this allows us to deduce the chemical environment around a nucleus. For example, in ${ }^{13} \mathrm{C}$ NMR spectroscopy, the large chemical shift range of ca. $200 \mathrm{ppm}$ allows us to easily distinguish aromatics from the aliphatic moieties.

In solid-state NMR spectroscopy, the nucleus specific magnetic field further depends on the orientation of the molecule with respective to the static magnetic field. Interactions that 
depend on the relative orientation are denoted anisotropic interactions, e.g. the chemical shift anisotropy (CSA) interaction. The dependency can generally be described by a 3-by-3 matrix which can be decomposed into an isotropic, antisymmetric, and symmetric part. By definition, the symmetric part is diagonal in the principal axis system. The anti-symmetric part is often neglected, as in the case of CSA. To relate different coordinate systems, three Euler angles $(\Omega=\{\alpha, \beta, \gamma\})$ are specified, and transformations can be accomplished using Wigner matrices [27].

In liquid-state NMR spectroscopy, the anisotropic interactions cannot be seen directly due to the rapid tumbling of the molecule. In solid-state NMR, an ingenious technique called magic-angle-spinning (MAS) averages out anisotropic interactions, leaving behind mainly isotropic interactions. Unless specified, we will only consider MAS experiments in this contribution. Due to the periodic motions induced by MAS, the NMR interactions can be expended into a Fourier series in units of the rotor frequency, $\omega_{r}$, using zeroth-, firstand second-order terms. Addressing the chemical shift interaction, we can write the Hamiltonian as

$$
H_{c s}(t)=\omega_{I}(t) I_{z}=\sum_{m=-2}^{2} \omega_{I}^{(m)} e^{i m \omega_{r} t} I_{z}
$$

Under MAS conditions, $\omega_{I}^{(0)}$ is the isotropic term and $\omega_{I}^{( \pm 1)}, \omega_{I}^{( \pm 2)}$ are the anisotropic terms. We realize that an integration over a rotor period as directed by average Hamiltonian theory leaves us with only the isotropic part - similar to liquid-state NMR spectroscopy. Please note that we implicitly make use of the high-field approximation when describing 
the NMR interactions. This means that the only relevant terms are those that commute with the total angular momentum about the $z$ axis.

Another very important interaction in solid-state NMR spectroscopy is the dipole-dipole coupling interaction. For a homonuclear two-spin system, spin $I$ and spin $S$, the interaction becomes

$$
H_{I S}(t)=\omega_{I S}(t)\left(2 I_{z} S_{z}-I_{x} S_{x}-I_{y} S_{y}\right), \quad \omega_{I S}(t)=\sum_{m=-2}^{2} \omega_{I S}^{(m)} e^{i m \omega_{r} t}
$$

The m'th Fourier coefficients of the dipolar coupling is described as

$$
\omega_{I S}^{(m)}=b_{I S} d_{0,-m}^{(2)}\left(\beta_{P R}\right) d_{-m, 0}^{(2)}\left(\beta_{R L}\right) e^{i m \gamma_{P R}}, \quad b_{I S}=\frac{\mu_{0}}{4 \pi} \frac{\gamma_{I} \gamma_{S} \mathrm{~h}}{r_{I S}^{3}},
$$

with $d_{m, m}^{(2)}$ denoting the reduced Wigner elements of rank 2. $\gamma_{P R}$ is related to the rotor angle $\left(\omega_{r} t\right)$ by a rotation about the rotor axis. $b_{I S}$ is referred to as the strength or magnitude of the dipolar coupling. The specific dependency on the Euler angles relating the principle axis system $(P)$ over the rotor-fixed system $(R)$ to the laboratory frame $(L)$ has been included. In the presence of a large number of interactions, a molecular frame should be included in between $\mathrm{P}$ and $\mathrm{R}$. The dipolar coupling shows no dependency on $\alpha_{P R}$ as the interaction is axially symmetric about the internuclear axis between the two spins. For motional averaging, this symmetry can be broken and an asymmetry parameter should be introduced [14, 38].

Also, the zeroth-order Fourier term, $\omega_{I S}^{(0)}$, is zero by virtue of the magic-angle which is equal to 54.7 degrees. The effect of this setting is appreciated by realizing that 
$d_{0,0}^{(2)}(\beta)=\frac{1}{2}\left(3 \cos ^{2}(\beta)-1\right)$ [31], a Legendre polynomial: $\mathrm{P}_{2}(\cos (\beta))$. As mentioned above, the time modulated terms are averaged over a rotor period showing the power of the MAS technique. The downside to this averaging is that direct structural knowledge is lost. However, by reintroducing the dipole-dipole coupling interactions under MAS experiments, internuclear distances can be determined, since the dipolar coupling scales as one over the internuclear distance cubed, cf. the definition of $b_{I S}$. An example of such an experiment is the back-to-back $(\mathrm{BaBa})$ [39-41] sequence which probes spin-pair connectivities observed indirectly via double-quantum (DQ) coherences. The BaBa technique will be discussed in Section 3.3.

In a multi-spin system, homonuclear dipole-dipole coupling interactions that share one common spin do not commute. This makes it extremely hard to gain insight into the weaker couplings since the larger dipolar interactions average the weaker ones - a phenomenon known as dipolar truncation [42]. The non-commuting interactions make, in principle, a multi-spin approach inevitable. But since the higher order terms in the Magnus expansion get less important at higher MAS frequencies, the use of faster MAS probes render the two-spin correlations more predominant [43]. As a rule of thumb, MAS frequencies should be used at or beyond the dipolar strength of the most proximate nuclei [43]. For example, the dipolar strength of two static ${ }^{1} \mathrm{H}$ nuclei separated in space by $1.8 \AA$ is ca. $-20.6 \mathrm{kHz}$, while that of two ${ }^{13} \mathrm{C}$ nuclei separated by $1.5 \AA$ is on the order of $-2 \mathrm{kHz}$.

Heteronuclear spins have appreciably different resonance frequencies. For example, on a 9.4 T spectrometer, ${ }^{1} \mathrm{H}$ spins resonate at ca. $400 \mathrm{MHz}$ and ${ }^{13} \mathrm{C}$ spins resonate at ca. 100 $\mathrm{MHz}$, a difference of $300 \mathrm{MHz}$. A large chemical shift difference of tens or hundreds of 
$\mathrm{MHz}$ will truncate the planer term, also known as the zero-quantum term, in the dipoledipole coupling interaction [31]. While still addressing spin $I$ and spin $S$ but now in a heteronuclear two-spin system, Eq. (0.12) simplifies to

$$
H_{I S}(t)=\omega_{I S}(t) 2 I_{z} S_{z} .
$$

Only the longitudinal spin term is present in Eq. (0.14) and all heteronuclear dipole-dipole coupling interactions commute in a multi-spin system making the analysis much more straightforward. One of the best known pulse sequences in solid-state NMR spectroscopy, the rotational-echo double-resonance NMR (REDOR) experiment [44], employs appropriately placed $\pi$ pulses to avoid averaging of the heteronuclear dipolar coupling interactions by MAS. REDOR has been used in numerous cases to extract precise dipolar couplings and the inventor, Prof. Schaefer, was commemorated recently for his contributions to solid-state NMR spectroscopy [45].

The isotropic J-coupling interaction between spin $I$ and spin $S$ can be described as

$$
H_{J}=2 \pi J_{I S} \mathbf{I} \cdot \mathbf{S}=2 \pi J_{I S}\left(I_{x} S_{x}+I_{y} S_{y}+I_{z} S_{z}\right) .
$$

The size of this interaction is often in the $10-200 \mathrm{~Hz}$ range. If the difference in isotropic chemical shift between spins $I$ and $S$ is larger than the size of the J-coupling, then the planer terms $I_{x} S_{x}+I_{y} S_{y}$ in Eq. (0.15) is truncated, leaving behind only the longitudinal term $I_{z} S_{z}$. 


\section{NMR PULSE SEQUENCES FOR ORGANIC SOLIDS AND SOFT}

\section{MATERIALS}

In material science, the characterization of molecular structure is of utmost importance. Focusing on, say, $\pi$-conjugated polymers, which consist of a backbone with alternating single and double bonds, it proves valuable to know the ratio of $\mathrm{sp}^{2}$-hybridized ${ }^{13} \mathrm{C}$ atoms relative to $\mathrm{sp}^{3}$-hybridized ${ }^{13} \mathrm{C}$ atoms and the ratio of potential functional groups such as esters and carboxyl acids. However, structural insight at the molecular level is highly desired but very challenging to obtain in non-crystalline materials. In this contribution, we will mostly focus on the NMR active nuclei ${ }^{1} \mathrm{H}$ and ${ }^{13} \mathrm{C}$, which posses a natural abundance close to $100 \%$ and $1.1 \%$, respectively. Other important NMR nuclei in the field of material science are ${ }^{14} \mathrm{~N},{ }^{19} \mathrm{~F},{ }^{27} \mathrm{Al}$, and ${ }^{31} \mathrm{P}$ to highlight a few.

In the following section, only a selected subset of the available solid-state NMR experiments used for elucidating quantitative and structural information on soft organic nanomaterials will be highlighted. Readers are encouraged to supplement the following discussion with other excellent reviews by experts in the field, such as Brown [46, 47], Levitt [32] and Saalwächter [48]. Apart from the selected experiments and applications in this section, solid-state NMR spectroscopy is also capable of (i) spectral editing, e.g. distinguishing $\mathrm{C}, \mathrm{CH}, \mathrm{CH}_{2}$ and $\mathrm{CH}_{3}$ groups [49-51], (ii) finding diffusion coefficients [52], (iii) estimating domain sizes, e.g. by observing spin diffusion [53-56], and (iv) probing local dynamics [38, 57-59]. Most of the experiments presented in this contribution are developed for high-field NMR spectrometers. Low-field NMR spectroscopy is a distinct research field, 
which can be applied fruitfully on soft matter systems as recently reviewed by Schäler et al. [60].

\subsection{Quantitative NMR (qNMR)}

NMR spectroscopy has the capacity to provide quantitative insight into questions related to molecular structure, such as the ratios among different chemical groups in a molecule. The deployment of NMR for this purpose is known as quantitative NMR (qNMR) [61, 62]. In liquid-state NMR spectroscopy, Malz and Jancke have established a validated protocol for qNMR [63]. To our knowledge, such a protocol has not yet been established within solidstate NMR spectroscopy. In its standard implementation for solid samples, the qNMR experiment makes use of the MAS technique in order to observe only the isotropic signal. A short excitation pulse makes the spins amenable for observation and the resonance frequencies are encoded in the free induction decay (FID). If necessary, heteronuclear decoupling is applied during acquisition. Examples of heteronuclear decoupling schemes are SPINAL-64 [64], XiX [65], SW $\mathrm{f}_{\mathrm{f}}$-TPPM [66], UTPD [67] and rCW [68], which are often used when observing ${ }^{13} \mathrm{C}$ spins surrounded by ${ }^{1} \mathrm{H}$ spins. To increase the signal-to-noise ratio in the resulting spectrum obtained by a discrete Fourier transform of the FID, several scans are co-added. The optimal delay between subsequent scans depends on the excitation flip angle [69]. If $\pi / 2$ pulses are used, it is advisable to set the recycle delay such that it is longer than five times the longitudinal relaxation time $\left(T_{1}\right)$ to ensure (almost) full Boltzmann equilibrium with respect to the large static magnetic field. The resulting resolved spectrum contains signals that can be integrated to yield quantitative relative ratios. The 
spectrum can also be used for assignment based directly on the chemical shift and the intensities.

\subsection{Background and ringing suppression}

The above experimental method is referred to as a direct polarization (DP) or a singlepulse (SP) experiment, cf. Figure 1a for schematic representation. In reality, several issues can make it difficult to extract quantitative information from these experiments. Such issues include strong background signals from the NMR probe/rotor that overlap with the signals originating in the sample, and acoustic ringing and transient signals in the probe after rf pulsing. Materials extraneous to the coil, such as endcaps, inserts and rotors might contain NMR active nuclei, which could contribute unwanted signals to the spectrum. Acoustic ringing comes about as the metal parts start to vibrate as a consequence of rf pulsing. These vibrations can potentially give rise to signals during acquisition since they might last up to tens or hundreds of $\mu$ s. A dead time period after rf pulsing and prior to acquisition ensures (sufficient) ringdown of the electronics. However, a temporal shift (i.e. waiting until dead time has passed instead of immediate acquisition) imparts a linear phase shift across the detected frequency range. These issues usually manifest in the spectrum as baseline and phase distortions. Several methods [70-78] have been developed to alleviate some of these problems. We will briefly discuss a few of these techniques now.

The use of a Hahn echo, which typically extends over two rotor periods with a $\pi$ pulse in the middle, reduces probe background, ringing and dead time effects. A schematic representation is seen in Figure $1 \mathrm{~b}$. The period from the end of the refocusing pulse to the 
beginning of acquisition at the top of the signal echo is often considerably longer than the dead time of the receiver and might be longer than the acoustic ringdown period. Spins in the probe far from the center of the rf coil will not be refocused by the pulse and should therefore not give rise to (large) signals. In general, however, an echo sequence cannot address all of the concerns related to the deployment of qNMR [75].

Chen et al. [73] proposed a method consisting of combining the spectra of two DP experiments to suppress background signals, cf. Figure 1c. In the most common form, a $\pi / 2$ excitation pulse and a $\pi$ pulse is applied, respectively. Assuming linear excitation of the spins in the probe far from the if coil, the background-reduced spectrum appears by scaling the " $\pi$ "spectrum by a half and then subtracting it from the " $\pi / 2$ "spectrum. A drawback of this approach is that two experiments have to be performed, both with repetition delays, effectively doubling the experimental time.

A technique which does not involve the doubling of the repetition delays is the elimination of artifacts in NMR spectroscopy EASY approach [75, 76]. The EASY procedure is displayed in Figure 1d. The general idea is to acquire two DP experiments with $\pi / 2$ excitation pulses immediately after one another. That is, when the FID of the first experiment has decayed, which happens within a time period of the transverse relaxation time $\left(T_{2}\right)$, the same experiment is initiated and the second FID is recorded. As long as $T_{2}$ of the sample is much shorter than $T_{1}$, very little magnetization originating from the sample is present in the second FID. On the contrary, the background signals that fall into the small flip angle regime are present. A background reduced FID appears by subtracting the second FID from the first. An additional advantage of the EASY setup is that ringdown effects are likewise cancelled in the subtraction step as these effects follow directly the if 
pulse and are, therefore, the same in the two acquisition periods. Instead of direct subtraction, receiver phase cycling can also be used to achieve the differential FID. For long observation times on, say, ${ }^{13} \mathrm{C}$ nuclei, the implementation of two subsequent acquisition periods with decoupling can put some strain on the duty cycle of the hardware. Jaeger and Hemmann $[75,76]$ claim that the second acquisition time can be shortened to much less than $T_{2}$ of the sample as the broad ringing/background signals are contained in the first few data points.

As a final remark on the suppression of background signals, we note that in some cases, unwanted signals simply have to be identified from an empty rotor. Caution must be exercised while doing so since matching and tuning might potentially change when moving from a full rotor to an empty one.
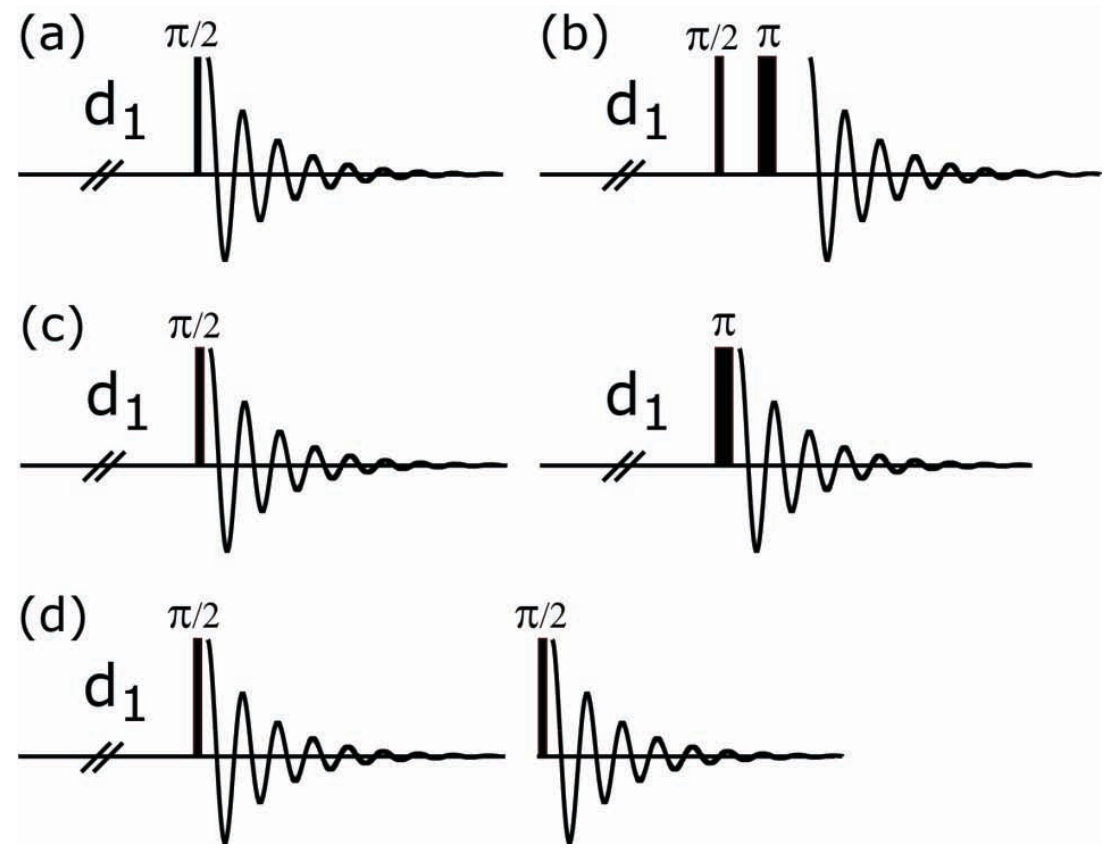

Figure 1. Schematic representations of (a) direct polarization, (b) Hahn echo, (c) scheme by Chen et al. and (d) EASY experiments. $d_{1}$ refers to repetition delays and short filled pulses signify $\pi / 2$ rotations, longer filled pulses signify $\pi$ rotations. For phase cycling scheme, please refer to original articles. 


\subsection{Double-quantum single-quantum spectroscopy}

Beyond quantification of moieties, an equally important aspect of NMR spectroscopy is to probe structural information in the form of spin-pair connectivity. If the sample is amorphous or crystalline/amorphous, NMR spectroscopy is one of the preferred techniques to obtain local structural information on the atomic level.

Dipolar interactions can, by virtue of the dependency on the internuclear distance $\left(1 / r^{3}\right.$ cf. Eq. (0.13)), be used to probe spin-pair connectivity. We will briefly comment on the double-quantum (DQ) experiment utilizing the Back-to-Back (BaBa) sequence [39-41] to find distance constraints among same spin species. Reviews on DQ NMR can be found in Refs. $[48,79]$. The basic building block of the BaBa sequence and the schematic pulse sequence can be seen in Figure $2 \mathrm{a}$ and Figure $2 \mathrm{~d}$, respectively. In the toggling frame, where the Hamiltonian is toggled to another frame by an if pulse, the dipolar coupling interactions are not averaged by MAS and a first-order analysis gives an effective DQ Hamiltonian

$$
H_{e f f}^{(1)}=H_{D Q}=\omega_{e f f}\left(\begin{array}{ll}
H_{x} S_{x} & I_{y} S_{y}
\end{array}\right)
$$

Spin $I$ and spin $S$ _refer to a homonuclear spin-pair, e.g. a ${ }^{1} \mathrm{H}-{ }^{1} \mathrm{H}$ pair. The expression of the effective coupling strength can be found in Ref. [80]. Using the fictitious DQ spin-1/2 operators, $I_{x}^{D Q}=I_{x} S_{x}-I_{y} S_{y}, I_{y}^{D Q}=I_{y} S_{x}+I_{x} S_{y}, I_{z}^{D Q}=\frac{1}{2}\left(I_{z}+S_{z}\right)$, the initial (DQ) polarization will evolve into

$$
\begin{aligned}
\rho\left(t_{r}\right) & =U\left(t_{r}\right)\left(I_{z}+S_{z}\right) U\left(t_{r}\right)^{\dagger}=e^{-i \omega_{e f t} t_{r} I_{x}^{D Q}} 2 I_{z}^{D Q} e^{i \omega_{e f t} t_{r} I_{x}^{D Q}} \\
& =2\left(I_{z}^{D Q} \cos \left(\omega_{e f f} t_{r}\right)-I_{y}^{D Q} \sin \left(\omega_{e f f} t_{r}\right)\right)
\end{aligned}
$$


The DQ term, $I_{y}^{D Q}$, encodes the sum of the chemical shifts in the indirect dimension. The dwell time can be chosen as a rotor period such that $t_{1}=n t_{r}$.

$$
\begin{aligned}
\rho\left(t_{r}+t_{1}\right) & =U_{\Sigma}\left(n t_{r}\right)\left(2\left(I_{z}^{D Q} \cos \left(\omega_{e f f} t_{r}\right)-I_{y}^{D Q} \sin \left(\omega_{e f f} t_{r}\right)\right)\right) U_{\Sigma}\left(n t_{r}\right)^{\dagger}, \quad U_{\Sigma}\left(n t_{r}\right)=e^{-i \omega_{\Sigma} n t_{r} I_{z}^{D Q}} \\
& =2\left(I_{z}^{D Q} \cos \left(\omega_{e f f} t_{r}\right)-I_{y}^{D Q} \cos \left(\omega_{\Sigma} n t_{r}\right) \sin \left(\omega_{e f f} t_{r}\right)+I_{x}^{D Q} \sin \left(\omega_{\Sigma} n t_{r}\right) \sin \left(\omega_{e f f} t_{r}\right)\right)
\end{aligned}
$$

$I_{y}^{D Q}$ will be reverted back into polarization by a reconversion block (similar to the transformation in Eq. (0.17) but using an opposite rotation).

$$
\begin{aligned}
\rho\left(t_{r}+t_{1}+t_{r}\right)_{I_{z}^{D Q}} & =U\left(t_{r}\right) \rho\left(t_{r}+t_{1}+t_{r}\right) U\left(t_{r}\right)_{I_{z}^{D Q}}^{\dagger} \\
& =2\left(I_{z}^{D Q} \cos ^{2}\left(\omega_{e f f} t_{r}\right)+I_{z}^{D Q} \sin ^{2}\left(\omega_{e f f} t_{r}\right) \cos \left(\omega_{\Sigma} n t_{r}\right)\right)
\end{aligned}
$$

In Eq. (0.19), only the longitudinal terms are included. Prior to flipping the polarization onto the transverse plane for acquisition, a short z-filter period is applied to remove unwanted terms (like higher order coherences) in the density operator. The duration of the z-filter should be kept short to avoid additional effects from spin-diffusion. A four-step phase cycling with the receiver setting reversed every second step is used to select only the term that encodes the sum of the chemical shifts. This can also be appreciated by realizing that a $\pi / 2$ phase change leads to a sign change in the reconversion block. However, this approach in combination with one in which a constant receiver is set, can be used to obtain a normalization of the intensity $[48,80,81]$.

The important aspect of a $\mathrm{DQ}$ experiment is that the spatial connectivity between two dipolar coupled nuclei appears as a cross-correlation at the sum of the chemical shifts in the indirect dimension. The cross-correlation should ideally be observed on each side of the diagonal with the center being on the diagonal. 
(a) $\mathrm{x}$

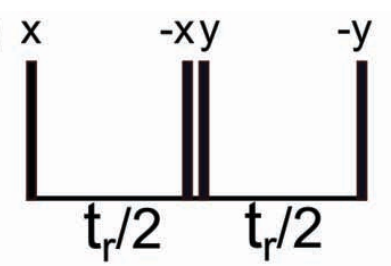

(b)

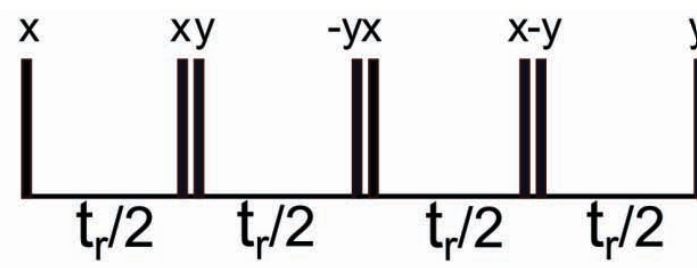

(c)

$=\pi / 2_{\theta}$

(d)

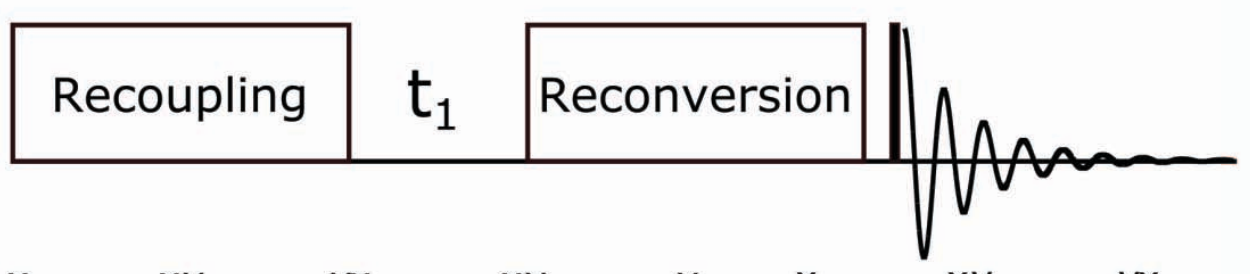

(e)

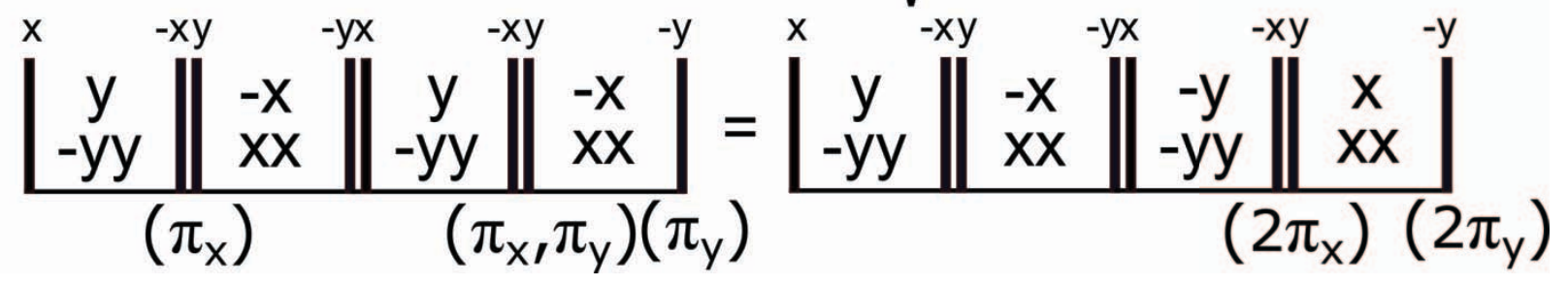

Figure 2. (a) The basic Back-to-Back ( $\mathrm{BaBa}$ ) element and (b) the original broadband version, both applying (c) $\pi / 2$ pulses, can be used in the recoupling and reconversion parts of the DQ-ZQ pulse sequence shown in (d). The broadband version uses, on top of the BaBa element, implicit $\pi$ pulses which refocus the linear parts but leaves the bilinear terms unaffected, as illustrated in (e). The $\pi / 2$ pulses in (e) are only included as a reminder of the toggling frame description, as the sign of the linear terms is to be viewed in that frame. The sign of the bilinear terms reflects the effective DQ Hamiltonian.

In the original $\mathrm{BaBa}$ block element, the linear terms, e.g. the chemical shifts, are not refocused. In Figure $2 b$, the broadband scheme [40] which makes use of implicit $\pi$ pulses is shown. The $\pi$ pulses refocus the linear terms when adding the individual Hamiltonians of more elements, but they do not touch the bilinear terms of the dipole-dipole coupling interactions. This line of reasoning is depicted in Figure 2e. The overall rf field is still cyclic and the $\pi$ pulses are incorporated into the $\pi / 2$ pulses as phase inversions, e.g., $\pi / 2_{\times}$pulse $+\pi_{\mathrm{x}}$ pulse equals $\pi / 2$-x pulse. The scheme of implicit $\pi$ pulses has been extended by Saalwächter et al. [41] to include a xy16 phase-cycle of the $\pi$ pulses in combination with time-reversed $\mathrm{DQ}$ reconversion. The $\mathrm{BaBa}-\mathrm{xy} 16$ sequence has been proven to be 
experimentally robust, which turns out to be useful when, for example, employing very long recoupling and reconversion blocks. In the literature, up to 128 rotor periods dipolar recoupling have been employed [82] using $25.0 \mathrm{kHz}$ MAS. The relaxation-normalized DQ build-up curves described therein obtained as $I_{D Q} /\left(I_{Z Q}+I_{M Q}\right)$ revealed insight into the polymer side-chain dynamics before and after preliminary GPC purification.

In Eq. (0.18), the dwell time in the indirect dimension was set to one rotor period which is sufficient to yield distance constraints. To get more precise distance measurements, it is more convenient to set the dwell time well below a rotor period. In this way, DQ spinning sidebands are generated [83, 84]. The underlying reason is that at a given intermediate time step the average Hamiltonian of the reconversion blocks differs by a phase about the rotor axis compared to the excitation block when using a non rotor synchronized scheme. The mechanism is termed reconversion rotor encoding (RRE). The dipolar sideband patterns originating from multispin effects appear at even orders [85].

The RRE approach has successfully been applied to extract stacking distances in a perylene tetracarboxylydiimide derivative, $\mathrm{C}_{8,7}-\mathrm{PDI}$ [86]. The supramolecular arrangement of these PDI derivatives allows for 1D electronic charge transport, an attractive property for the fabrication of nanoscale devices such as organic solar cells. 

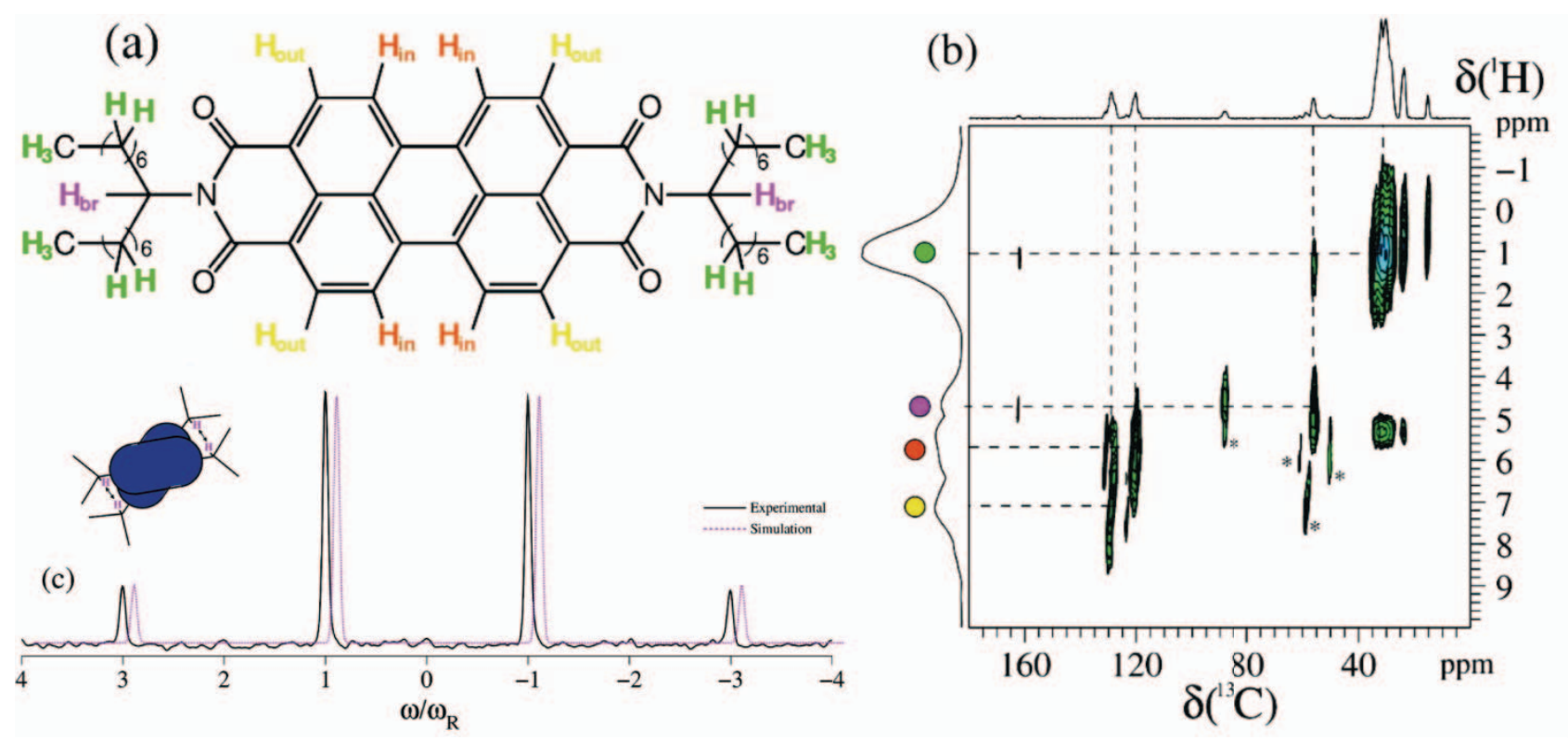

Figure 3. (a) Schematic representation of a $\mathrm{C}_{8,7}-\mathrm{PDI}$ molecule with color coding matching the assignment based on (b) a 2D ${ }^{13} \mathrm{C}\left\{{ }^{1} \mathrm{H}\right\}$ FSLG-HETCOR correlation spectrum using a ramped $\mathrm{CP}$ transfer of $500 \mu \mathrm{s}$. (c) The experimental ${ }^{1} \mathrm{H}_{\mathrm{br}}{ }^{-1} \mathrm{H}_{\mathrm{br}} \mathrm{DQ}$ sideband pattern is extracted from a 2D DQ$S Q{ }^{1} \mathrm{H}^{-1} \mathrm{H}$ correlation spectrum based on 8 rotor periods of $\mathrm{DQ}$ recoupling obtained at $25.0 \mathrm{kHz}$ MAS with the dwell time set to $2.0 \mu \mathrm{s}$ in the indirect dimension. The ${ }^{1} \mathrm{H}_{\mathrm{br}}{ }^{1} \mathrm{H}_{\mathrm{br}} \mathrm{DQ}$ sideband pattern shows significant two-spin correlation, and the ${ }^{1} \mathrm{H}_{\mathrm{br}}-{ }^{1} \mathrm{H}_{\mathrm{br}}$ intermolecular distance is estimated to be $3.7 \pm 0.1 \AA$. All experiments were acquired at $20.0 \mathrm{~T}$. Adapted with permission from Ref. [86]. Copyright 2009 American Chemical Society

Figure $3 a$ shows the schematic representation of a $\mathrm{C}_{8,7}$-PDI molecule. Especially interesting in the context of RRE are the ${ }^{1} \mathrm{H}_{\mathrm{br}}$ nuclei since the intramolecular distance is expected to be ca. $15 \AA$. The ${ }^{1} \mathrm{H}_{\mathrm{br}}$ nuclei have been assigned using a ${ }^{13} \mathrm{C}\left\{{ }^{1} \mathrm{H}\right\}-\mathrm{FSLG}-$ HETCOR experiment with $500 \mu$ s cross-polarization time (vide infra), cf. Figure 3b. In Figure 3c, the ${ }^{1} \mathrm{H}_{\mathrm{br}}-{ }^{1} \mathrm{H}_{\mathrm{br}}$ DQ spinning sideband pattern stemming from the RRE approach using 8 rotor periods of $D Q$ recoupling and an indirect dwell time of $2 \mu$ s indicates an intermolecular distance of $3.7 \pm 0.1 \AA$, revealing an effective ensemble averaged packing distance between successively stacked $\mathrm{C}_{8,7}$-PDI molecules. Also, note that only $\mathrm{DQ}$ 
spinning sidebands of odd orders are present in Figure 3c, emphasizing the dominating two-spin behavior.

The above example shows the power of advanced solid-state ${ }^{1} \mathrm{H}$ MAS NMR spectroscopy to probe local ordering in supramolecular assemblies. In general, NMR spectroscopy is very sensitive to the local electronic environment as manifested via the isotropic chemical shifts. As mentioned in the Introduction, ${ }^{1} \mathrm{H}$ NMR chemical shifts are particularly highly sensitive to phenomenon such as hydrogen bonding and $\pi-\pi$ stacking $[79,87]$.

\subsection{Cross-polarization (CP)}

In the previously described experiments, the focus was placed only a single spin species, e.g. only ${ }^{1} \mathrm{H}$ spins. ${ }^{1} \mathrm{H}$ NMR benefits from a high gyromagnetic ratio, $\sim 100 \%$ natural abundance, and longitudinal relaxation times on the order of (milli)seconds, rendering it possible to have a reasonably fast repetition rate between transients. The disadvantage of ${ }^{1} \mathrm{H}$ solid-state NMR spectroscopy is the limited resonance range of [0-15] ppm and strong dipolar interactions giving rise to very broad signals. In contrast, ${ }^{13} \mathrm{C}$ solid-state NMR spectroscopy, though it suffers from a low natural abundance $(1.1 \%)$ and gyromagnetic ratio (only a quarter relative to ${ }^{1} \mathrm{H}$ ), it posses a reasonably large resonance range of ca. [0200] ppm and longitudinal relaxation times on the order of tens of seconds.

The cross-polarization (CP) experiment $[88,89]$, shown schematically in Figure $4 \mathrm{a}$, is probably the most well-known experiment in solid-state NMR spectroscopy. This 
experiment takes advantage of the above-mentioned facts to enhance the magnetization of the ${ }^{13} \mathrm{C}$ spins up to $\gamma_{{ }_{H}} / \gamma_{{ }^{3} \mathrm{C}}$ times the magnitude of the equilibrium Boltzmann polarization and exploit the much shorter longitudinal relaxation times of the protons compared to those of carbons. CP can be understood from the perspective of spin thermodynamics, which has been addressed by numerous books and articles Refs. [27, 90]. It can also be described from an average Hamiltonian analysis, which can shed light on the coherent evolution of a spin-pair system at a much more detailed level. Both of these analysis frameworks will be presented in the following few paragraphs.

(a) $90^{\circ}$

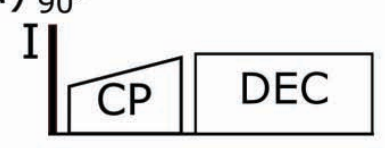

$\mathrm{S}$

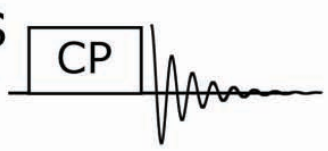

(c)
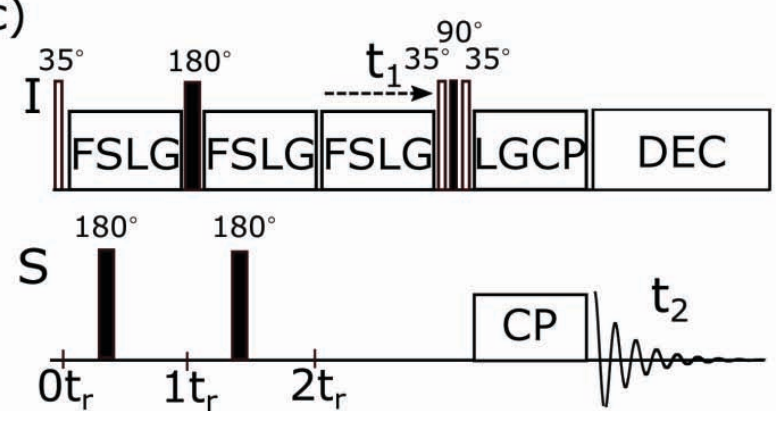

(b) 50

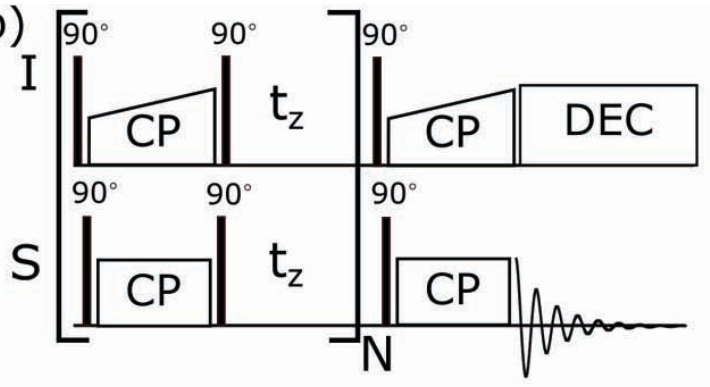

(d)

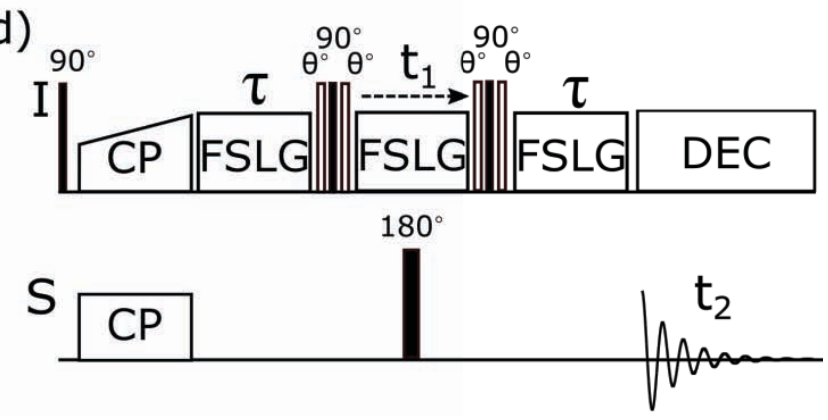

Figure 4. (a) The standard $S\{1\} C P / M A S$ NMR experiment, (b) the multiCP experiment, (c) the MELODI-HETCOR NMR experiment and (d) the MAS-J-HMQC NMR experiment with $\Theta$ denoting the magic angle, ca. 54.7 degrees. In general, different time periods are not drawn to scale. For phase cycling schemes we refer to original articles.

The CP experiment is initiated via the excitation of the $I$ spins, say protons. The magnetization is then transferred to the $S$ spins, say carbons, prior to detection. The 
entire block can be repeated whenever the polarization of the $I$ spins has reached the wanted magnitude, within hardware limitations.

To grasp the strength and potential pitfalls of $\mathrm{CP}$, details about the magnetization transfer need to be appreciated. Immediately after excitation, the magnitude of the magnetization along the $x$ axis is proportional to the Boltzmann inverse spin temperature, $\beta_{I}^{0}$, as described by Eq. (0.10). Subsequently, spin lock fields consisting of $x$ phase rf fields are applied on both channels and the rf fields are adjusted according to the Hartmann-Hahn $(\mathrm{HH})$ matching condition [91]. In static experiments, the $\mathrm{HH}$ condition is to match the two rf fields; in MAS experiments, the difference in the rf field amplitudes is set to one or two times the spinning frequency. In practice, a ramp of the rf amplitude on one of the channels is often used to make the experiment more robust [92].

In the spin-lock frame, the size of the $I$ magnetization is far larger than justified by the rf field. Therefore, the $I$ spins are said to have a low spin temperature or high inverse spin temperature, $\beta_{I}$. On the other hand, at the beginning there is no $S$ magnetization in the transverse plane, which corresponds to an infinitely high temperature or zero inverse temperature, $\beta_{S}$. Before proceeding, recall that the $x$ magnetization of the $S$ spins can be calculated as

$$
M_{s}=\gamma_{s} \operatorname{Tr}\left[\rho S_{x}\right], \quad \rho ; Z^{-1}\left(1-\beta_{S} \omega_{1, S} S_{x}\right)
$$

We can therefore continue the description by referring to the inverse temperatures.

The trick in spin thermodynamic is to view the system consisting of a very large reservoir of $I$ spins that is connected to the reservoir of $S$ spins via dipole-dipole coupling 
interactions. The $\mathrm{HH}$ match allows energy to flow on the time scale of the $\mathrm{CP}$ time constant, $T_{I S}$, and, eventually, the inverse spin temperatures will equilibrate. However, both the $I$ spins and the $S$ spins are connected to the surroundings as well. This will give rise to relaxation processes described by the $T_{1, \rho}^{I}$ and $T_{1, \rho}^{S}$ spin-lattice relaxation times in the rotation frames as, eventually, the Zeeman equilibrium will prevail and no magnetization will be present in the transverse plane. These can be taken into account as coupled differential equations, and the magnetization of the $S$ spins can be found as a function of time.

Assuming a good $\mathrm{HH}$ match, very long relaxation times and that the protons outnumber the carbons $\left({ }^{13} \mathrm{C}\right)$, i.e. $N_{S} / N_{I} \approx 0$, the magnitude of the $S$ spins magnetization is described as

$$
S(t)=\frac{\gamma_{I}}{\gamma_{S}} S_{e q}\left(1-e^{-t / T_{I S}}\right)
$$

with $S_{e q}$ denoting the size of the Zeeman equilibrium magnetization. According to Eq. (0.21), the magnetization of the $S$ spins is enhanced by $\gamma_{I} / \gamma_{S}$, which amounts to a factor of four in the case of protons and carbons. Also, the intensity buildup of CP depends on $T_{I S}$, which is heavily dependent on the dipolar coupling of the spins. If the dipolar coupling is large, $T_{I S}$ is small and hence, a good enhancement is obtained quickly. On the other hand, if dipolar coupling is (very) small, it will require longer times, if ever, before benefits of CP is apparent. This is very useful as it allows us, for instance, to distinguish mobile parts from rigid parts, and strongly coupled parts from those that are weakly coupled [9395] [96]. 
Though Eq. (0.21) suggests a $\gamma_{I} / \gamma_{S}$ gain, the maximum is seldom observed. When relaxation of the $I$ spins during the CP step is included, the magnetization of the $S$ spins becomes

$$
S(t)=\frac{\gamma_{I}}{\gamma_{S}} S_{e q}\left(1-T_{I S} / T_{1, \rho}^{I}\right)^{-1}\left(e^{-t / T_{1, \rho}^{I}}-e^{-t / T_{I S}}\right) .
$$

The exponential decaying term induced by the relaxation of the $I$ spins means that the signal enhancement of the $S$ spins is ultimately limited by $T_{1, \rho}^{I}$.

Finally, the spin thermodynamics picture assumes that the inverse temperature in the abundant spin bath is the same at any given point in time. That assumption is valid as long as these spins are strongly connected, i.e. as long as spin diffusion happens sufficiently fast. For protons, that is often the case at low/intermediate MAS frequencies.

Before continuing on to other NMR experiments, we would like to stress the power of the simple CP $\left({ }^{13} \mathrm{C}\right)$ and DP $\left({ }^{1} \mathrm{H}\right)$ approach via the examination of a rod-coil block copolymer system [97]. Rod-coil block copolymers constitute an interesting diblock copolymer class as various interactions between segments define phase separation and liquid crystallinity [98-100], and the capacity to self-assemble makes these systems interesting to the field of organic photovoltaics [101]. The aim of the study in Ref. [97] was to shed light on the non-covalent interactions in oligo( $p$-benzamide)-poly(ethylene glycol) $\left(\mathrm{OPBA}_{(\text {rod })}-\mathrm{PEG}_{(\text {(coil) }}\right)$ copolymers to elucidate the driving forces governing the aggregation behavior. 

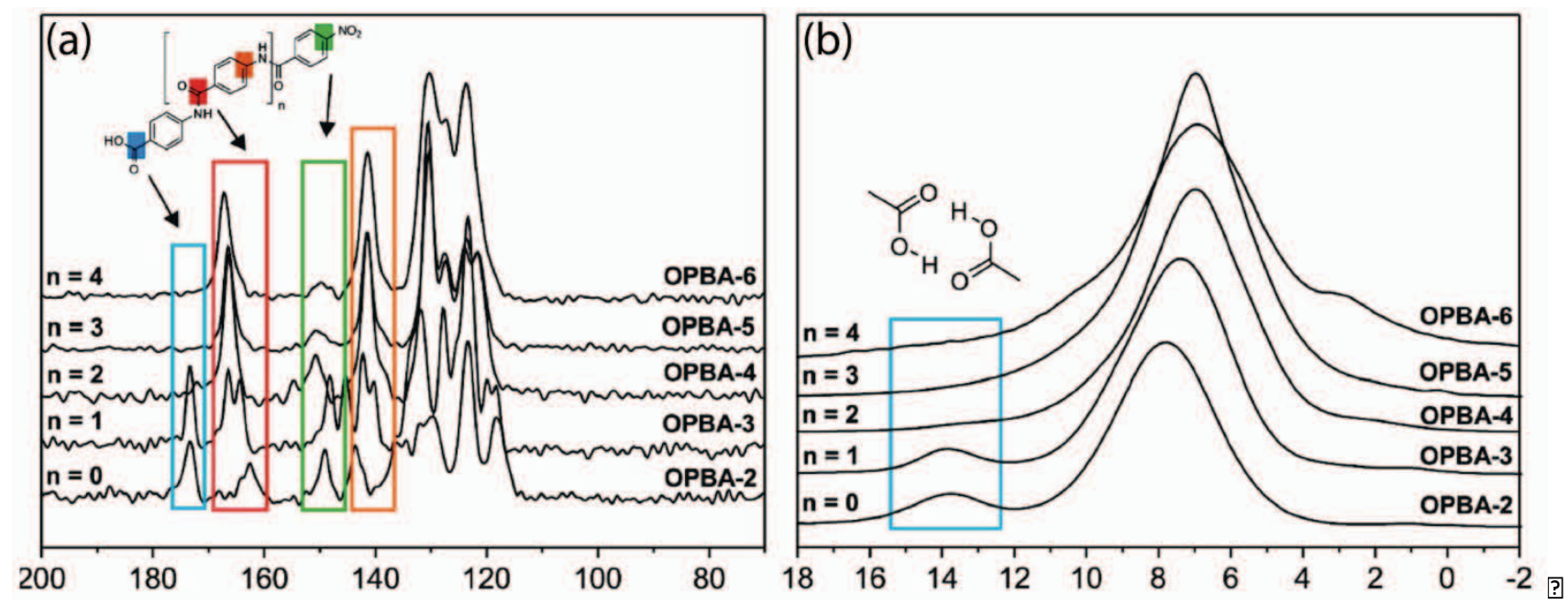

Figure 5. (a) A series of ${ }^{13} \mathrm{C}\left\{{ }^{1} \mathrm{H}\right\} \mathrm{CP} / \mathrm{MAS}$ spectra acquired at $11.7 \mathrm{~T}$ using $25 \mathrm{kHz}$ MAS and $3 \mathrm{~ms}$ $\mathrm{CP}$ contact time and (b) a series of ${ }^{1} \mathrm{H}$ MAS NMR spectra acquired at $16.4 \mathrm{~T}$ using $30 \mathrm{kHz} \mathrm{MAS}$ on OPBA polymers of increasing number of repeat units. We see a significant change in both series when going from OPBA-3 to OPBA-4. The change indicates a shift in the hydrogen bonding mechanism from one that is dominated by carboxylic acid hydrogen bonding to that which is dominated by amide hydrogen bonds. Adapted with permission from Ref. [97]. Copyright 2010 American Chemical Society.

The ${ }^{13} \mathrm{C}\left\{{ }^{1} \mathrm{H}\right\}$ CP/MAS spectra in Figure 5 a clearly show a change in the resonance frequencies when going from OPBA-3 to OPBA-4. Most striking is the shift of the $172 \mathrm{ppm}$ signal to $\sim 164 \mathrm{ppm}$. The former resonance frequency is associated with $\mathrm{COOH}$ groups participating in hydrogen bonding constellations. In the ${ }^{1} \mathrm{H}$ MAS NMR spectra in Figure $5 \mathrm{~b}$, we likewise see a significant change when going from OPBA-3 to OPBA-4. The signal at ca. $14 \mathrm{ppm}$ which is ascribed to the protons taking part in the hydrogen bonds among carboxylic acids is less pronounced for longer polymers. As a side-remark, please note the hydrogen-bonded ${ }^{1} \mathrm{H}$ nuclei often resonate in the $8-20 \mathrm{ppm}$ range [17]. The obvious conclusion for the observations in Figure 5 is that for the short polymers, the packing is dominated by the hydrogen bonding of two carboxylic acid groups, while for the longer polymers, the increasing number of the much weaker amide hydrogen bonds overcomes 
the earlier mechanism, resulting in a different molecular packing. In addition, the resolution of the ${ }^{13} \mathrm{C}\left\{{ }^{1} \mathrm{H}\right\}$ CP/MAS spectra gets worse for longer polymers. This is ascribed to a decreasing crystallinity of the samples. In general, a very homogeneous sample gives rise to narrow signals while the signals will be broadened for a more inhomogeneous sample.

In a heteronuclear two-spin system, spin $I$ and spin $S$ an AHT analysis to the firstorder reveals the coherent $\mathrm{CP}$ transfer between the spins. We assume the difference in rffield strengths equals the MAS frequency, e.g. by setting the rf field on the $I$ channel to four times the MAS frequency and the rf field on the $S$ channel to three times the MAS frequency. Transforming the description into the rf field frames and averaging over one rotor period provides us with an effective Hamiltonian described by

$$
\begin{aligned}
H_{I S, e f f}^{(1)} & =\omega_{e f f}\left(\beta_{P R}\right)\left(\left(I_{z} S_{z}+I_{y} S_{y}\right) \cos \gamma_{P R}+\left(I_{z} S_{y}-I_{y} S_{z}\right) \sin \gamma_{P R}\right) \\
& =\omega_{e f f}\left(\beta_{P R}\right)\left(Z^{-} \cos \gamma_{P R}+Y^{-} \sin \gamma_{P R}\right)=\omega_{e f f}\left(\beta_{P R}\right) e^{i \gamma_{P R} X^{-}} Z^{-} e^{-i \gamma_{P R} X^{-}}
\end{aligned}
$$

Eq. (0.23) is rewritten in terms of generalized fictitious $Z Q$ spin-1/2 operators, $X^{-}=\left(I_{x}-S_{x}\right) / 2, \quad Y^{-}=\left(I_{z} S_{y}-I_{y} S_{z}\right)$ and $Z^{-}=\left(I_{z} S_{z}+I_{y} S_{y}\right)$, to emphasize the relevant rotation subspace. Please note that the effective dipolar coupling only depends on the $\beta_{P R}$ Euler angle as $\gamma_{P R}$ appears as a phase. Using the effective Hamiltonian in Eq. (0.23), the initial magnetization on spin $I$ is transferred to spin $S$ as

$$
\left.U_{I S}(t) I_{x} U_{I S}^{\dagger}(t)\right|_{S_{x}}=\sin ^{2}\left(\frac{\omega_{e f f}\left(\beta_{P R}\right)}{2} t\right) S_{x}, \quad I_{x}=\frac{1}{2}\left(I_{x}+S_{x}\right)+\frac{1}{2}\left(\begin{array}{ll}
I_{x} & S_{x}
\end{array}\right)=X^{+}+X^{-} .
$$

In principle, the duration of transfer should be multiple rotor periods to make AHT valid. We have also, in Eq. (0.24), highlighted a trick to perform a calculation in the fictitious ZQ 
space. The transfer is characterized by not having any dependency on the $\gamma_{P R}$ angle which enables a transfer efficiency of up to ca. $73 \%$ of the initial magnetization of spin $I$. This phenomenon is often referred to as $\gamma$-encoding [102].

For very fast MAS frequencies ( $>50 \mathrm{kHz}$ ), it can also prove efficient to match the rf fields such that the sum equals the MAS frequency $[103,104]$. This will lead to an effective DQ Hamiltonian which is fully able to mediate transfer of magnetization. A change in sign of the transferred magnetization is characteristic of this DQ transfer [105].

\subsection{Lee-Goldburg and cross-polarization (LGCP)}

Another version of $\mathrm{CP}$ uses homonuclear decoupling during $\mathrm{CP}$ to make the spin response more pair-wise. The Lee-Goldburg CP (LGCP) $[106,107]$ applies if irradiation along with a specific carrier offset (LG decoupling) [108] to reduce the strong dipolar couplings among, say, protons while still transferring magnetization under $\mathrm{HH}$ (sideband) match conditions. It proves instructive to go into detail of the LG mechanism as this sequence has inspired many of the later proposed homonuclear decoupling techniques such as frequency-switch LG (FSLG) [109, 110] and phase-modulated LG (PMLG) [111]. To better appreciate the idea behind LG, we will in the following restrict our discussion to the static case. In addition, we will partially make use of the irreducible spherical tensor operators as these have convenient transformation properties under rotation [27]. The Hamiltonian for offresonance if irradiation and a homonuclear dipolar coupled $I, S$ spin-pair in the rotating frame is assumed to be 


$$
H=\omega_{o f f} J_{z}+\omega_{r f} J_{x}+\omega_{I S}\left(2 I_{z} S_{z}-I_{x} S_{x}-I_{y} S_{y}\right) \neq \omega_{o f f} J_{z}+\omega_{r f} J_{x} \quad \omega_{I S} \sqrt{6} T_{2,0}^{I S},
$$

with $J=I+S$. The first two terms can be written as

$$
\omega_{o f f} J_{z}+\omega_{r f} J_{x}=\omega_{r f, e f f}\left(J_{z} \cos \theta+J_{x} \sin \theta\right)=\omega_{r f, e f f} e^{-i \theta J_{y}} J_{z} e^{i \theta J_{y}}
$$

Tilting the description about the $y$ axis by $-\theta$ makes the effective rf field point along the $z$ axis in the new frame. A subsequent transformation into the interaction of the effective rf field modulates the dipole-dipole coupling interaction

$$
H^{0 / 0}=\omega_{I S} \sqrt{6} \sum_{m=-2}^{2} T_{2, m}^{I S} d_{m, 0}^{(2)}(-\theta) e^{i m \omega_{r, e f f} t}
$$

Here, we have used the general rotation properties of the irreducible spherical tensors

$$
T_{j, m}^{F 2}=R\left(\Omega_{F 1 F 2}\right) T_{j, m}^{F 1} R^{-1}\left(\Omega_{F 1 F 2}\right) \sum_{m^{\prime}=-2}^{2} T_{j, m^{\prime}}^{F 1} D_{m^{\prime}, m}^{(j)}\left(\Omega_{F 1 F 2}\right), \quad D_{m^{\prime}, m}^{(j)}\left(\Omega_{F 1 F 2}\right)=e^{-i m^{\prime} \alpha} d_{m^{\prime}, m}^{(j)}(\beta) e^{-i m \gamma},(0.2
$$

with $\Omega_{F 1 F 2}$ denoting the set of Euler angles relating the two frames. Over a $2 \pi / \omega_{r f, \text { eff }}$ period, all oscillating terms in Eq. (0.27) will be averaged and only the $m=0$ term will survive. However, recalling the "magic" of MAS, i.e. $d_{0,0}^{(2)}(-\theta)=\left(3 \cos ^{2}(-\theta)-1\right) / 2$, we can set $\theta$ equal to 54.7 degrees thereby effectively suppressing the homonuclear dipole-dipole coupling interaction. By construction, the effective field in the rotating frame is inclined at 54.7 degrees with respect to the static magnetic field, and the strength of the effective field in LG is 1.22 times larger than the applied rf field. As mentioned above, LG irradiation makes $\mathrm{CP}$ transfer between a ${ }^{1} \mathrm{H},{ }^{13} \mathrm{C}$ spin-pair less susceptible to influence from the 
surrounding protons. Hence, the major difference between CP and LGCP is the suppression of spin diffusion in the latter.

\subsection{Comparison of DP, CP and INEPT}

To identify rigid and mobile molecular segments, Nowacka et al. [93-95] developed a theoretical model to estimate the CP and INEPT intensities as a function of the rotational correlation time, $\tau_{c}$, and $\mathrm{C}-\mathrm{H}$ bond order parameter, $S_{\mathrm{CH}}$, in MAS experiments. In addition to the CP signal according to Eq. (0.22), the signal ${ }^{13} \mathrm{C}$ intensity of the INEPT $[96,112]$ experiment can be determined as

$$
S(t)=\frac{\gamma_{I}}{\gamma_{S}} S_{e q} n \sin \left(2 \pi J_{I S} \tau_{1}\right) \sin \left(2 \pi J_{I S} \tau_{2}\right) \cos ^{n-1}\left(2 \pi J_{I S} \tau_{2}\right)\left(e^{-2 \tau_{1} / \tau_{2}^{I}-2 \tau_{2} / T_{2}^{S}}\right)
$$

Here, $I(S)$ denotes a ${ }^{1} \mathrm{H}\left({ }^{13} \mathrm{C}\right)$ spin, $n$ is the number of directly bonded protons, $J_{I S}$ is the scalar coupling, $2 \tau_{1}\left(2 \tau_{2}\right)$ is the time for conversion between in-phase and anti-phase magnetization, and $T_{2}^{I}\left(T_{2}^{S}\right)$ is the transverse relaxation time for spin $I(S)$. If the Boltzmann equilibrium has not been reached, the signal has to be scaled (reduced) appropriately.

To estimate relaxation times and cross polarization times, and thereby signal intensities, Nowacka et al. calculated the reduced spectral density function on the basis of the rotational correlation function in the framework of the random fields model. The reduced spectral density function essentially depends on the correlation time(s) and the order parameter. The theoretical model divides the range of the rotational correlation time, $\tau_{c}$, into four regimes: fast $\left(\tau_{c}<1 \mathrm{~ns}\right)$, fast-intermediate $\left(\tau_{c} \approx 0.1 \mu \mathrm{s}\right)$, intermediate $\left(\tau_{c} \approx 1 \mu \mathrm{s}\right)$, 
and slow $\left(\tau_{c}>0.1 \mathrm{~ms}\right)$. In the fast regime, the CP and INEPT signal intensities do not depend on $\tau_{c}$ but rather depend on the magnitude of the order parameter, $\left|S_{\mathrm{CH}}\right|$. For $\left|S_{\mathrm{CH}}\right|$ $<0.1$, signal enhancement is seen from the INEPT scheme, whereas for larger values of the bond order parameter, CP results in a better signal enhancement. In the intermediate regime, neither CP nor INEPT yields good signals due to fast $T_{1, \rho}$ and $T_{2}$ relaxation. The CP experiment outcompetes INEPT in both the fast-intermediate regime and in the slow regime. Figure 6 summarizes the observations on the CP and INEPT signal intensities as a function of $\tau_{c}$. Note that even though the above case is explicitly described for a methylene $\left(\mathrm{CH}_{2}\right)$ segment, similar results have been obtained for $\mathrm{CH}$ and $\mathrm{CH}_{3}$ segments.

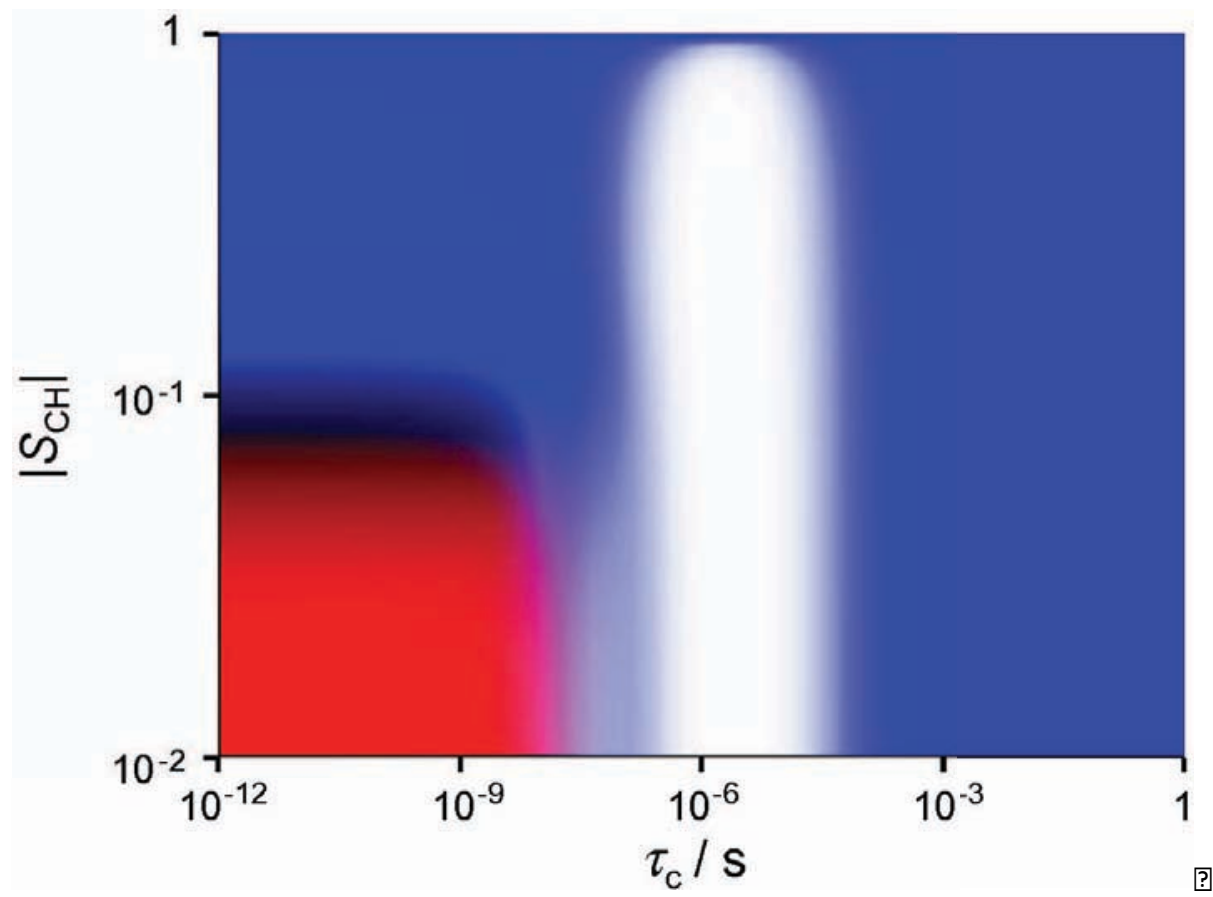

Figure 6. Theoretical ${ }^{1} \mathrm{H}$ to ${ }^{13} \mathrm{C}$ transfer efficiency as a function of the rotational correlation time, $\tau_{c}$ and the magnitude of the $\mathrm{C}-\mathrm{H}$ bond order parameter, $\mathrm{IS}_{\mathrm{CH}} \mathrm{l}$, for a $\mathrm{CH}_{2}$ segment at $11.7 \mathrm{~T}$ and a MAS frequency of $5 \mathrm{kHz}$. The model assumes a mono-exponential correlation function. $\mathrm{CP}$ dominates in the blue area and INEPT dominates in the red area. The white area indicates no signal at all. Adapted with permission from Ref. [93]. Copyright 2013 Elsevier Inc. 
The DP signal (not shown in Figure 6) is quantitative in the range $0.1 \mathrm{~ns}<\tau_{c}<10 \mathrm{~ns}$ and decreases gradually towards zero for longer and shorter correlation times. In the fastintermediate regime, a signal is still obtained, but disappears in the intermediate and slow regimes. By setting the recycle delay $\left(d_{1}\right)$ longer than $5 \mathrm{~s}$. or by including other relaxation mechanisms than pure intramolecular dipolar relaxation, the DP signal could potentially extend further into the intermediate and slow regimes.

\subsection{The multiple cross polarization experiment (multiCP)}

In general, it is difficult to extract quantitative information directly from a CP experiment. To overcome one of the main obstacles in normal CP, the short $T_{1, \rho}^{H}$ of the protons, Johnson and Schmidt-Rohr proposed the multiCP sequence [113]. The sequence can be seen in Figure 4b. It basically contains a series of $\mathrm{CP}$ blocks interleaved with longitudinal relaxation periods of $t_{z}$ duration. Prior to a relaxation period, all magnetization on both the proton and the carbon channels is stored along the $z$ axis by applying $\pi / 2$ pulses.

The basic idea of this approach is simple. The typical CP block transfers magnetization from the protons to the carbons $\left({ }^{13} \mathrm{C}\right)$ as discussed in Section 3.4. However, not all ${ }^{13} \mathrm{C}$ nuclei will have obtained the full enhancement within the duration of a single CP block which is chosen relatively short compared to $T_{1, \rho}^{H}\left(T_{1, \rho}^{H} / t_{C P}>4\right)$. The duration of the relaxation period is chosen such that $t_{z} \approx 2 T_{1, H}$ which should suffice to recover the polarization on the protons, as not much proton magnetization has relaxed during CP (and not that much has been transferred in total as the ${ }^{1} \mathrm{H}$ spins outnumber the ${ }^{13} \mathrm{C}$ spins). The 
polarization on the ${ }^{13} \mathrm{C}$ spins will stay almost constant during $t_{z}$ assuming a long $T_{1, C}$. The $t_{z}$ (or $\sum t_{z}$ ) duration should not be set at too long of a time period as (i) the overall experimental time will increase, and (ii) the enhanced ${ }^{13} \mathrm{C}$ polarization will start to relax towards the normal Boltzmann equilibrium. In the multiCP approach presented in Ref. [113], $14 \mathrm{kHz}$ MAS was applied which, apparently, was sufficient to still have a decent spin diffusion among the protons.

To make the multiCP approach a smooth "drop-in" replacement for conventional CP, the authors summarize a rough guideline. One should, of course, optimize $\pi / 2$ pulses and conventional CP conditions (use the one close to the intensity maximum). In addition, $T_{1, H}$, $T_{1, C}$ and $T_{1, \rho}^{H}$ should be known approximately to be able to set the multiCP parameters correctly. In comparison to DP, the multiCP approach seems very promising as Johnson and Schmidt-Rohr reported more than a 50-fold reduction in measuring time.

Recently, qNMR spectroscopy was used to determine the crystallinity index $(\mathrm{Cl})$ of native cellulose inside the sugarcane bagasse biomass [114]. To get a decent signal-tonoise ratio in reasonable time, Bernardinelli et al. used the multiCP approach. Plant biomass constitutes mainly of cellulose (35-50\%), hemicellulose (20-35\%) and lignin (10$25 \%)$. The idea is to measure the biomass treated with $1 \% \mathrm{H}_{2} \mathrm{SO}_{4}$ that removes practically all of the hemicellulose signals and to measure a sample containing only lignin. This can be accomplished by treating the biomass with $1 \% \mathrm{H}_{2} \mathrm{SO}_{4}$ followed by $0.25 \% \mathrm{NaOH}$. By scaling the second spectrum to match the unique lignin signals in the first, a native cellulose spectrum can be obtained by subtraction. A deconvolution of the signals at 88 ppm and $84 \mathrm{ppm}$ can directly lead to the $\mathrm{Cl}$ determination, as these signals are assigned 
to ${ }^{13} \mathrm{C} 4$ of the crystalline and amorphous cellulose, respectively. The procedure is illustrated in Figure 7 and relies on the quantitative information obtained from NMR spectroscopy.

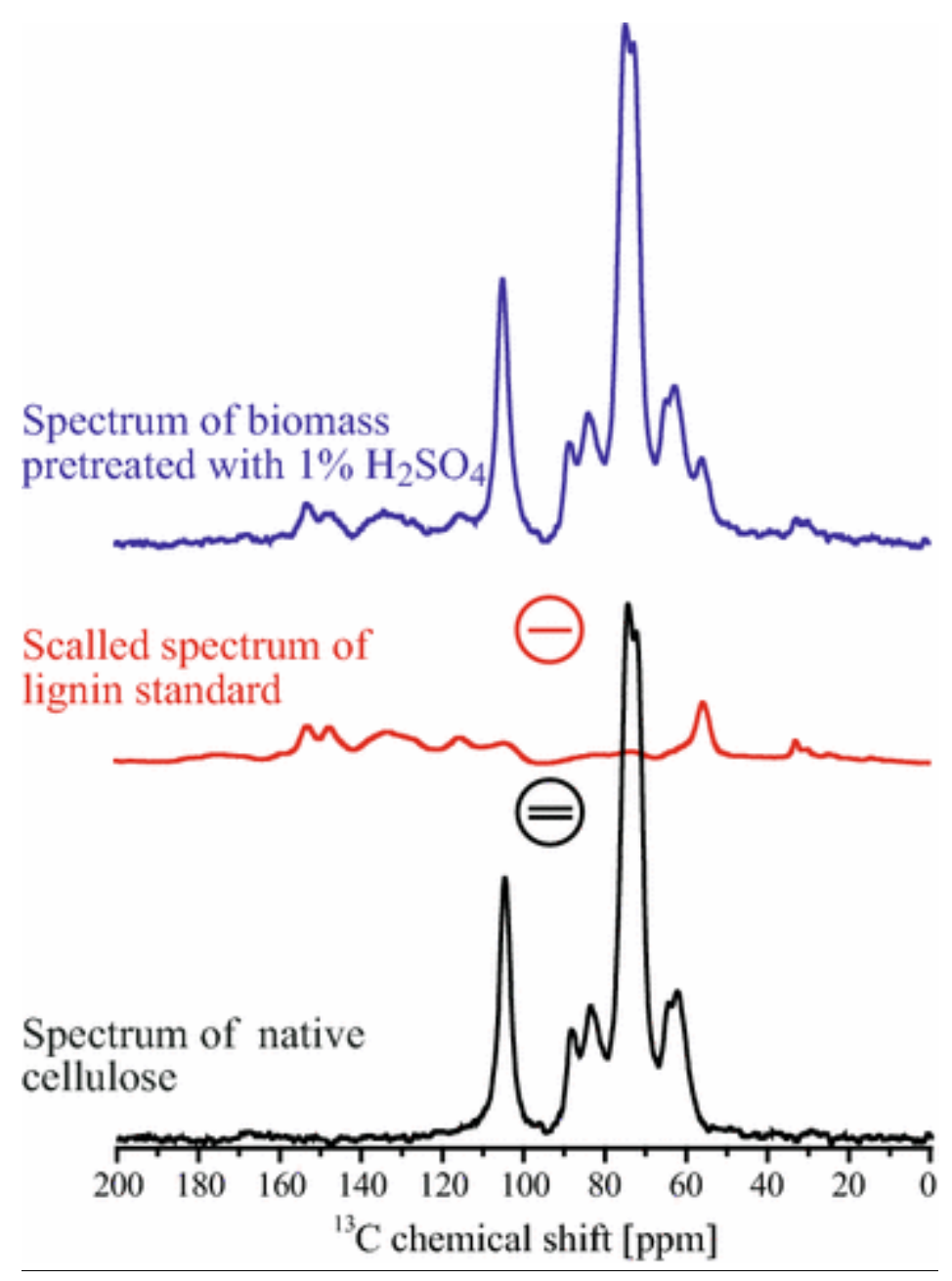

Figure 7. The spectrum of biomass pretreated with $1 \% \mathrm{H}_{2} \mathrm{SO}_{4}$ contains signals from both native cellulose and lignin (hemicellulose has been removed). Subtraction of a lignin spectrum provides a spectrum of native cellulose which can provide the crystalline index by focusing on the crystalline signal at $88 \mathrm{ppm}$ and on the amorphous signal at $84 \mathrm{ppm}$. Data was acquired at $9.4 \mathrm{~T}$ using a 14 $\mathrm{kHz}$ spinning frequency. Adapted with permission from Ref. [114]. Copyright 2015 Springer. 


\subsection{Heteronuclear correlation (HETCOR) spectroscopy}

It can prove very difficult to assign all the ${ }^{13} \mathrm{C}$ nuclei based only on the isotropic chemical shifts and signal intensities in $1 \mathrm{D}{ }^{13} \mathrm{C}\left\{{ }^{1} \mathrm{H}\right\}$ CP/MAS NMR experiments of samples with natural abundance ${ }^{13} \mathrm{C}$. A more precise assignment can be obtained by correlating protons $\left({ }^{1} \mathrm{H}\right)$ and carbons $\left({ }^{13} \mathrm{C}\right)$ in a $2 \mathrm{D}$ NMR experiment, which gives direct insight into the chemical structure. Subsequent to excitation of the ${ }^{1} \mathrm{H}$ spins, a typical heteronuclear correlation (HETCOR) experiment incorporates a homonuclear decoupling sequence in the indirect dimension that still allows the encoding of the chemical shift evolution of ${ }^{1} \mathrm{H}$ nuclei. After encoding ${ }^{1} \mathrm{H}$ isotropic chemical shifts, the magnetization is transferred typically by $\mathrm{CP}$ or LGCP to the ${ }^{13} \mathrm{C}$ nuclei whereupon the ${ }^{13} \mathrm{C}$ isotropic chemical shifts are encoded during acquisition under heteronuclear decoupling. We note that symmetry-based sequences $[115,116]$ can also be used to mediate magnetization transfer between ${ }^{1} \mathrm{H}$ and ${ }^{13} \mathrm{C}$, e.g. as in the PRESTO-HETCOR [117] experiment, which mainly correlate directly bonded protons and carbons [118].

If homonuclear decoupling is not invoked in the ${ }^{1} \mathrm{H}$ dimension, the resolution would be (very) low due to strong homonuclear dipolar couplings, at least for commonly employed MAS frequencies [119]. Some of the homonuclear decoupling sequences that yield acceptable resolution are FSLG [109, 110], PMLG [111], and the DUMBO [120, 121] family. In terms of nomenclature, it is common to denote the particular sequence in the name of the correlation experiment, e.g. FSLG-HETCOR. During the encoding of the indirect dimension, the ${ }^{1} \mathrm{H}$ carrier can be placed off resonance to benefit from "second 
averaging" effects of pulse imperfections, which further has the advantage that artifacts appearing at the carrier frequency will move outside the region of interest [122].

The before-mentioned homonuclear decoupling sequences have all been designed in the 'quasi-static' regime, where the rf field is supposed to complete the averaging on a timescale much shorter than the rotor period [123]. The consequence of applying a homonuclear decoupling ('quasi-static') sequence in the indirect dimension is that the chemical shifts are scaled to 0.58 of the original values. On the spectrometer, scaling factors below the theoretical limit of 0.58 can be the case requiring post-processing adjustment to the actual scaling. The scaling factor of 0.58 can easily be understood from the previous discussion on LG as the chemical shift tensor is projected onto the effective field axis at 54.7 degrees $(\cos (54.7)=0.58)$.

In Figure 3b, an example of the application of the 2D HETCOR NMR experiment was presented. The ${ }^{1} \mathrm{H}-{ }^{13} \mathrm{C}$ correlations eased the assignment of the ${ }^{1} \mathrm{H}_{\mathrm{br}}$ protons in $\mathrm{C}_{8,7}-\mathrm{PDI}$, thereby facilitating the determination of exact stacking distances between successive $C_{8,7^{-}}$ PDI molecules. More examples of $2 \mathrm{D}{ }^{13} \mathrm{C}\left\{{ }^{1} \mathrm{H}\right\}$ FSLG-HETCOR experiments can be viewed in Figure 8 where the donor-acceptor polymer poly[2,6-(4,4-bis-(2-ethylhexyl)-4Hcyclopenta[2,1-b;3,4-b']-dithiophene)-alt-4,7-(2,1,3-benzothiadiazole)] (PCPDTBT) in pristine films and in blends (1:2) with [6,6]-phenyl- $\mathrm{C}_{61}$-butyric acid methyl ester (PCBM) are examined [124]. The blends were processed with and without the solvent additive 1,8octanedithiol (ODT) and all materials were drop-cast from o-dichlorobenzene (o-DCB). These 2D HETCOR NMR experiments were performed to provide a morphological explanation of why the total internal quantum efficiency loss due to geminate 
recombination is ca. $50 \%$ in samples without ODT, while it is only $30 \%$ in samples with ODT.
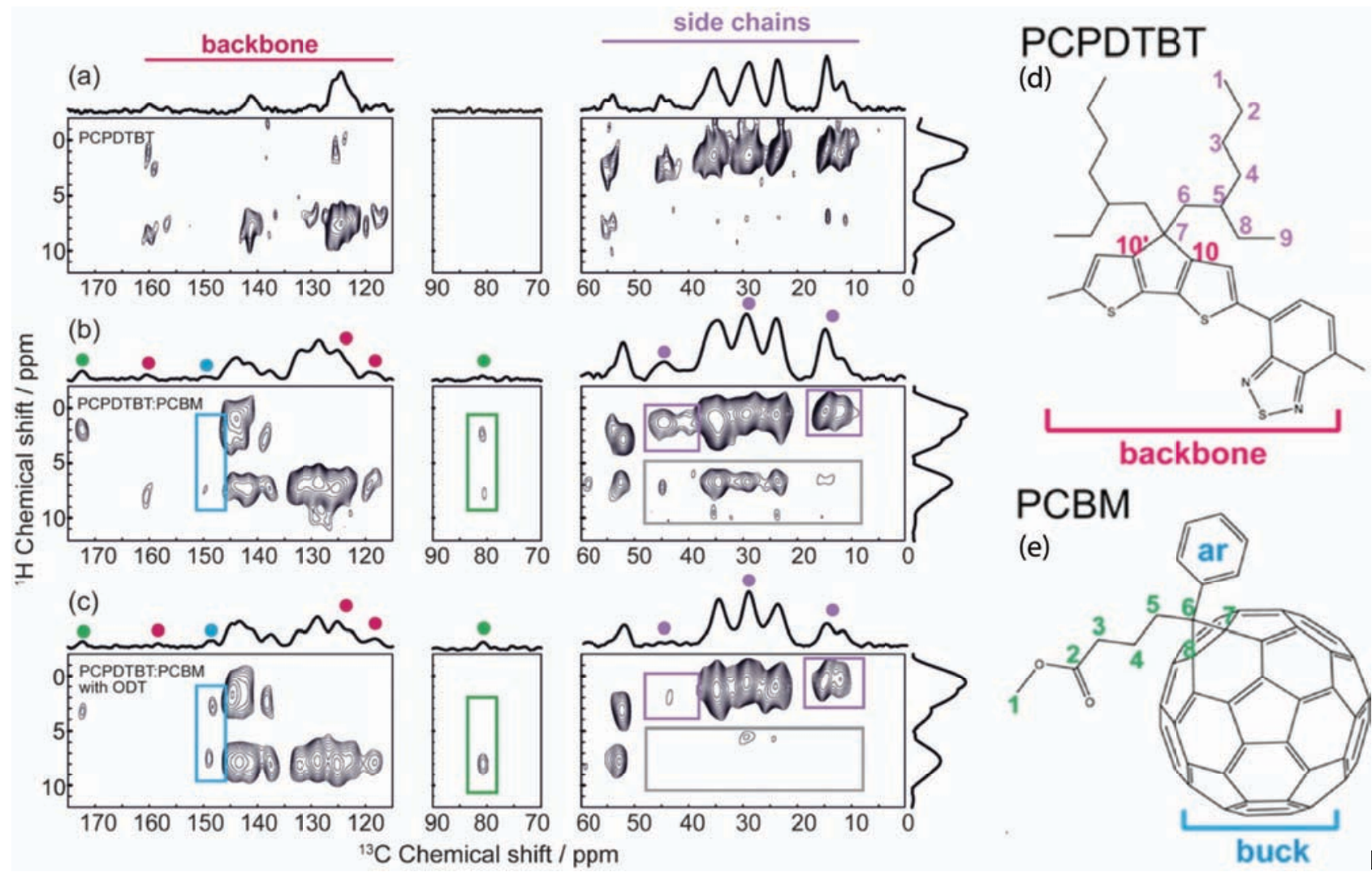

PCBM

(e)
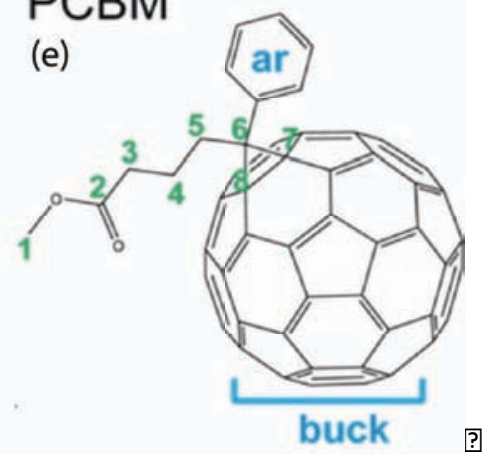

Figure 8. Selected regions of the $2 \mathrm{D}{ }^{13} \mathrm{C}\left\{{ }^{1} \mathrm{H}\right\}$ FSLG-HETCOR spectra of (a) pristine PCPDTBT, (b) PCPDTBT:PCBM (1:2) and (c) PCPDTBT:PCBM (1:2) processed with 2.4 vol. ODT. Assignment can be inferred from (d) and (e). The same intensity scale is used in all spectra with contour levels ranging from 10.1 to $90 \%$ of the maximum intensity. All data were acquired at $20.0 \mathrm{~T}$ and $15.0 \mathrm{kHz}$ MAS. Adapted with permission from Ref. [124]. Copyright 2013 American Chemical Society.

Comparing the 2D ${ }^{13} \mathrm{C}\left\{{ }^{1} \mathrm{H}\right\}$ FSLG-HETCOR spectra of pristine PCPDTBT (Figure 8a), PCPDTBT:PCBM (1:2) (Figure 8b) and PCPDTBT:PCBM (1:2) processed with 2.4 vol. ODT (Figure 8c), show that only correlations between aromatic protons and aliphatic carbons in PCPDTBT:PCBM without ODT (e.g. grey boxes) are observed. These correlations suggest that the PCPDTBT polymer and the PCBM moiety are molecularly mixed. In the PCPDTBT:PCBM blend processed with ODT, a signal intensity reduction in 
the aliphatic region compared to PCPDTBT:PCBM blend without ODT (purple boxes) is observed. Also, the signals are generally narrower in the former. Taken together these observations indicate that adding ODT to the blend leads to a higher degree of demixing with increased domain sizes as well as a higher degree of local order with better arranged intermolecular interfaces. Such material aspects are important for the further improvement of the power conversion efficiencies in organic solar cells [125].

\subsection{Medium- and long-distance heteronuclear correlation (MELODI- HETCOR) spectroscopy}

To divide the ${ }^{1} \mathrm{H}-{ }^{13} \mathrm{C}$ correlations into subsets of short- or long-range, several 2D HETCOR experiments are often performed with different (LG)CP mixing times. Those with short mixing times provide primarily short-range correlations (one-bond or close-in-space connections), whereas those with longer mixing times provide the structurally more interesting long-range range correlations, in addition to the short-range correlations. However, there is a great risk that the long-range range correlations are hidden or even obscured due to the more intense and dominating correlations from directly bonded ${ }^{1} \mathrm{H}-{ }^{13} \mathrm{C}$ spin pairs.

Yao et al presented the medium- and long-distance heteronuclear correlation (MELODIHETCOR) [122] experiment to circumvent this problem of the dominating short-range correlations. A schematic representation of the sequence can be seen in Figure 4c. The idea is to precede a 2D HETCOR experiment with a so-called MELODI-filter of two rotor

periods duration that dephases all ${ }^{1} \mathrm{H}$ spins that are directly bonded (or spatially very 
close) to a ${ }^{13} \mathrm{C}$ nucleus. The dephasing is achieved by recoupling of the heteronuclear dipole-dipole coupling interactions. The underlying mechanism relies on two $\pi$ pulses on the ${ }^{13} \mathrm{C}$ channel separated by a rotor period and one $\pi$ pulse in the middle of two rotor periods on the ${ }^{1} \mathrm{H}$ channel. The $\pi$ pulse on the ${ }^{1} \mathrm{H}$ spins serves to refocus the chemical shift interactions and to maintain the effect of the two $\pi$ pulses on the ${ }^{13} \mathrm{C}$ spins. In addition, FSLG decoupling suppresses the homonuclear ${ }^{1} \mathrm{H}-{ }^{1} \mathrm{H}$ dipolar interactions. The temporal placement of the two $\pi$ pulses on the ${ }^{13} \mathrm{C}$ spins is under the experimentalist's control. The objective is to dephase the magnetization of the ${ }^{1} \mathrm{H}$ nuclei directly bonded to a ${ }^{13} \mathrm{C}$ nucleus as much as possible, while trying to maintain the full magnetization of the other ${ }^{1} \mathrm{H}$ nuclei. In a way the MELODI-filter behaves much like a "variable-strength" REDOR experiment. However, the MELODI-HETCOR experiment is not suitable for fully ${ }^{13} \mathrm{C}$-labelled samples as basically all ${ }^{1} \mathrm{H}$ spins are attached to $a^{13} \mathrm{C}$ spin and will therefore be dephased during the filtering.

A simple Gedankenexperiment shows the utility of the MELODI approach on a naturally labeled system compared to a normal HETCOR setup. Suppose that ${ }^{13} \mathrm{C}_{\alpha}$ and ${ }^{12} \mathrm{C}_{\beta}$ is directly bonded to ${ }^{1} \mathrm{H}_{\alpha}$ and ${ }^{1} \mathrm{H}_{\beta}$, respectively. In such a system, 2D HECTOR shows a stronger ${ }^{1} \mathrm{H}_{\alpha}-{ }^{13} \mathrm{C}_{\alpha}$ correlation and a weaker ${ }^{1} \mathrm{H}_{\beta}-{ }^{13} \mathrm{C}_{\alpha}$ correlation. In a crowded $2 \mathrm{D}$ spectrum or in the case of overlap, the latter correlation would be hard to observe. This is the situation where MELODI-HETCOR comes into play. During the MELODI filter, which has been optimized to dephase the ${ }^{1} \mathrm{H}$ nuclei directly bonded to ${ }^{13} \mathrm{C}$ nucleus and to leave the rest untouched, the ${ }^{1} \mathrm{H}_{\alpha}$ magnetization will dephase while the ${ }^{1} \mathrm{H}_{\beta}$ magnetization is preserved. The isotropic chemical shift of ${ }^{1} \mathrm{H}_{\beta}$ is encoded in the indirect dimension whereupon the ${ }^{1} \mathrm{H}_{\beta}$ magnetization is transferred to ${ }^{13} \mathrm{C}_{\alpha}$, which is chemical shift encoded 
during acquisition. The resulting $2 \mathrm{D}{ }^{1} \mathrm{H}-{ }^{13} \mathrm{C}$ MELODI-HETCOR spectrum shows only the ${ }^{1} \mathrm{H}_{\beta}-{ }^{13} \mathrm{C}_{\alpha}$ correlation. Yao et al [122] noted that a LGCP transfer is advantageous over a normal CP if two- and three-bond correlations are the objective. If we on the other hand had put the MELODI filter at the end of the pulse sequence, the magnetization of ${ }^{1} \mathrm{H}_{\alpha}$ and of ${ }^{1} \mathrm{H}_{\beta}$ would have been chemical shift encoded and transferred to ${ }^{13} \mathrm{C}_{\alpha}$. However, the filter would subsequently destroy the ${ }^{13} \mathrm{C}_{\alpha}$ magnetization as the nucleus is directly attached to ${ }^{1} \mathrm{H}_{\alpha}$ and no correlation peak would appear in the spectrum.

\subsection{J-coupling based HETCOR spectroscopy}

In all of the above described 2D HETCOR experiments, the transfer of magnetization between a ${ }^{1} \mathrm{H}$ nucleus and a ${ }^{13} \mathrm{C}$ nucleus is driven by the heteronuclear dipole-dipole coupling interaction. To know for certain that two nuclei are directly bonded, one has to resort to J-coupling based experiments. The MAS-J-HMQC [126] (HMQC: heteronuclear multiple quantum coherences) experiment exploits the $\mathrm{J}$-coupling interaction under MAS conditions to obtain directly bonded proton-carbon correlations and is as such directly complimentary to the MELODI-HETCOR approach. A schematic representation of the sequence can be seen in Figure 4d and the underlying idea of MAS-J-HMQC can be appreciated by looking at the HMQC [127] experiment in a simplified version on spin $I\left({ }^{1} \mathrm{H}\right)$ and spin $S\left({ }^{13} \mathrm{C}\right)$. In the following overview, we have only included the most essential and illustrative features of the HMQC experiment. We start out with the magnetization on the $S$ spin which evolves under the $J$ coupling interaction for a $\tau$ period. The chemical shift will be refocused by a $\pi$ pulse. 


$$
S_{x} \stackrel{\pi J \tau 2 I_{z} S_{z}}{\longrightarrow} \oiint_{x} \cos (\pi J \tau) \quad 2 S_{y} I_{z} \sin (\pi J \tau)
$$

Here, we have used the well-known nomenclature in liquid-state NMR spectroscopy for evolution as dictated by $\stackrel{H t}{\longrightarrow}$. If $\tau$ is chosen properly, $\tau=1 /(2 J)$, we will only have the antiphase term. A $\pi / 2_{y}$ pulse, for instance, on the $I$ channel yields a DQ operator,

$$
2 S_{y} I_{z} \stackrel{\frac{\pi}{2} I_{y}}{\longrightarrow} 2 S_{y} I_{x}
$$

which encodes the chemical shift of $I$

$$
2 S_{y} I_{x} \stackrel{\omega_{1} I_{z}}{\longrightarrow} \mathbb{Z} S_{y} I_{x} \cos \left(\omega_{I} t_{1}\right) \quad 2 S_{y} I_{y} \sin \left(\omega_{I} t_{1}\right)
$$

Another $\pi / 2$ y pulse on the $I$ channel "restores" the antiphase term

$$
2 S_{y} I_{x} \cos \left(\omega_{I} t_{1}\right)+2 S_{y} I_{y} \sin \left(\omega_{I} t_{1}\right) \stackrel{\frac{\pi}{2} I_{y}}{\longrightarrow}-2 S_{y} I_{z} \cos \left(\omega_{I} t_{1}\right)+2 S_{y} I_{y} \sin \left(\omega_{I} t_{1}\right)
$$

which evolves back into magnetization (the latter term in Eq. (0.32) will not evolve under the $J$ coupling interaction)

$$
-2 S_{y} I_{z} \cos \left(\omega_{1} t_{1}\right) \stackrel{\pi J \tau 2 I_{z} S_{z}}{\longrightarrow} S_{x} \cos \left(\omega_{I} t_{1}\right)
$$

having used $\pi J \tau=\pi / 2$. The chemical shift of spin $S$ will be encoded during acquisition.

The MAS-J-HMQC does basically the same as the HMQC experiment but requires more demanding pulse sequence elements. The experiment starts with a CP element, thereby enhancing the initial magnetization on the ${ }^{13} \mathrm{C}$ spins (see Figure $4 d$ ). A $\pi$ pulse refocuses the chemical shift interaction up to the beginning of the acquisition. The FSLG decoupling elements decouples the strongly homonuclear coupled ${ }^{1} \mathrm{H}$ spins, and MAS averages chemical shift anisotropy and the heteronuclear dipolar coupling interactions. The 
magnetization evolves under the $J$ coupling interaction into antiphase. A $\pi / 2$ pulse renders it possible to encode ${ }^{1} \mathrm{H}$ isotropic chemical shifts under FSLG homonuclear decoupling. The DQ coherence is then turned back into observable in-phase magnetization by a $\pi / 2$ pulse and subsequent evolution under the $J$ coupling interaction. The cleverly placed "magic-angle pulses" increase sensitivity and minimize axial peaks. Note that the heteronuclear $\mathrm{J}$ couplings as well as the ${ }^{1} \mathrm{H}$ isotropic chemical shifts are both scaled by the factor of 0.58 due to the implementation of FSLG. Phase cycling has been invoked to select only the DQ heteronuclear coherences. As a technicality, Lesage et al [126] points

out that if the FSLG sequence yields line widths on the order of the heteronuclear $J$ coupling, then the experiment should be feasible. $\tau$ was chosen to be an integer number of the rotor period in Ref. [126] and should optimally be ca. $2 \mathrm{~ms}$.

\section{SYNTHETIC AND BIO-BASED POLYMERS}

The production of polymers without relying on oil resources is a major challenge to their sustainable application. This challenge has inspired researchers to take advantage of plant oils and other biomass-derived chemicals as feedstock for the production of bio-based polymers [128-131]. Another important strategy with the potential of meeting this challenge is to take advantage of enzymatic reactions, facilitating a green chemistry route [132]. However, whether the polymers synthesized using either of these methods have comparable chemical and physical properties to those made from petrochemical sources is a concern. From a structural point-of-view, the points of interest are (i) how the polymer material crystallizes to form nano-structured domains [133], utilizing non-covalent 
interactions such as hydrogen-bonding and $\pi-\pi-s t a c k i n g ~[134-136]$, (ii) crystallinity and chain conformations [137], and (iii) whether the bio-based groups are conformationally stable when incorporated into a polymeric structure. These important structural properties can influence the melting point and lead to potentially stiffer or more flexible materials.

\subsection{Influence of ester groups on the physical properties of polyolefins}

In a recent study focusing on the incorporation of ester groups in polyethylene, the crystalline and amorphous domains of a polymer have been characterized with the aim of quantifying the partitioning of the ester groups [138]. The aliphatic long-chain polyesters (ALCPEs) were synthesized via ring-opening metathesis polymerization (ROMP) of cyclic olefins [139-141]. Figure 9 displays the melting temperatures of a series of ALCPEs plotted as a function of the methylene-to-ester ratio $(\mathrm{M} / \mathrm{E})$, illustrating that an increase of the number of methylene units between the ester groups causes a gradual increase in the melting temperature. At high M/E ratios, the melting temperature coincides with that of high density polyethylene (HDPE). We note that a similar temperature behavior has been observed in a polyethylene sample with aliphatic side chains incorporated at well-defined positions [142, 143]. 


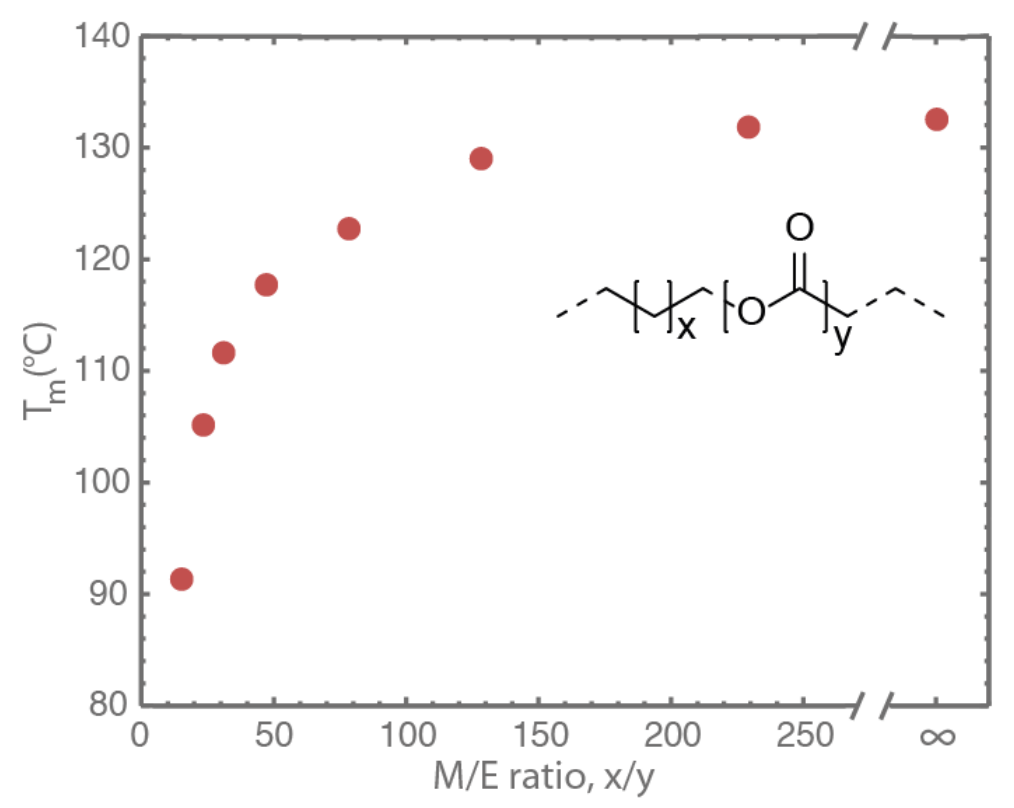

Figure 9. Plot of the melting temperature for ALCPEs as a function of the methylene-to-ester ratio (M/E) synthesized via ROMP using a first-generation Grubbs catalyst. Adapted with permission from Ref. [138]. Copyright 2013 American Chemical Society.

To characterize the partitioning of the ester groups over the crystalline and amorphous phases in a ALCPE sample with a M/E ratio of 31 , a combination of NMR experiments were employed. This included variable-temperature (VT) solid-state ${ }^{13} \mathrm{C}\left\{{ }^{1} \mathrm{H}\right\}$ INEPT MAS NMR, ${ }^{13} \mathrm{C}\left\{{ }^{1} \mathrm{H}\right\}$ CP/MAS NMR, and quantitative ${ }^{13} \mathrm{C}$ MAS NMR together with liquid-state ${ }^{13} \mathrm{C}$ NMR (see Figure S8 in Ref. [138]). The spectra from these experiments are summarized in Figure 10, which can be used to differentiate between the rigid and flexible portions of the ALPCE sample. This is because these portions experience motions with different correlation times, as described in Section 3.6 (Figure 6). The two main ${ }^{13} \mathrm{C}$ resonances at 30.8 and $32.8 \mathrm{ppm}$ are assigned to the non-crystalline and the crystalline all-trans methylene conformations, respectively [144]. The a-methoxy carbons also display two signals: one at $64.3 \mathrm{ppm}$ arising from the more flexible fraction in gauche conformation, as can be identified via the ${ }^{13} \mathrm{C}\left\{{ }^{1} \mathrm{H}\right\}$ INEPT MAS NMR spectra in Figure 10a, and a second 
peak at $65.7 \mathrm{ppm}$ from the crystalline part that gradually decreases upon heating (cf. Figure $10 \mathrm{a}$ and $\mathrm{b}$ ). The intensity of the carbonyl signal at $172.9 \mathrm{ppm}$ also decreases upon heating of the ALPCE sample. However, since a carbonyl has no directly bonded protons, no additional information about the flexible carbonyl fraction can be obtained via ${ }^{13} \mathrm{C}\left\{{ }^{1} \mathrm{H}\right\}$ INEPT NMR (Figure 10a). For this reason, we have used the amorphous and crystalline ${ }^{13} \mathrm{C}$ signals of the a-methoxy carbon and the methylene groups to quantify the partitioning of ester groups between the crystalline and amorphous phases as illustrated in Figure 10c. This spectrum was recorded using a single-pulse ${ }^{13} \mathrm{C}$ MAS NMR experiment under quantitative conditions, employing a small excitation flip angle of $30^{\circ}$, a relaxation delay of $30 \mathrm{~s}$, and high-power ${ }^{1} \mathrm{H}$ decoupling during acquisition. A deconvolution of the ${ }^{13} \mathrm{C}$ signals in both mentioned regions in Figure 10c yields ratios of 1:1.14 and 1:1.18 for the amethoxy and methylene groups, respectively. Thus, a strategic combination of solid-state and liquid-state NMR methods demonstrates that the ester groups are uniformly distributed over the crystalline and amorphous phases, which is in agreement with results from DSC and X-ray diffraction experiments [138]. 


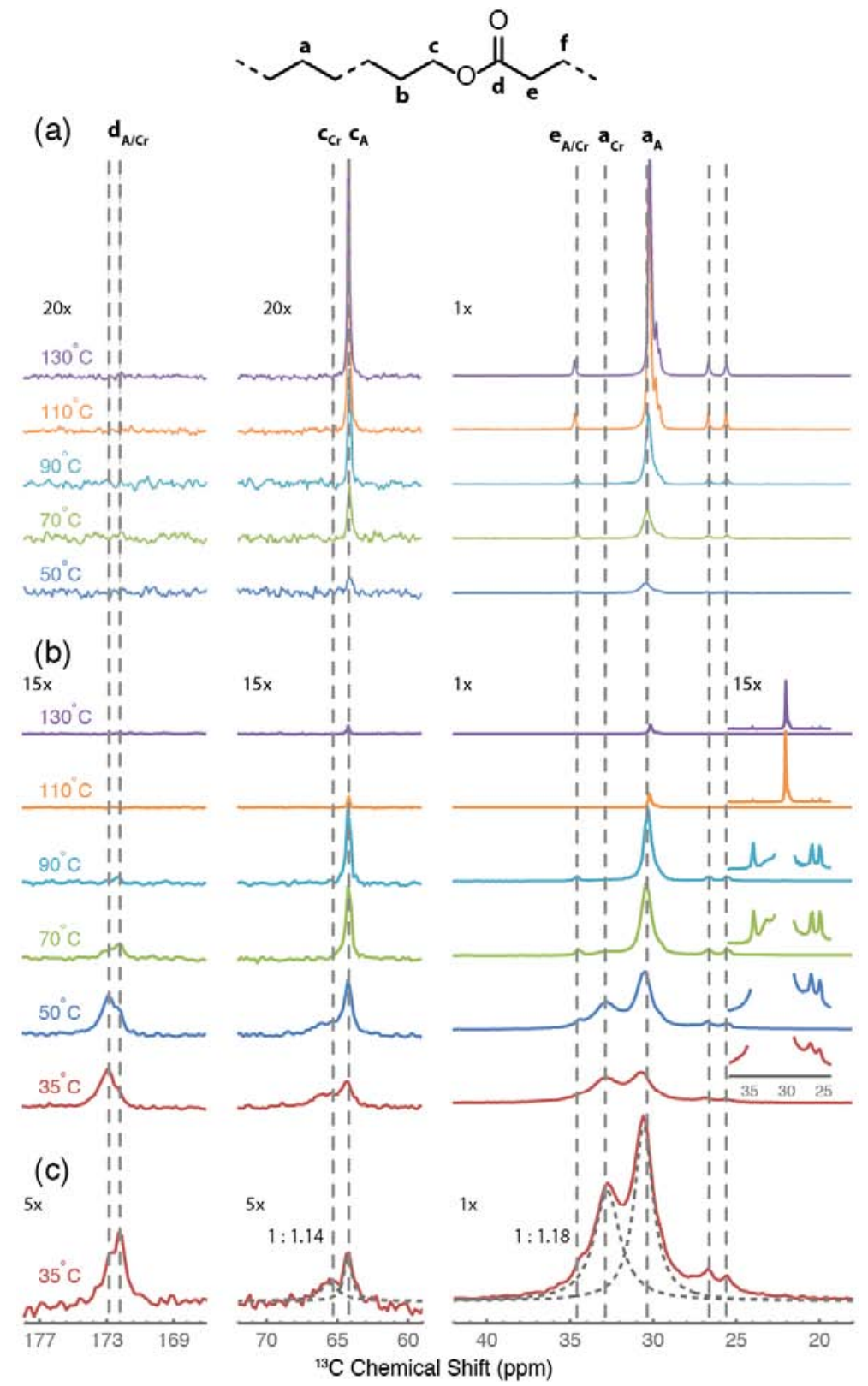

Figure 10. Variable-temperature (a) ${ }^{13} \mathrm{C}\left\{{ }^{1} \mathrm{H}\right\}$ INEPT MAS NMR, (b) ${ }^{13} \mathrm{C}\left\{{ }^{1} \mathrm{H}\right\} \mathrm{CP} / \mathrm{MAS}$ NMR and (c) ${ }^{13} \mathrm{C}$ MAS NMR spectra of an aliphatic long-chain polyesters (ALCPE) with a methylene-to-ester ratio of 31 (see inset of Figure 9) recorded from 35 to $130{ }^{\circ} \mathrm{C}$. The quantitative ${ }^{13} \mathrm{C}$ MAS NMR spectrum in (c) includes a deconvolution into crystalline $(\mathrm{Cr})$ and amorphous $(\mathrm{A})$ fractions (assigned using dashed lines) for the methylene and a-methoxy groups. Adapted with permission from Ref. [138]. Copyright 2013 American Chemical Society. 


\subsection{Bio-based polyamides}

Polyamides are the most important class of step-growth polymers due to their favorable and controllable physicochemical and mechanical properties [145]. For this reason they are extensively used in industry for injection molding, extrusion, and film or fiber applications. Recently, we have characterized a series of bio-based polyamides synthesized by incorporation of the carbohydrate-derived 1,4:3,6-dianhydrohexitols, also known as isohexides, since these are suitable building blocks for step-growth polymerization [146-148]. Isohexides are secondary diols derived from C6-sugars that can be obtained from starch or cellulose via a few (bio-)organic transformations [149]. Three different isohexide isomers exist, where the major conformer for each isomer is shown in Figure 11a. These isohexide isomers give rise to quite different ${ }^{13} \mathrm{C}$ chemical shifts as illustrated in Figure 11b obtained from gas-phase quantum-chemical calculations (MP2/6$\left.311 G^{\star *}\right)[148]$. 
(a)

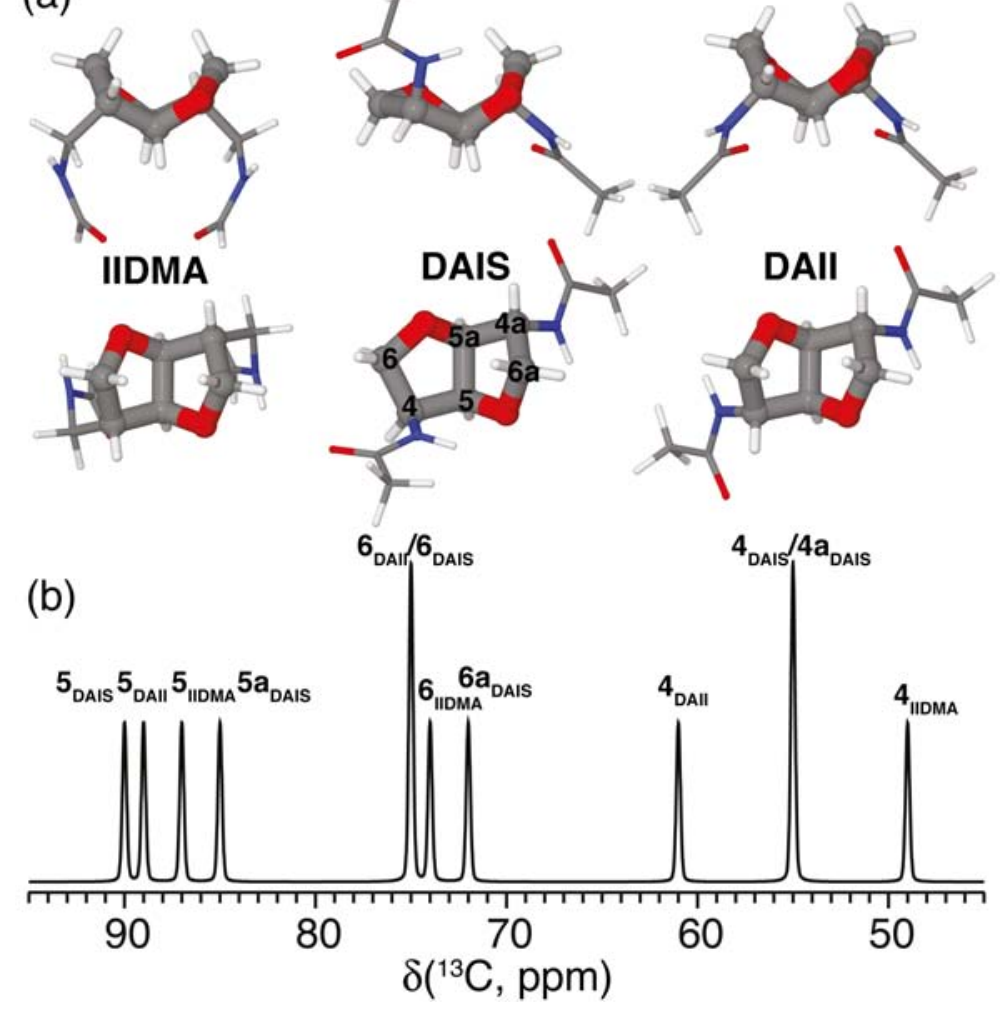

Figure 11. (a) Top and side views of the most stable conformers for the primary diamines isoidide2,5-dimethyleneamine (IIDMA, endo-endo), diaminoisosorbide (DAIS, exo-endo), and diaminoisoidide (DAll, exo-exo) determined via gas-phase MP2/6-311G** calculations. (b) Graphical representation of the calculated ${ }^{13} \mathrm{C}$ chemical shifts for all three conformers assigned using the nomenclature shown for the DAIS conformer in part (a). Adapted with permission from Ref. [148]. Copyright 2012 American Chemical Society.

To study the solid-state behavior and stability of the isohexide isomers incorporated into polyamides, VT solid-state ${ }^{13} \mathrm{C}\left\{{ }^{1} \mathrm{H}\right\}$ CP/MAS NMR experiments have been employed [146148]. As reported by Gitsas et al. [150], the dynamic changes of the local chain mobility of a polymer result in the reduction of the effective nuclear dipole-dipole couplings and thereby a lower $\mathrm{CP}$ efficiency at higher temperatures. Thus, by affecting the hydrogen bonding of the nano-structured polyamide chain fragments, the intensity of the ${ }^{13} \mathrm{C}$ signals originating from the crystalline phase will be affected more rapidly as a function of 
temperature compared to ${ }^{13} \mathrm{C}$ signals from the amorphous domains. As an example of these isohexide-based polyamides, Figure 12a displays a series of VT solid-state ${ }^{13} \mathrm{C}\left\{{ }^{1} \mathrm{H}\right\}$ CP/MAS NMR spectra for diaminoisosorbide (DAIS) when incorporated into a regular polyamide structure based on sebacic acid monomers. The ${ }^{13} \mathrm{C}\left\{{ }^{1} \mathrm{H}\right\} \mathrm{CP} / \mathrm{MAS}$ NMR spectra exhibit well-resolved ${ }^{13} \mathrm{C}$ signals from the $\mathrm{C} 4$ and $\mathrm{C} 4 a$ positions directly attached to the exo- and endo-oriented amide groups of DAIS (see Figure 12a). This suggests that the polymerization reaction does not affect the stereo configuration of the DAIS group. Moreover, the ${ }^{13} \mathrm{C}$ resonances $\mathrm{C} 5, \mathrm{C} 5 \mathrm{a}$, and the amide $\mathrm{C}=\mathrm{O}$ at $174.6 \mathrm{ppm}$ exhibit lower intensities and become shifted to higher frequencies upon heating. The signals from $\mathrm{C} 5$, C5a, C6, and C6a display significant changes in their intensities close to the melting point. The coexistence of these signals close to the melting point reflects the distribution of DAIS residues over the crystalline and amorphous domains of the sample. More significant intensity changes of the $\mathrm{C} 5, \mathrm{C} 6$, and $\mathrm{C} 4$ signals are observed above $100^{\circ} \mathrm{C}$, emphasizing the distribution of DAIS residues in both phases of the semi-crystalline polymer sample. 
(a)

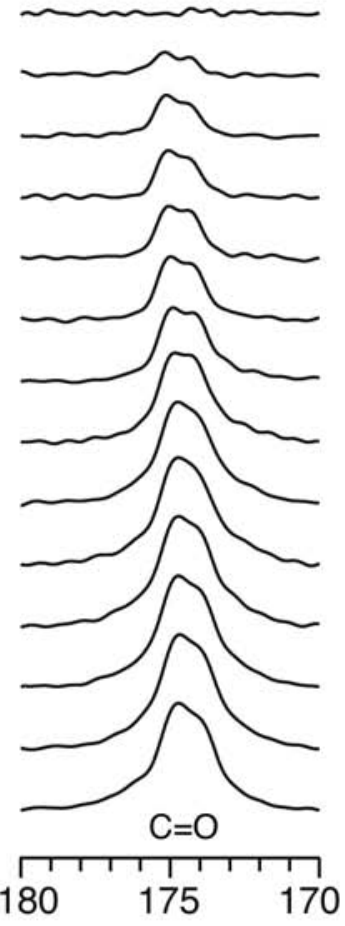

(b)

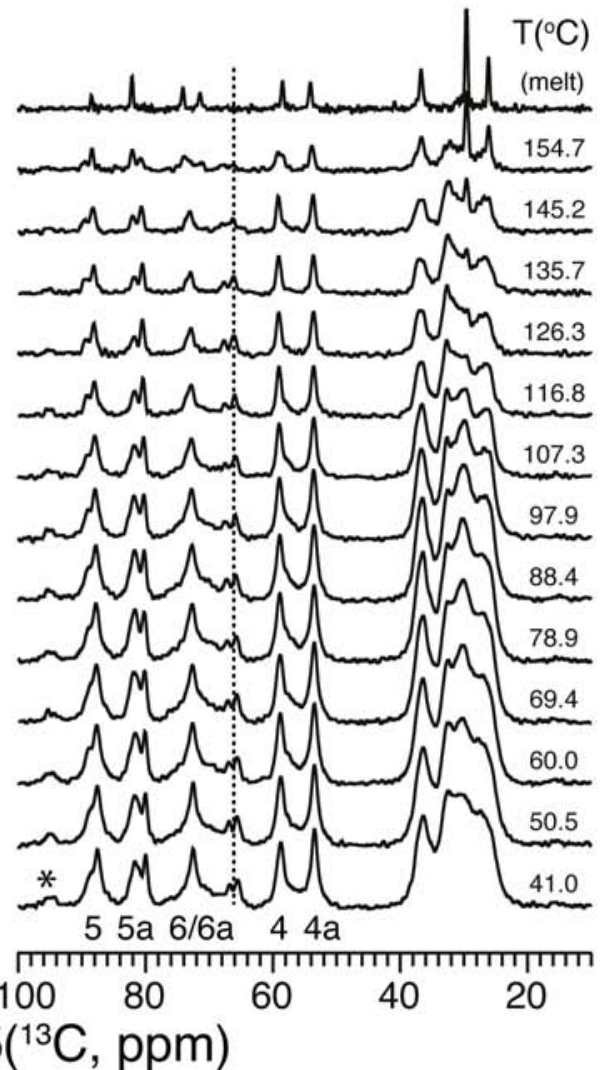

(c)
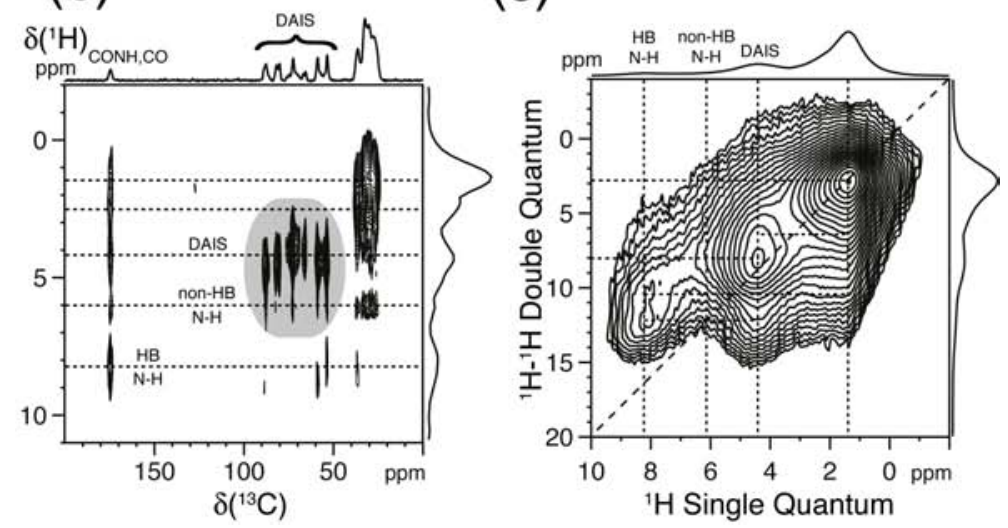

Figure 12. (a) Variable-temperature solid-state ${ }^{13} \mathrm{C}\left\{{ }^{1} \mathrm{H}\right\}$ CP/MAS NMR spectra of a bio-based polyamide synthesized from sebacic acid and diaminoisosorbide (DAIS). (b) $2 \mathrm{D}{ }^{13} \mathrm{C}\left\{{ }^{1} \mathrm{H}\right\}$ FSLGHETCOR spectrum acquired using a CP time of $2.0 \mathrm{~ms}$. (c) $2 \mathrm{D}{ }^{1} \mathrm{H}-{ }^{1} \mathrm{H} D Q-S Q$ spectrum recorded using a Back-to-Back (BaBa) recoupling/reconversion period of $67.2 \mu \mathrm{s}$. The dashed lines in (b) and (c) illustrate selected cross-peaks and autocorrelation peaks, including hydrogen-bonded (HB) and non-hydrogen-bonded (non-HB) amide fragments. Assignment is performed according to the scheme given in Figure 11a. The asterisk indicates the position of a spinning sideband from the carbonyl resonance. Adapted with permission from Ref. [147]. Copyright 2012 American Chemical Society. 
Details about the molecular packing and hydrogen-bonding structure of the DAIS-based polyamide can be derived from $2 \mathrm{D}{ }^{13} \mathrm{C}\left\{{ }^{1} \mathrm{H}\right\}$ FSLG-HETCOR and ${ }^{1} \mathrm{H}-{ }^{1} \mathrm{H}$ DQ-SQ correlation experiments as shown in Figure 12b and c. These 2D NMR spectra show that the DAISbased polyamide includes two different types of amide groups, which are related to hydrogen-bonded (8.3 ppm) and non-hydrogen-bonded groups (6.0 ppm), as seen in Figure 12b. The existence of these two hydrogen-bonding environments can also be identified from Figure $12 \mathrm{a}$, since a doubling of the carbonyl resonance is observed. Most likely the two different hydrogen-bonding environments are related to the non-crystalline and crystalline regions of the sample; however, no clear evidence for this behavior can be derived from the VT ${ }^{13} \mathrm{C}\left\{{ }^{1} \mathrm{H}\right\}$ CP/MAS NMR spectra in Figure 12a, where both carbonyl signals show the same intensity decay as a function of temperature. However, the 2D ${ }^{1} \mathrm{H}-{ }^{1} \mathrm{H}$ DQ-SQ correlation spectrum in Figure $12 \mathrm{~b}$ shows that the hydrogen-bonding environments are spatially separated (no cross-peak detected) and that the missing autocorrelation between the hydrogen-bonded amide groups points to a chain-folded structure, where these groups are not in close proximity within the sheet-like packing of the folded polyamides chains. Thus, on the basis of temperature-dependent solid-state NMR experiments discussed above, combined with WAXD and DSC analysis, it is possible to show that the crystallinity, and thereby the physical properties of the investigated compositions, can be tailored by the content of the bicyclic diamine in the backbone of the polyamides [146-148]. 


\section{SILKS}

Silk is a fibrous protein that is produced in nature by a variety of arthropods. Natural silks are of variable mechanical strengths, largely depending on their use by the source, and are produced under relatively benign conditions with low energy costs [151]. Along with its biocompatibility, silk has routinely been highlighted as a material with high strength and toughness [152], properties that are imparted during their processing by the source [153]. To uncover the underlying reasons for these emergent properties, the structural features of a variety of silks have been the subject of much research effort. Silks are semi-crystalline at best, and are constituted of a mixture of protein secondary structures and polymorphs. Much of the earlier work on structural characterization of silks relied on diffraction methods [154], exclusively focusing on the crystalline domains of the fibroins due to inherent limitations of the techniques. In contrast, solid-state NMR provides a potential method to acquire an atomic level view of silk fibroins in their native states, without a need for special sample treatment. Hence, by utilizing established methods of isotopically enriching natural proteins and peptides, an accurate picture of the silk structure can be produced. NMR has been used to study silks from bees [155], mantises [156], hornets [157], caddisflies [158] and webspinners [159]. In this contribution, however, some of the structure elucidation efforts of silkworm and spider native silk fibroins will be focused on exclusively to highlight the utility of solid-state NMR techniques. Furthermore, since solid-state NMR is a powerful technique to study dynamics and conformational changes in materials [38], an application of this to wet spider silks undergoing "supercontraction" will also be discussed. 


\subsection{Protein labeling strategies}

The solid-state ${ }^{1} \mathrm{H}$ MAS NMR spectra of proteins usually exhibit large spectral overlap of broad signals, owing to proton's short chemical shift range and large ${ }^{1} \mathrm{H}-{ }^{1} \mathrm{H}$ homonuclear dipolar couplings. Protons lack the resolving power of ${ }^{13} \mathrm{C}$ and ${ }^{15} \mathrm{~N}$, which typically have very large chemical shift ranges. However, due to low natural abundance and weak sensitivity of these nuclei, limited information can be gleaned from isotopically unenriched samples. Hence, isotopic enrichment of proteins is essential to be able to run advanced solid-state NMR experiments to produce a highly accurate picture of their structure.

To enrich silk fibroins, different methods are used depending on the silk source. For silkworms, feeding of an artificial diet that is enriched in an isotopically enriched amino acid is typically employed [160]. The resulting enriched silks are then gathered and prepped for analysis. For spiders, since dragline silk is typically the focus of study, forced reeling is employed during which an aqueous solution of enriched amino acids [161] or sugars [162] are fed while the silk is collected.

The target amino acids are usually chosen based on an apriori knowledge of some of the relevant structural details [163]. These methods require a carefully selected labeling scheme to counteract the effects of isotope scrambling and amino acid conversion, which might randomize the isotopic label within the protein $[163,164]$.

\subsection{Silkworm silk fibroin}

Silkworm silk fibroin is composed of a crystalline motif that is made up of multiple (Alanine (Ala)-Glycine (Gly)-Serine (Ser)-Gly-Ala-Gly) ${ }_{n}$ repeats, and a Tyrosine (Tyr) rich region [165]. ${ }^{13} \mathrm{C}\left\{{ }^{1} \mathrm{H}\right\}$ CP/MAS NMR has been utilized extensively to study these fibroins as ${ }^{13} \mathrm{C}$ 
chemical shifts are sensitive to the local environment, and can thus, report on the secondary structure. One of the first solid-state NMR studies of silkworm fibroin dimorphs, silk I and silk II, was undertaken by Saito et al. [160] using ${ }^{13} \mathrm{C}\left\{{ }^{1} \mathrm{H}\right\} \mathrm{CP} / \mathrm{MAS}$ NMR experiments. Silk I is the dried form of the fibroin produced by the middle silk gland of silkworms, while silk II is obtained after spinning. Silk I is essentially a precursor to silk II, with the structural transition from silk I to silk II only affecting the crystalline region of the silkworm silk fibroin [165]. Hence, attempts to impart crystallinity via macroscopic orientation often led to the conversion of silk I to silk II [154]. ${ }^{13} \mathrm{C}\left\{{ }^{1} \mathrm{H}\right\} \mathrm{CP} / \mathrm{MAS}$ NMR allowed for the interrogation of silk I without any need for extensive material transformation. They reported that the chemical shifts of the silk I amino acid residues of Ala and Gly did not match with those of model polypeptides known to be in an a-helix structure, nor those in a $\beta$-sheet. Instead, they concluded that a loose helix best approximates the most probable structure. Asakura et al. [166] refined these findings using a combination of $2 \mathrm{D}{ }^{13} \mathrm{C}$ spin diffusion and ${ }^{13} \mathrm{C}\left\{{ }^{15} \mathrm{~N}\right\}$ REDOR NMR on a model polypeptide, Poly(Ala-Gly). Note that this polypeptide lacks Ser that is present in the silk fibroin. However, Ser was known not to affect the chemical shifts of Aly and Gly residues in the fibroin $[166,167]$. The 2D ${ }^{13} \mathrm{C}$ spin diffusion NMR spectra of particular ${ }^{13} \mathrm{C}$ labeled Ala and Gly polypeptides showed good match with simulations at $\Phi=-60^{\circ}$ and $\Psi=130^{\circ}$ for the Ala residue, and $\Phi=70^{\circ}$ and $\Psi=10^{\circ}$ for the Gly residue. In combination with ${ }^{13} \mathrm{C}-{ }^{15} \mathrm{~N}$ REDOR, as well as independently performed ${ }^{13} \mathrm{C}-{ }^{2} \mathrm{H}$ REDOR experiments [168], a repeated type II $\beta$-turn structure was proposed for the crystalline part of the silk I backbone structure. 
In terms of the silk II secondary structure, since it is easier to handle than silk I, fiber diffraction studies had long proposed it to be mainly of an antiparallel $\beta$-sheet character [169]. Saito et al. [160], using an approach similar to what they used with silk I, agreed with the $\beta$-sheet conformer model. However, it was also recognized that some disorder existed in the crystalline Ala-Gly region [170]. To characterize the fibroin more precisely, Asakura et al. [165] performed ${ }^{13} \mathrm{C}\left\{{ }^{1} \mathrm{H}\right\} \mathrm{CP} / \mathrm{NMR}$ experiments on isotopically enriched native fiber and model polypeptides. The broad and asymmetric peak of the Ala $C_{\beta}$ around $20 \mathrm{ppm}$, the black line in Figure 13, points to the heterogeneous nature of the structure. Deconvolution of peaks from the crystalline region suggested the presence of a distorted $\beta$-turn (18\%), and $\beta$-sheet A (alternating Ala residues) (25\%) and B (parallel Ala residues) (13\%) structures. The amorphous Tyr rich region was found to contain equal numbers of a distorted $\beta$-turn and distorted $\beta$-sheet. As noted before, these details have not been accessible from previous X-ray analyses.

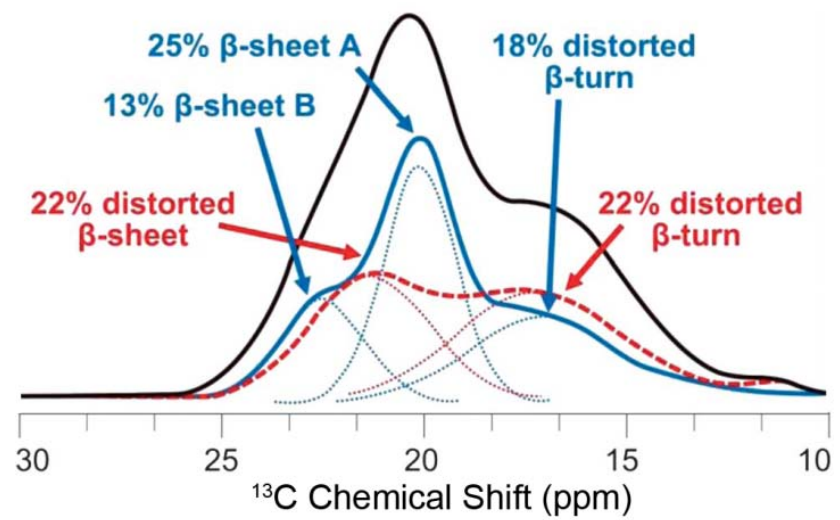

Figure 13. ${ }^{13} \mathrm{C}$ Ala $\mathrm{C}_{\beta}$ peak of the ${ }^{13} \mathrm{C}$ CP/MAS spectrum of ${ }^{13} \mathrm{C}$ enriched silkworm fibroin fiber. Deconvolution of the peak into conformations are shown. Adapted with permission from Ref. [171]. Copyright 2013 The Royal Society of Chemistry. 


\subsection{Spider major ampullate dragline silk fibroin}

Spiders need to produce a variety of silks for different functions [172]. Major ampullate dragline silk possesses an enviable combination of strength and elasticity [172, 173], and has particularly been the subject of sustained research. It is known that dragline silk contains a large amount of Gly and Ala residues, as well as some levels of glutamine (Glu), leucine (Leu), Ser, Tyr, asparagine (Asn) and proline (Pro) [174, 175]. Structurally, the dragline silk fibroin is composed of two protein fibers, namely MaSp1 and MaSp2 [176, 177]. The different ratios of these fibers, in part, affect the mechanical properties of dragline silk from different species [174].

MaSp1 contains numerous Leu-Gly-X-Glu (X = Ser, Gly, or Asn) throughout the fibroin, which flank the crystalline poly(Ala) and poly(Gly-Ala) sequences. The Jelinsky group provided valuable structural insight of the fibroin using solid-state ${ }^{2} \mathrm{H}[175]$ and ${ }^{13} \mathrm{C}-{ }^{15} \mathrm{~N}$ REDOR NMR [164]. ${ }^{2} \mathrm{H}$ solid-state NMR of the oriented silk fibers reported contributions from highly and weakly oriented fractions. It was calculated that $37 \%$ of Ala occupied the highly oriented fraction, while $63 \%$ occupied the weakly oriented fraction. The weakly oriented was speculated to effectively couple the crystalline and amorphous regions, explaining the toughness of the material. ${ }^{13} \mathrm{C}-{ }^{15} \mathrm{~N}$ REDOR NMR [164], on the other hand, showed that the Leu-Gly-X-Gln ( $\mathrm{X}=\mathrm{Ser}$, Gly, or Asn) motif in the dragline silk form turnlike heterogeneous structures, allowing the fibroin to assume a very compact architecture. Other structures, such as a disordered $3_{1}$-helical structure at the Gly-Gly-X motifs, have also been shown to exist by solid-state NMR methods [178].

The MaSp2 protein is characterized by Gly-Pro-Gly-X-X regions flanking poly(Ala) $\beta$ sheets. Interestingly, the difference in chemical shifts of Pro $C_{\beta}$ and $C_{Y}$ can report on the 
secondary structure, due to its sensitivity to local conformation [179-181]. Jenkins et al. [174] used ${ }^{13} \mathrm{C}-{ }^{13} \mathrm{C}$ Dipolar Assisted Rotational Resonance (DARR) NMR to characterize the secondary structure of the Pro rich region. Figure 14 shows the ${ }^{13} \mathrm{C}\left\{{ }^{1} \mathrm{H}\right\}$ CP/MAS NMR spectrum of the dragline silk of the ${ }^{13} \mathrm{C} /{ }^{15} \mathrm{~N}$-proline enriched Argiope aurantia spider, which is rich in MaSp2. However, extensive spectral overlap between Pro and other amino acids, with the exception of the Pro $C_{a}$, compromises the extraction of exact chemical shift values. Hence, ${ }^{13} \mathrm{C}-{ }^{13} \mathrm{C}$ correlation NMR with a medium 50 ms DARR recoupling period was utilized to exploit the connectivity within the Pro residue to yield precise chemical shifts. A chemical shift difference of $5.1 \mathrm{ppm}$ was found, which was indicative of a type II $\beta$-turn structure. This structural assignment was further confirmed by using ${ }^{15} \mathrm{~N}-{ }^{13} \mathrm{C}$ HETCOR experiments, since the ${ }^{15} \mathrm{~N}$ chemical shift of Pro is sensitive to backbone conformations.

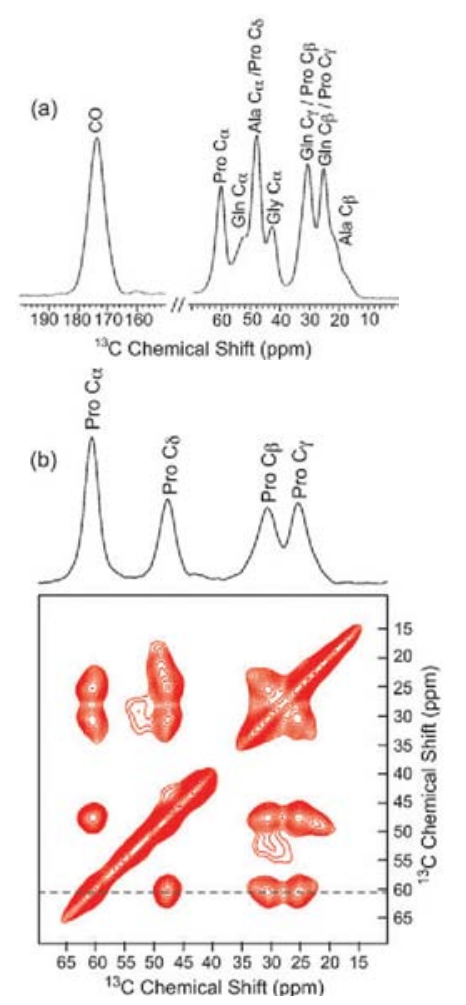

Figure 14. (a) ${ }^{13} \mathrm{C}\{\mathrm{H}\} \mathrm{CP} / \mathrm{MAS}$ NMR spectrum and (b) $2 \mathrm{D}^{13} \mathrm{C}-{ }^{13} \mathrm{C}$ NMR correlation spectrum with a $50 \mathrm{~ms}$ DARR recoupling period of ${ }^{13} \mathrm{C} /{ }^{15} \mathrm{~N}$ enriched spider dragline silk. The dotted line in (b) marks the correlations experienced by the Pro $C_{a}$. Adapted with permission from Ref. [182]. Copyright 2010 The Royal Society of Chemistry. 


\subsection{Dynamics in spider dragline silk probed using solid-state NMR}

MaSp2 is primarily implicated in the "supercontraction" process of spider dragline silk, wherein the silk decreases in length by $60 \%$ upon wetting [172], leading to a drastic change in mechanical properties [183]. Pro residues (ubiquitous in MaSp2, but not in MaSp1) are thought to play a central role in interacting with water. This interaction leads to a plasticizing effect in the surrounding structures, which possibly changes the crystal cross-link density and hydrogen bonding network [183]. Jenkins et al. [174] examined the effect of water on a spider silk rich in MaSp2 using ${ }^{13} \mathrm{C}\left\{{ }^{1} \mathrm{H}\right\}$ CP/MAS and direct detection (DD) MAS ${ }^{13} \mathrm{C}$ NMR with a short 1 sec delay. While ${ }^{13} \mathrm{C}\left\{{ }^{1} \mathrm{H}\right\} \mathrm{CP} / \mathrm{MAS}$ would enhance signals from rigid residues, the mobile regions will be enhanced in the rapid ${ }^{13} \mathrm{C}$ DD/MAS NMR. Figure 15 shows the ${ }^{13} \mathrm{C}\left\{{ }^{1} \mathrm{H}\right\} \mathrm{CP} / \mathrm{MAS}$ and ${ }^{13} \mathrm{C}$ DD/MAS NMR spectra of dry and wetted spider dragline silk. The Pro residues (as well as its flanking Gly residues in the MaSp2 motif) experience a significant reduction in intensity when interrogated with ${ }^{13} \mathrm{C}\left\{{ }^{1} \mathrm{H}\right\}$ CP/MAS NMR (Figure 15a), signifying an increase in mobility. Concomitantly, these residues experience a strong enhancement in the ${ }^{13} \mathrm{C}$ DD/MAS spectrum (Figure 15b), confirming the Pro residue's interaction with water in the wet, supercontracted state. Creager et al. [182] performed similar ${ }^{13} \mathrm{C} \mathrm{CP}$ and DD/MAS experiments on five different spider species, arriving at the same conclusion that silks high in Pro content plasticized more compared to those that did not. 


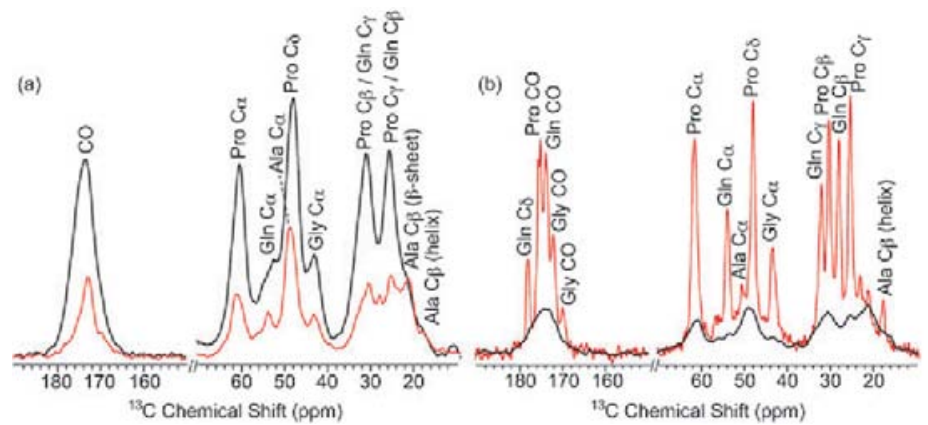

Figure 15. (a) ${ }^{13} \mathrm{C}\left\{{ }^{1} \mathrm{H}\right\} \mathrm{CP} / \mathrm{MAS}$ NMR spectra and (b) ${ }^{13} \mathrm{C}$ DD/MAS spectra of dry (black) and wet, supercontracted ${ }^{13} \mathrm{C} /{ }^{15} \mathrm{~N}$ enriched spider dragline silk. A CP time of $1 \mathrm{~ms}$ was used, and the DD experiment was performed with a $1 \mathrm{sec}$ recycle delay. Adapted with permission from Ref. [174]. Copyright 2010 The Royal Society of Chemistry.

\section{ORGANIC ח-CONJUGATED POLYMERS}

Organic $\pi$-conjugated polymers are conducting materials that possess polymer backbones with extended $\pi$-conjugation. These semi-conductors have been used to fabricate electronic devices such as solar cells $[184,185]$, field effect transistors $[186,187]$ and light emitting diodes [184, 188, 189]. Organic polymers can easily be solution processed in a number of ways [190], an attractive fabrication property, since $\pi$-conjugated polymers spontaneously self-assemble into films from solution. Due to the high level of conjugation present in the polymers, $\pi-\pi$ interactions between chains impart some order to the selfassembled films. This usually results in a semi-crystalline system with regions of high and low order. This presents a number of challenges when films of these materials are studied via techniques that require high order, such as X-ray diffraction. On the other hand, many X-ray scattering methods can provide valuable information on the self-assembly of the molecules, such as the chain-to-chain and $\pi-\pi$ stacking lengths, and ordered stack orientations relative to the substrate plane [191]. However, determining the nature of the molecular packing is still quite difficult with these techniques, since they lack resolution at 
an atomic level. Due to the anisotropic nature of charge transport, molecular packing information is vital towards understanding the fundamentals of the effect of polymer molecular structure on optoelectronic properties [192]. To this end, solid-state NMR has the ability to provide critical information on the precise molecular packing. The following discussion will present some of the recent solid-state NMR work towards elucidating

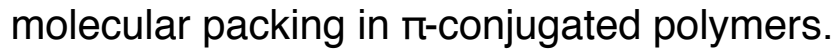

\subsection{Poly(3-hexyl thiophene) (P3HT): The reliable workhorse polymer}

P3HT is one of the most studied organic $\pi$-conjugated semi-crystalline polymers. Its relatively simple synthesis, facile processability, and high charge mobility all add to its appeal $[5,193]$. It consists of a polythiophenyl backbone with solubilizing alkyl side chains. P3HT's crystallinity is strongly dependent on the specific tacticity with which it is synthesized, the head-to-tail configuration being the most desirable for defect-free packing [194]. Dudenko et al. [195] provided a framework to reveal the local packing of the 3D crystalline phase of P3HT using solid-state NMR to complement X-ray diffraction (XRD) and molecular modeling. Two samples were used: a high molecular weight, low regioregular P3HT (P100) and a low molecular weight, high regioregular P3HT (P200). Using XRD, a monoclinic unit cell was determined for both along with the fraction of the crystalline and amorphous phases in the samples.

To extract molecular constraints of the crystallites, a combination of ${ }^{1} \mathrm{H}-{ }^{1} \mathrm{H}$ DQ-SQ NMR and ${ }^{13} \mathrm{C}\left\{{ }^{1} \mathrm{H}\right\}$ FSLG-HETCOR experiments were performed. Figure 16 shows the solid-state NMR spectra used in their study of annealed films of P100 and P200. The most interesting feature in the ${ }^{1} \mathrm{H}$ spectrum was observed in the aromatic region, where the thiophene 
signal is exclusively concentrated. A broad signal was observed resulting from the presence of two resonances centered at $6.9 \mathrm{ppm}$ and $6.0 \mathrm{ppm}$. In the liquid state, in which the polymer is not aggregated, only one signal near $6.9 \mathrm{ppm}(6.96 \mathrm{ppm})$ was observed. Hence, the peak at $6.9 \mathrm{ppm}$ was assigned to the amorphous phase of the polymer. Since this phase will be too unstructured to have closely apposed chains, and the closest intramolecular thiophene protons are about $6 \AA$ away, no autocorrelation should appear on the diagonal. This was confirmed by the ${ }^{1} \mathrm{H}-{ }^{1} \mathrm{H}$ DQ-SQ spectra (Figure $16 \mathrm{a}, \mathrm{c}$ ). Instead, an autocorrelation was only observed in the ${ }^{1} \mathrm{H}-{ }^{1} \mathrm{H}$ DQ-SQ spectrum for the $6 \mathrm{ppm}$ resonance. Coupled with the downfield shift of the peak relative to the liquid-state thiophene peak (a mark of $\pi-\pi$ interactions), the peak at $6.0 \mathrm{ppm}$ was assigned to the crystalline portion of the film. 

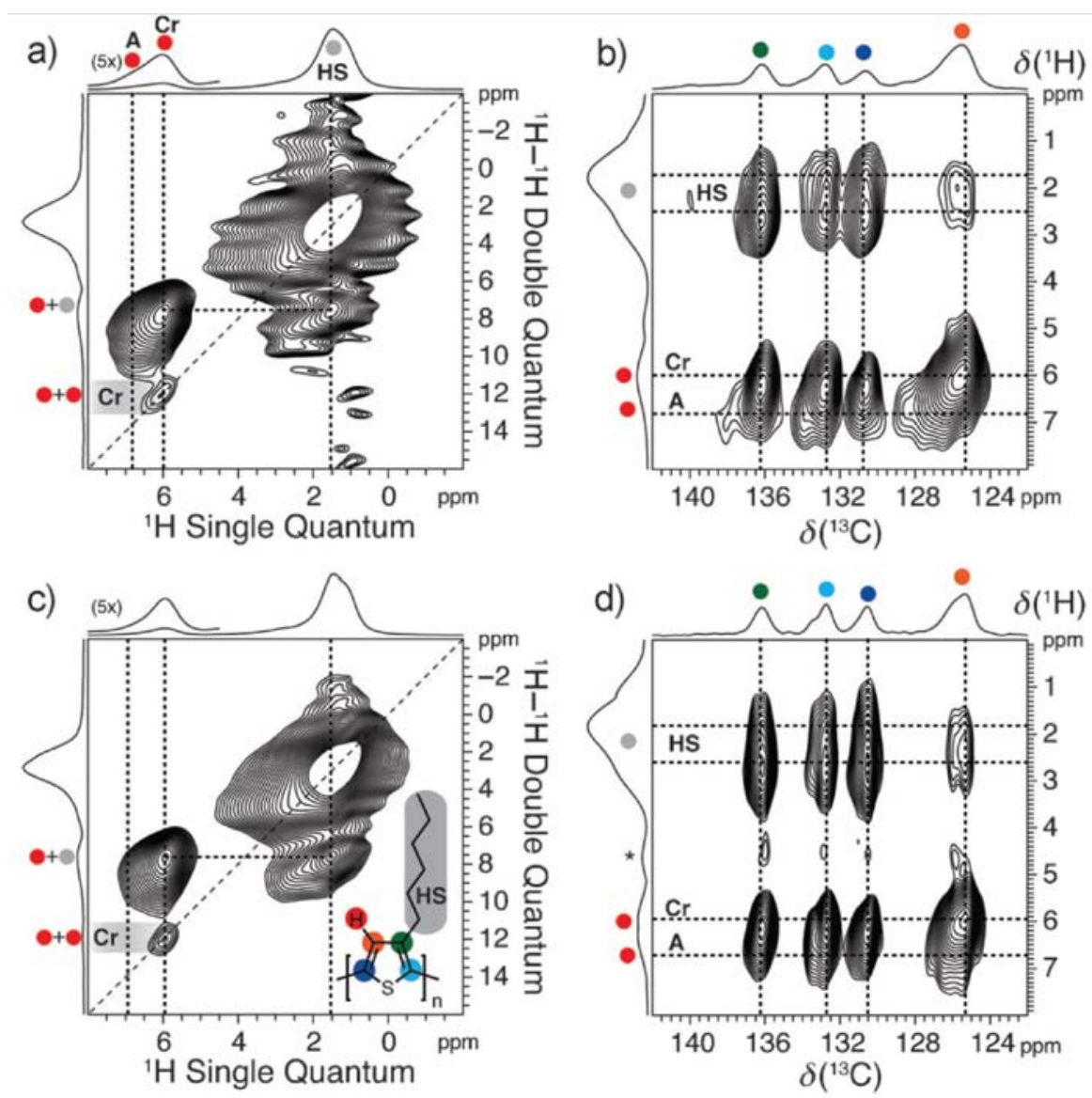

Figure 16. 2D ${ }^{1} \mathrm{H}-{ }^{1} \mathrm{H}$ DQ-SQ and ${ }^{13} \mathrm{C}\left\{{ }^{1} \mathrm{H}\right\}$ FSLG-HETCOR NMR spectra of $(\mathrm{a}, \mathrm{b}) \mathrm{P} 100$ and $(\mathrm{c}, \mathrm{d})$ P200, recorded at 20.0 T using spinning frequencies of 15.0 and $25.0 \mathrm{kHz}$, respectively. One rotor period of $\mathrm{BaBa}$ recoupling was used in $(\mathrm{a}, \mathrm{c})$, while a cross-polarization of 3.0 was used in $(b, d)$. A, $\mathbf{C r}$, and HS refer to the amorphous, crystalline, and hexyl side chain, respectively. The asterisk in (d) marks an artifact from the carrier frequency. Adapted with permission from Ref. [195]. Copyright 2012 Wiley.

The level of crystallinity was also observed in the ${ }^{13} \mathrm{C}\left\{{ }^{1} \mathrm{H}\right\}$ FSLG-HETCOR NMR spectra of both samples. As shown in Figure 16 ( $b$ and $d)$, the marked crystalline regions appeared as sharp resonances, while the amorphous regions appeared as broad shoulders. As expected, the higher regioregular polymer (P200) exhibited narrower resonances compared to its low regioregular (P100) counterpart, indicating a higher level of local order 
of its polymer chains. This observation corroborated with the level of crystallinity of both samples as determined from XRD and ${ }^{1} \mathrm{H}$ NMR.

Finally, the molecular constraints from the NMR experiments and the unit cell parameters from XRD were combined with Nucleus Independent Chemical Shift (NICS) calculations, to generate a model of the crystallite packing. Figure 17 shows the model that best fits with the NMR and XRD data, demonstrating the power of this strategy to gain insights into the packing of semi-crystalline polymers.

(a)

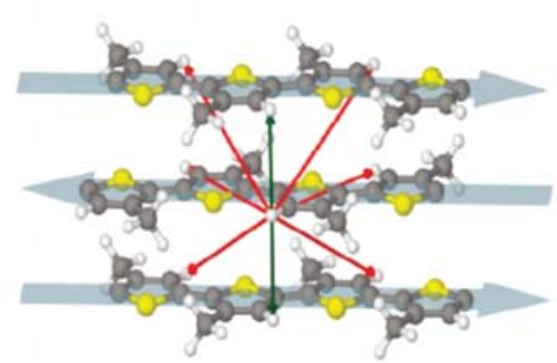

(b)
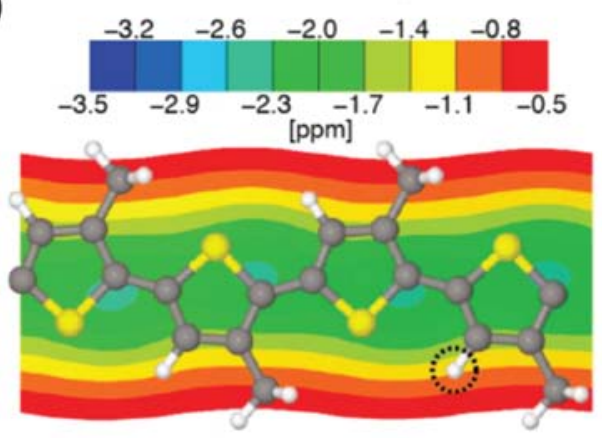

(c)

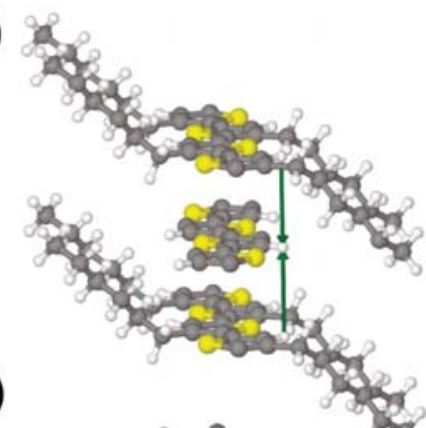

(d)

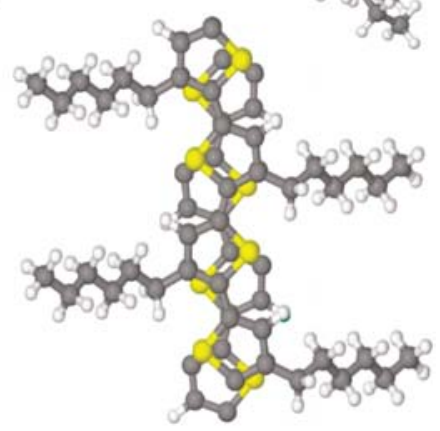

Figure 17. (a) Packing model and (b) its corresponding NICS map for P3HT in bulk samples after annealing that bests fit with the experimental. (c) Side and (d) top views of same model shown without the hexyl side chains for clarity. The green arrows indicate thiophene ${ }^{1} \mathrm{H}-{ }^{1} \mathrm{H}$ distances below $4 \AA$, while the red arrows indicate those that are above $4 \AA$. Adapted with permission from Ref. [195] Copyright 2012 Wiley. 


\subsection{Donor-acceptor m-conjugated polymers}

Polymers synthesized with a $\pi$-conjugated "donor-acceptor" (DA) architecture hold both electron rich (donor) and electron poor (acceptor) moieties within their monomeric unit. This molecular configuration offers many optoelectronic advantages, and has demonstrated the ability to facilitate very high charge mobilities [185]. Molecular packing within these polymers can be critically dependent on the DA pair. This was demonstrated by Warnan et al. [196] using two polymers synthesized with the same acceptor, a thienopyrroledione (TPD), but different donors, benzodithiophene (BDT) and benzodifuran (BDF), as shown in Figure 18. Grazing incidence X-ray scattering (GIXS) experiments of the neat polymers showed extensive $\pi-\pi$ interactions in PBDTTPD, as well as a preferential "face on" backbone orientation relative to the substrate. On the other hand, PBDFTP exhibited much lower $\pi-\pi$ aggregation, coupled with diffusely distributed backbone orientations relative to the substrate.

To shed light on the backbone packing of these two polymers, ${ }^{1} \mathrm{H}-{ }^{1} \mathrm{H}$ DQ-SQ NMR experiments were employed. As shown in Figure 18a, weakly resolved resonances between the alkyl protons of the two side chains and the backbone protons of BDTTPD could be observed in the ${ }^{1} \mathrm{H}-{ }^{1} \mathrm{H}$ DQ-SQ spectrum. For BDFTPD, on the other hand, many well-resolved correlations between the sidechains and BDF groups were apparent, as shown in Figure 18b. A weak intermolecular autocorrelation peak was also observed for BDFTPD (orange dotted circle in Figure 18b), signifying the presence of closely packed BDF chains. These taken in aggregate suggested that BDF units can adopt out of plane or twisted conformations relative to the TPD acceptor, while BDT adopts planar "syn" or "anti" conformations with TPD. While the twisted conformers of PBDFTP would weaken $\pi-\pi$ 
interactions, the backbone planarity exhibited by PBDTTP would tend to promote $\pi-\pi$ stacking, in line with the patterns observed in the GIXS measurements. Interestingly, while a molecular packing conformation difference would be expected to influence the optoelectronic properties of PBDTTPD and PBDFTPD, Mateker et al. [197] have recently shown that photo-oxidative stability could also be strongly affected by such conformations.
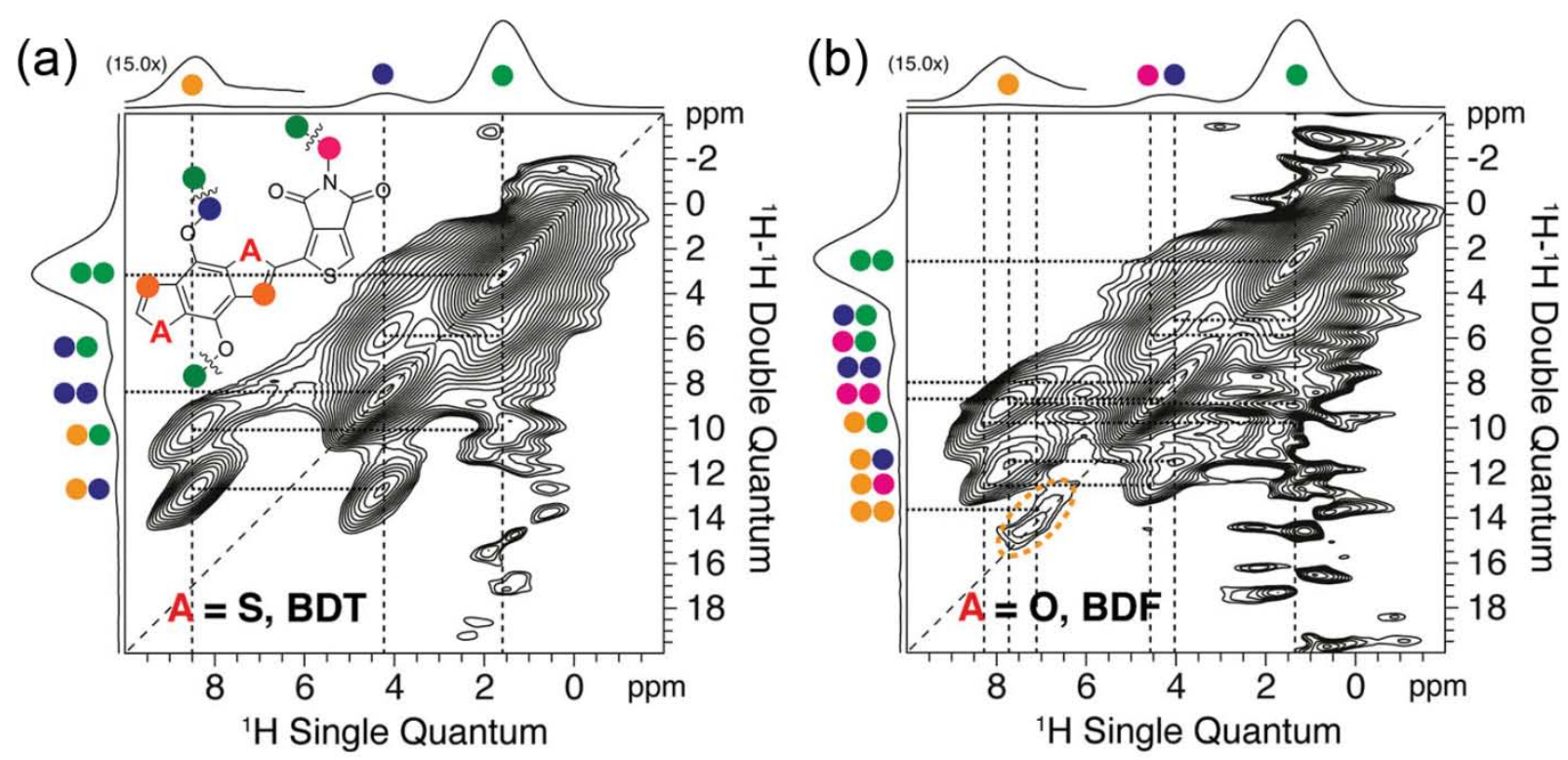

Figure 18. 2D ${ }^{1} \mathrm{H}-{ }^{1} \mathrm{H}$ DQ-SQ NMR spectrum of (a) BDTTPD and (b) BDFTP, recorded at $20.0 \mathrm{~T}$ using a spinning frequency of $29762 \mathrm{~Hz}$. One rotor period of $\mathrm{BaBa}$ recoupling was used for both materials. Adapted with permission from Ref. [196]. Copyright 2014 Wiley.

\section{ORGANIC SOLAR CELLS}

In organic solar cells, $\pi$-conjugated polymers occupy the energy harvesting layer, the socalled "active layer", along with an electron acceptor molecule (usually a fullerene analog). Absorption of photons by the polymer leads to the formation of an electron-hole pair, called an exciton, that is dissociated at the polymer:acceptor interface. One of the attractions of 
using organic polymers to construct solar cell devices is the solution co-processability of both the polymer and acceptor into a spontaneously formed mixed blend system known as a bulk heterojunction (BHJ) [185]. The BHJ architecture is composed of an interpenetrating network of polymer and acceptor, providing a large interfacial surface between the two. An optimal tailoring of the polymer:acceptor interface in terms of domain size and molecular interaction is critically important to fabricate highly efficient solar cells $[185,198,199]$. In the following sections, solid-state NMR efforts to address both of those aspects will be discussed.

\subsection{Domain size determination}

The domain size of polymer and acceptors in BHJs is a critically important parameter when fabricating high efficiency solar cells. The excitons generated by the absorption of sunlight have a limited diffusion length (up to $\sim 14 \mathrm{~nm}$ ) before they succumb to recombination [200-202]. Hence, the ideal interpenetrating BHJ network should have domains smaller than the exciton diffusion length for optimal dissociation. Domain sizes are usually characterized by transmission electron microscopy, atomic force microscopy and X-ray scattering methods $[191,203,204]$. Solid-state NMR could also be used for this purpose as well. Domain size determinations in polymer blends have been explored via solid-state NMR for quite some time [54, 205-207]. The obvious benefit of using solid-state NMR is the determination of domain sizes on the sub-nm range, which are difficult to examine using microscopic techniques. Furthermore, NMR allows for the interrogation of the whole BHJ blend rather than simply the blend-air interface, a limitation of surface contact techniques like AFM. 
Solid-state NMR techniques have been limited to examining thick films of BHJs for qualitative domain size determination due to the small quantity of film that results from popular thin film fabrication methods, like spin-coating. NMR relaxometery [208] and ${ }^{13} \mathrm{C}\left\{{ }^{1} \mathrm{H}\right\}$ FSLG-HETCOR (Figure 8) [124] experiments on thick films can give a sense of the domain size range in the film or whether extremely mixed domains $(<3-4 \AA)$ are present, respectively. In a unique attempt, Nieuwendaal et al. [55] reported a ${ }^{1} \mathrm{H}$ spin diffusion method to measure domain sizes in thin BHJ films of $\mathrm{P} 3 \mathrm{HT}$ : $[6,6]-$ phenyl- ${ }_{61}$-butyric acid methyl ester $\left(\mathrm{PC}_{61} \mathrm{BM}\right)$. As shown in Figure 19a, using a ${ }^{1} \mathrm{H}$ CRAMPS NMR probe, a high resolution spectrum could be obtained of a $\mathrm{P} 3 \mathrm{HT}: \mathrm{PC}_{61} \mathrm{BM}$ blend with relatively distinct contributions from the P3HT alkyl ( 2 ppm) and thiophene groups (6-7 ppm), and the $\mathrm{PC}_{61} \mathrm{BM}$ handle $(3 \mathrm{ppm})$ and phenyl groups (8 ppm). Furthermore, since thin films of P3HT:PC ${ }_{61} \mathrm{BM}$ blends $(\simeq 0.1 \mathrm{mg})$ were to be used for the experiments, background signal from exogenous protons were a concern. Hence, a specialized sample preparation protocol was developed in which deuterated reagents were exclusively used. 


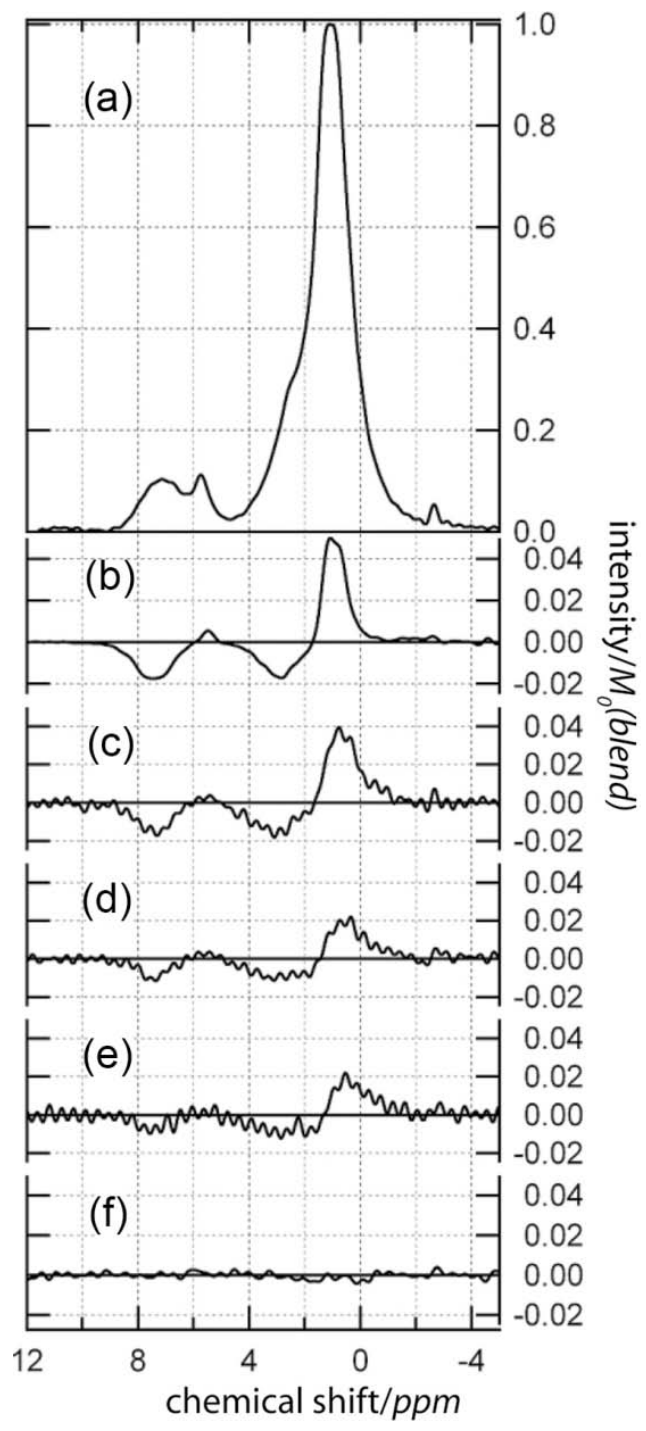

Figure 19. (a) ${ }^{1} \mathrm{H}$ CRAMPS NMR spectrum of the slow-spin unannealed thin film $\mathrm{P} 3 \mathrm{HT}: \mathrm{PCBM}$ blend. Also, ${ }^{1} \mathrm{H}$ CRAMPS NMR spin diffusion spectrum representing a physical P3HT-PCBM (5050 by mass) mixture at $t_{m}=2 \mathrm{~ms}(b)$, and that of the slow-spin unannealed thin film blend for $t_{m}=$ (c) $2 \mathrm{~ms}$, (d) $30 \mathrm{~ms}$, (e) $60 \mathrm{~ms}$, and (f) $240 \mathrm{~ms}$. All experiments were performed at $7.0 \mathrm{~T}$ using a spinning frequency of $2525 \mathrm{~Hz}$. Adapted with permission from Ref. [55]. Copyright 2012 Wiley.

A ${ }^{1} \mathrm{H}$ spin diffusion approach was taken in which a chemical shift based gradient between the $\mathrm{P} 3 \mathrm{HT}$ and $\mathrm{PC}_{61} \mathrm{BM}$ was established, and the magnitude of that gradient was measured as a function of the mixing time, $t_{m}$. The chemical shift gradient was established using an MREV8 preparation step [209], which essentially has the effect of multiplying the CRAMPS spectrum with a sine wave. Even with some spectral overlap of signals from 
$\mathrm{P} 3 \mathrm{HT}$ and $\mathrm{PC}_{61} \mathrm{BM}$, the majority of the $\mathrm{P} 3 \mathrm{HT}$ signal assumes a positive integral while that of PCBM exhibits a negative integral, as shown in Figure 19b. Also, Figure 19 (c-f) shows the change in spectrum as a function of the mixing time. Note that these spectra show the effects of only intermolecular spin exchange, since intramolecular spin equlibriation has been reached before $t_{m}=2$ ms. Figure 20 shows the ${ }^{1} \mathrm{H}$ spin diffusion curves of P3HT:PC ${ }_{61} B M$ films that were spun-cast using different processing conditions. The magnitude of the magnetization gradient $(\Delta M)$ was quantified by comparison of the experimental intensity to that of a physical mixture of similar $\mathrm{P} 3 \mathrm{HT}$ and $\mathrm{PC}_{61} \mathrm{BM}$ compositions. It was normalized so that $\Delta \mathrm{M}=1$ at $\mathrm{t}_{\mathrm{m}}=0$, and was appropriately corrected to remove $T_{1}$ effects.

An analysis framework was developed to fit the ${ }^{1} \mathrm{H}$ spin diffusion data, using Equation 1 as a basis:

$$
x=\frac{\epsilon}{f_{b}} \sqrt{\frac{4 D_{\text {eff }}}{\pi}} t_{1 / 2}
$$

where $x$ is the domain in $\mathrm{nm}, \epsilon$ is either 1 (lamella) or 2 (rods-in-a-hexagonal-matrix), $f_{b}$ is the volume fraction of the major phase, $b$, and $D_{\text {eff }}$ is the experimentally determined spin diffusion coefficient $\left(0.4 \mathrm{~nm}^{2} \mathrm{~ms}^{-1}\right)$. The lamellar and rods-in-a-hexagonal-matrix models were both considered since not one is complete enough to describe the interpenetrating, complex morphology of the BHJ. Since the initial points of the ${ }^{1} \mathrm{H}$ spin diffusion curves were not linear (an indication of the presence of many different domain sizes), an analysis method was used in which a distribution of domain sizes was considered. Using appropriate domain size dependencies for the lamellar and rods-in-a-hexagonal-matrix models, the ${ }^{1} \mathrm{H}$ spin diffusion data fit was a result of the linear combination of contributions from eight different $\mathrm{PC}_{61} \mathrm{BM}$ domain sizes. These were arbitrarily chosen and ranged from 
very small $(1.5 \mathrm{~nm})$ to very large (ie. too large to experience any appreciable decay). Figure 20 shows an example of a linear least-squares fit of the ${ }^{1} \mathrm{H}$ spin diffusion data to the lamellar morphology model.

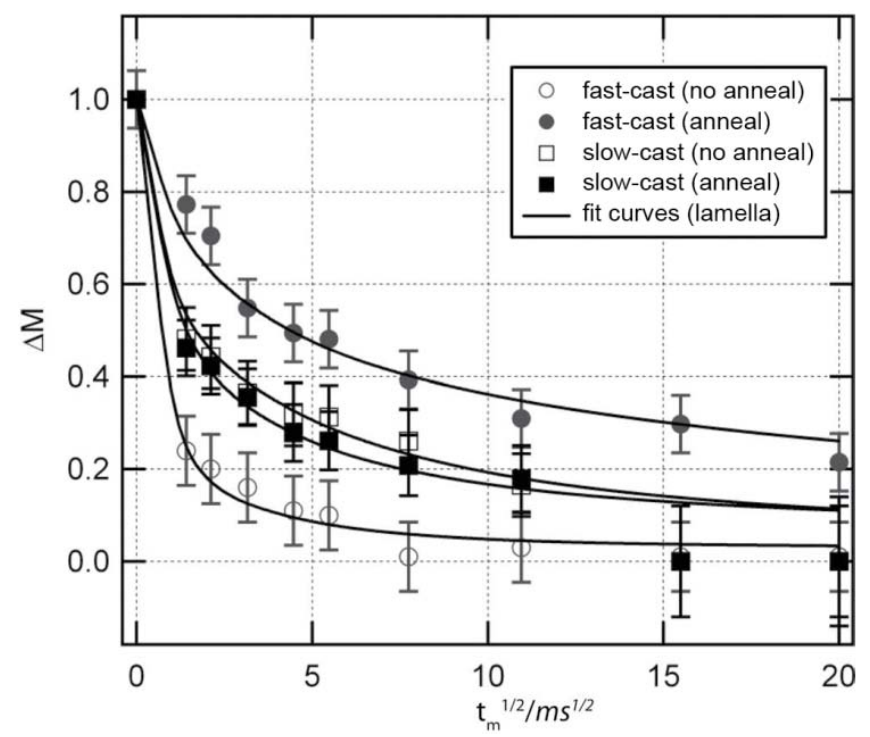

Figure 20. ${ }^{1} \mathrm{H}$ spin diffusion NMR curves of P3HT:PCBM thin films spun-cast using the stated processing recipes. The curves shown were fit using the lamellar model. Adapted with permission from Ref. [55]. Copyright 2012 Wiley.

Figure 21 shows the domain size distributions of the $\mathrm{P} 3 \mathrm{HT}: \mathrm{PC} \mathrm{C}_{61} \mathrm{BM}$ thin films prepared using different preparation protocols, via both the lamellar and rods-in-a-hexagonal-matrix models. It is worth noting that both models show similar qualitative trends in domain size distributions. When comparing the domain sizes resulting from the processing techniques, notable differences are seen between the fast-cast non-annealed and annealed cases, as well as the fast-cast and slow-cast cases. The fast-cast non-annealed domain distribution is heavily shifted towards the smaller $(<10 \mathrm{~nm})$ sizes, while the annealed samples exhibit a much broader distribution of domain sizes. Meanwhile, the slow-cast films contain domain sizes that are intermediate of the non-annealed and annealed cases of the fast-cast films. Concurrently, the slow cast films contain much smaller numbers of very large $(>100 \mathrm{~nm})$ 
domains. Photoluminescence measurements correlate well with the domain sizes determined with this method. The fast-cast as-cast films show very low PL intensity, indicative of very good mixing (i.e. small domains), while the relatively broadly distributed films of the other preparations accordingly exhibit a higher PL intensity.
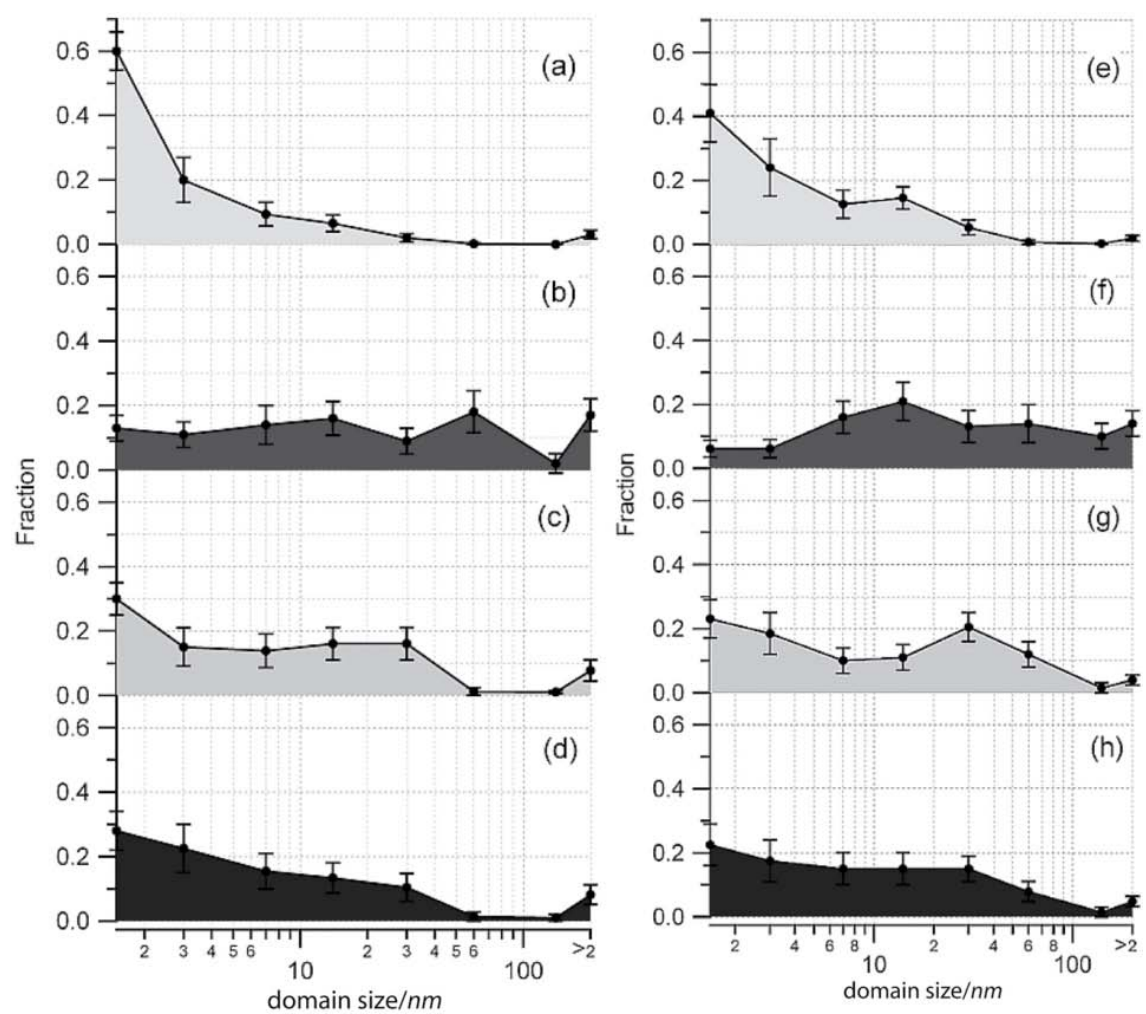

Figure 21. Domain size distributions estimated for thin P3HT:PCBM films assuming both (a-d) the lamellar, and (e-h) rods-in-a-hexagonal-matrix morphologies. Fast-cast, $(a, e)$ non-annealed and (b, f) annealed, are shown against slow-cast, $(\mathrm{c}, \mathrm{g})$ non-annealed and $(\mathrm{d}, \mathrm{h})$ annealed preparations. The $x$-axis is scaled logarithmically. Mass fractions are shown on the $y$-axis. Adapted with permission from Ref. [55]. Copyright 2012 Wiley.

The domain size distribution can also aid in clarifying the reasons behind some of the trends in efficiency of the films in devices. For example, the fast-cast, non-annealed device exhibited the lowest efficiency amongst the processing conditions used by the authors. Since $>80 \%$ of the domains are near the exciton diffusion length $(<10 \mathrm{~nm})$, poor efficiency would have to be attributed to low mobility or excess recombination rather than lack of 
interfacial contact. Meanwhile, the slow-cast, non-annealed device yielded the highest efficiency. This film exhibited a relatively good spatial homogeneity in the moderate range of domain sizes (Figure $21 \mathrm{c}$ and $\mathrm{g}$ ), indicating a way to form an efficient percolative charge transport network.

Due to the very heterogenous nature of the $\mathrm{BHJ}$, this method will remain more of a qualitative measure of the range of domains present in thin films rather than a quantitative one. Also, due to the limited spectral range of ${ }^{1} \mathrm{H}$ and broad peaks resulting from homonuclear dipolar coupling, the technique may find limited appeal with more complex structures, like DA polymers, which usually contain many more and types of protons than $\mathrm{PC}_{61} \mathrm{BM}$, leading to severe spectral overlap. However, under appropriate conditions, it remains quite powerful in exploring the existence of very small domains $(<10 \mathrm{~nm})$, which is quite difficult via other techniques.

\subsection{Polymer/acceptor interactions in bulk heterojunctions}

At the polymer/acceptor interface, the arrangement of the polymer and acceptor relative to each other can have a profound effect on charge dissociation [198, 199, 210]. The heterojunction interface complexity effectively limits the characterization techniques to those capable of providing angstrom level resolution. As mentioned before, solid-state NMR is one of the few techniques that fit this requirement.

Contributions to the study of poly-(2,5-bis(3-alkylthiophen-2-yl)thieno[3,2-b]thiophene) (PBTTT):PCBM BHJs are good examples on how solid-state NMR techniques can provide insight into the donor:acceptor interface. PBTTT-R (Figure 22) is a semicrystalline polymer which can self-assemble into large crystalline domains, exhibiting very large field-effect 
mobilities [211]. It can be synthesized with a variety of solubilizing aliphatic side chains (R). $\mathrm{PC}_{71} \mathrm{BM}$ is known to intercalate between the side chains of PBTTT, altering performance of the BHJ relative to non-intercalated blends [212]. Miller et al. [213] studied the $\mathrm{BHJ}$ structure using $\mathrm{XRD}, \mathrm{MD}$ simulations, IR spectroscopy and 2D solid-state NMR to detail the BHJ blend at the molecular level. Solid-state NMR was employed to complement the other methods, and also provided insight into the disordered moieties of the blend, an aspect inaccessible to most techniques.

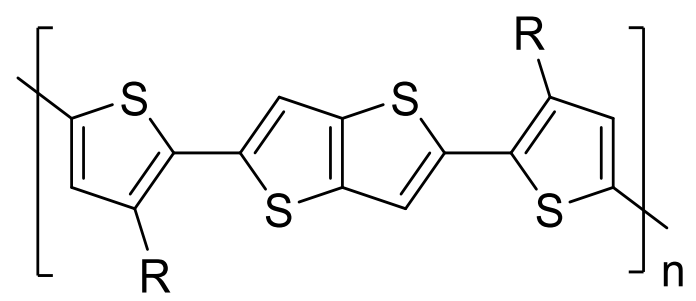

Figure 22. Chemical structure of PBTTT-R. $R$ is the alkyl solubilizing side chain. Only results from $\mathrm{R}=\mathrm{C} 16$ (hexadecanyl-) and $\mathrm{R}=\mathrm{C} 14$ (tetradecanyl-) are presented here.

The 2D solid-state NMR contribution primarily consisted of ${ }^{13} \mathrm{C}\left\{{ }^{1} \mathrm{H}\right\}$ e-DUMBO-HETCOR experiments, with the aim to correlate intermolecular interactions between PBTTT-C16 and the intercalated $\mathrm{PC}_{71} \mathrm{BM}$. Figure 23a shows the ${ }^{13} \mathrm{C}\left\{{ }^{1} \mathrm{H}\right\}$ e-DUMBO HETCOR spectrum of the drop-casted PBTTT-C16:PCBM blend (1:1). Quite a number of signals from the $\mathrm{PC}_{71} \mathrm{BM}$ (in blue) were found to be correlated with those from the PBTTT-C16 (in orange). In particular, $\mathrm{PC}_{71} \mathrm{BM}{ }^{13} \mathrm{C}$ peaks from the carbonyl (172 ppm), phenyl ring (129 ppm) and the cyclopropane anchor (73 ppm) were found to be correlated with the ${ }^{1} \mathrm{H}$ signals at 1.4 ppm from the C16 chain of PBTTT-C16. These correlations established the close proximity of the $\mathrm{PC}_{71} \mathrm{BM}$ handle to the sidechains of PBTTT. Also, ${ }^{13} \mathrm{C}$ signals from the $\mathrm{C}_{71}$ fullerene groups (147-152 ppm) show correlations with ${ }^{1} \mathrm{H}$ signals from the aliphatic side chains (1.4 ppm) and aromatic backbone (7.2 and $8.7 \mathrm{ppm}$ ) of PBTTT-C16, indicating the close 
molecular interactions of the $\mathrm{PC}_{71} \mathrm{BM}$ cage with the polymer. Furthermore, ${ }^{13} \mathrm{C}$ signals from the PBTTT-C16 backbone moieties (131, 135 and $140 \mathrm{ppm}$ ) correlated with the ${ }^{1} \mathrm{H}$ signal from the $\mathrm{PC}_{71} \mathrm{BM}$ phenyl group (7.9 ppm), suggesting the existence of an orientation where the $\mathrm{PC}_{71} \mathrm{BM}$ handle points towards the PBTTT-C16 backbone. This is especially interesting in the light of the space-filling structure of the PBTTT-C14:PC71BM bimolecular crystal (Figure 23b), determined from XRD and ${ }^{1} \mathrm{H}-{ }^{1} \mathrm{H}$ DQ-SQ NMR constraints. Though much of the HETCOR data bears out the details of the simulated unit cell, it differs in that the unit cell suggests that the $\mathrm{PC}_{71} \mathrm{BM}$ handles are at a $180^{\circ}$ away from the polymer backbone. The fact that the 2D HETCOR suggests the existence of an orientation that is opposite to this, shows the ability of the technique to provide direct proof of the intrinsic local structural disorder that is present in the system. The authors suggest that this orientational disorder may result in disrupting the 1D fullerene channels, and thus, decrease the electron transport efficiency in these pathways. 

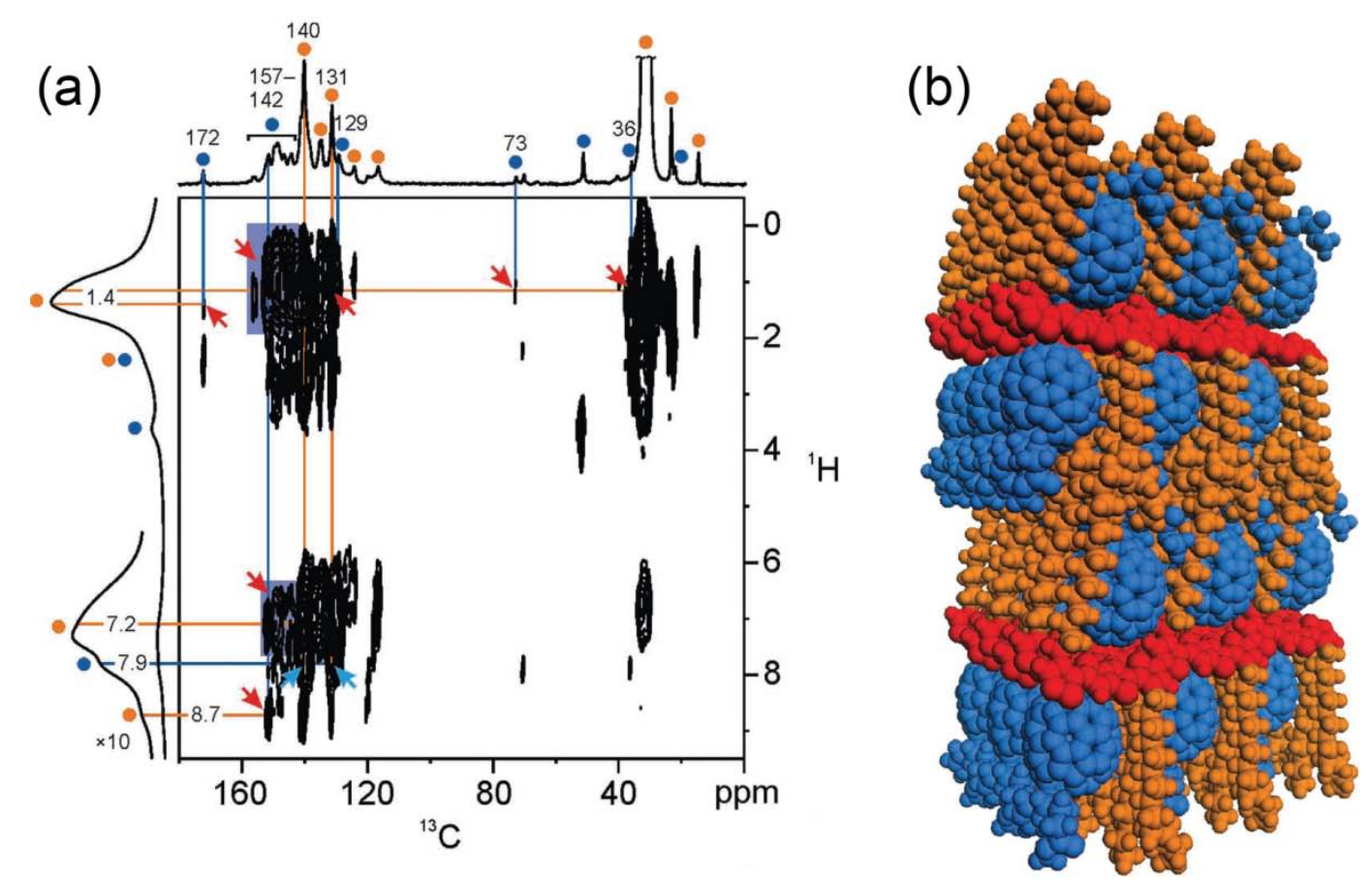

Figure 23. (a) 2D ${ }^{13} \mathrm{C}\left\{{ }^{1} \mathrm{H}\right\}$ e-DUMBO-HETCOR NMR spectrum of PBTTT-C16:PC ${ }_{71} \mathrm{BM}(1: 1)$. The top axis is a $1 \mathrm{D}^{13} \mathrm{C}\left\{{ }^{1} \mathrm{H}\right\} \mathrm{CP} / \mathrm{MAS}$ spectrum and the left axis is a $1 \mathrm{D}$ single-pulse ${ }^{1} \mathrm{H}$ MAS spectrum of the same sample. Spectral contributions from PBTTT-C16 and $\mathrm{PC}_{71} \mathrm{BM}$ are indicated by orange and blue circles, respectively. The red arrows mark correlations between ${ }^{13} \mathrm{C}$ moieties of $\mathrm{PC}_{71} \mathrm{BM}$ and ${ }^{1} \mathrm{H}$ moieties of PBTTT-C16. The light-blue arrows indicate the correlations between the ${ }^{13} \mathrm{C}$ aromatic groups of PBTTT-C16 and the ${ }^{1} \mathrm{H}$ moieties of the $\mathrm{PC}_{71} \mathrm{BM}$ handle. The spectrum was recorded at $11.7 \mathrm{~T}$ using a spinning frequency of $12500 \mathrm{~Hz}$. A cross-polarization time of 2 ms was used. (b) A space-filling structure of the PBTTT-C14:PC $\mathrm{P}_{71} \mathrm{BM}$ bimolecular crystal based on the simulated unit cell. The PBTTT-C14 backbone is shown in red, its aliphatic chains in orange and the $\mathrm{PC}_{71} \mathrm{BM}$ in blue. Adapted with permission from Ref. [213]. Copyright 2012 Wiley.

In this study, the high crystallinity of the PBTTT-C16:PC ${ }_{71} \mathrm{BM}$ blend as well as a low spectral overlap between the ${ }^{13} \mathrm{C}$ and ${ }^{1} \mathrm{H}$ resonances from both units resulted in well resolved 2D solid-state NMR spectra. However, polymers with low crystallinity and more complex structures that have a spread of chemical shifts in both the aliphatic and aromatic regions, might not yield similar well-resolved spectra. For those cases, more selective NMR experiments, like the 2D MELODI-HETCOR presented in Section 3.9, would be more appropriate. 


\section{GRAPHENE AND RELATED MATERIALS}

\subsection{Graphene}

For several decades, graphene has been used as an exciting theoretical model to describe various properties of carbon-based materials, and as a condensed matter analogue of (2+1)-dimensional quantum electrodynamics in physics [214]. In 2004, Novoselov et al. $[215,216]$ turned graphene into a reality. Graphene is a two-dimensional monolayer with the carbons being arranged in a honeycomb lattice. It is envisioned to have great potential for a variety of applications, such as in energy-storage devices [217-219] and electronic devices [220-223]. Solid-state NMR spectroscopy provides an important characterization tool for graphenes in which the number of unpaired delocalized electrons is kept to a minimum $[224,225]$.

A spectrum of exfoliated graphene is expected to exhibit broad peaks due to very short relaxation times originating from the strong couplings between the nuclei and the unpaired electrons. 1D ${ }^{13} \mathrm{C}$ MAS NMR spectra acquired on exfoliated graphene and graphene oxide [226] at 16.4 T using $30 \mathrm{kHz}$ MAS are displayed in Figure 24. Indeed, the signals assigned to $\mathrm{sp}^{2}$-hybridized ${ }^{13} \mathrm{C}$ nuclei at $122 \mathrm{ppm}$ are very broad. The signals at $70-60 \mathrm{ppm}$ for graphene oxide are assigned to $\mathrm{sp}^{3}$-hybridized ${ }^{13} \mathrm{C}$ nuclei attached to oxygen. The overall experimental time to obtain a reasonable signal-to-noise ratio for exfoliated graphene was approximately three days. In case of exfoliated graphene, a partially filled rotor was used as eddy current heating was induced in the sample due to MAS. 


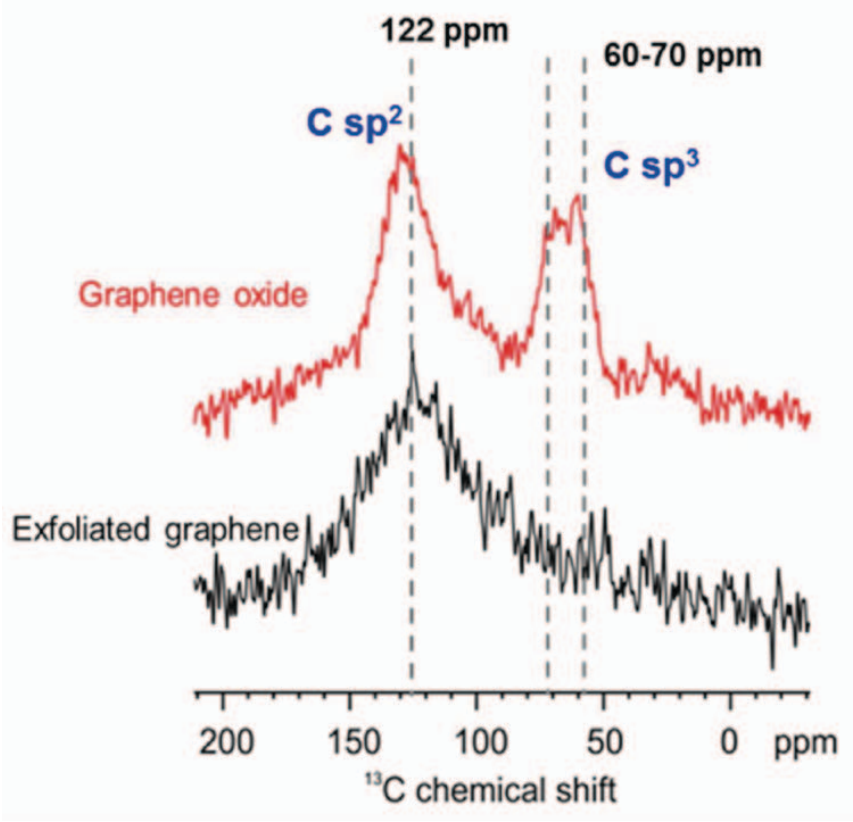

回

Figure 24. 1D ${ }^{13} \mathrm{C}$ MAS NMR spectra of exfoliated graphene (black) and graphene oxide (red), respectively. The spectra were recorded at $16.4 \mathrm{~T}$ using $30 \mathrm{kHz}$ MAS. In exfoliated graphene and graphene oxide, the broad signals at ca. $122 \mathrm{ppm}$ are assigned $\mathrm{sp}^{2}$-hybridized ${ }^{13} \mathrm{C}$ nuclei. In graphene oxide, signals ascribed to $\mathrm{sp}^{3}$-hybridized ${ }^{13} \mathrm{C}$ nuclei attached to oxygen are observed between 70 and 60 ppm. Adapted with permission from Ref. [226]. Copyright 2014 American Chemical Society.

?

\subsection{Graphite oxide}

Turning to graphite oxide, solid-state NMR spectroscopy has been used to provide important structural insights [227]. To overcome the fact that ${ }^{13} \mathrm{C}-{ }^{13} \mathrm{C}$ bonds exist with a probability of $0.01 \%$ in natural samples, Cai et al. prepared ${ }^{13} \mathrm{C}$-labelled graphite oxide, making the sample amenable to finite-pulse radio frequency-driven recoupling (fpRFDR) $[228]{ }^{13} \mathrm{C}-{ }^{13} \mathrm{C}$ correlation spectroscopy. Figure $25 \mathrm{~A}$ shows the $1 \mathrm{D}{ }^{13} \mathrm{C}$ MAS NMR spectrum which assigns the signal at $129 \mathrm{ppm}$ to $\mathrm{sp}^{2}$-hybridized ${ }^{13} \mathrm{C}$ nuclei, the signal at $70 \mathrm{ppm}$ to ${ }^{13} \mathrm{C}-\mathrm{OH}$ nuclei and the signal $60 \mathrm{ppm}$ to epoxide ${ }^{13} \mathrm{C}$ nuclei. In addition, three smaller signals are seen at 193 ppm, 169 ppm and 101 ppm, respectively. 


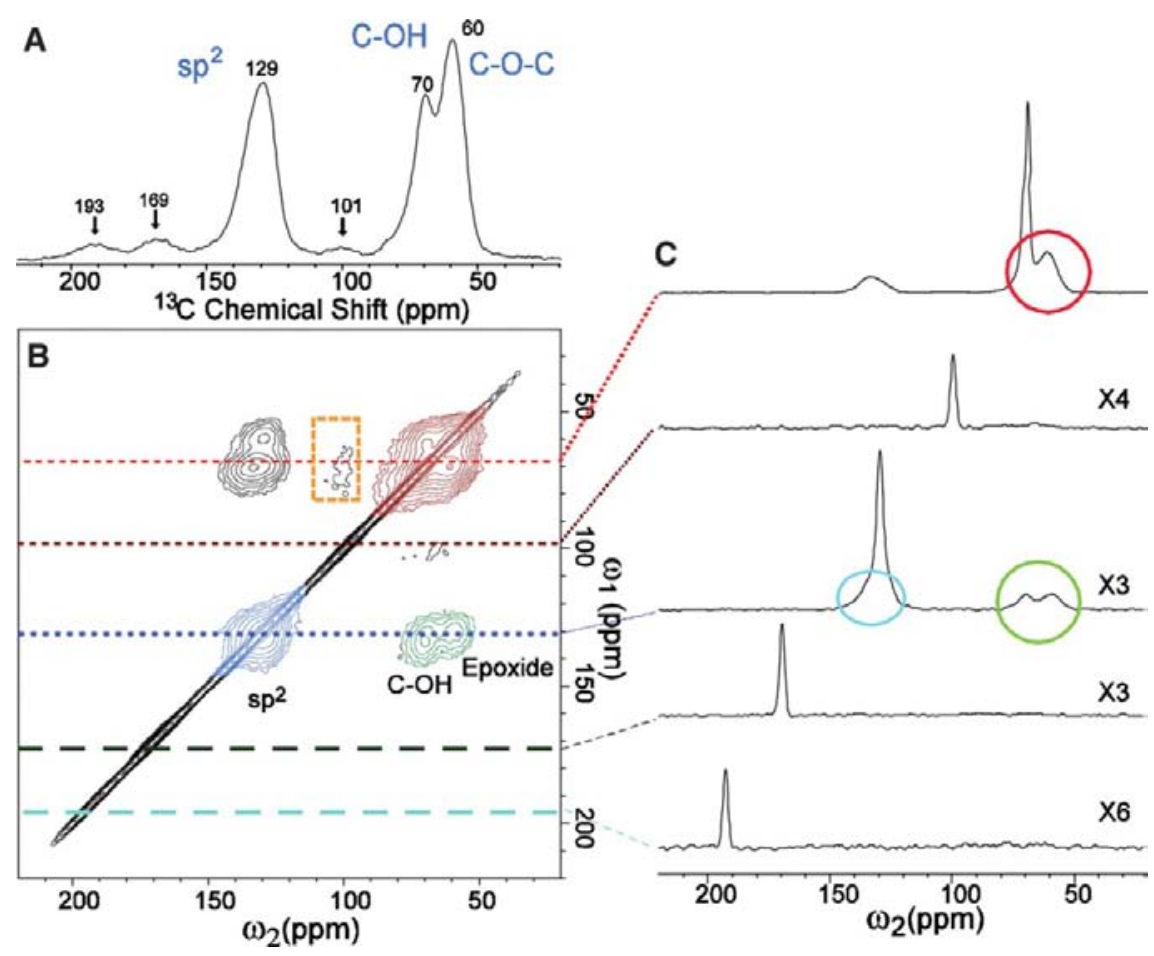

Figure 25. (A) The $1 \mathrm{D}{ }^{13} \mathrm{C}$ MAS spectrum of ${ }^{13} \mathrm{C}$-labelled graphite oxide. The three major signals at $129 \mathrm{ppm}, 70 \mathrm{ppm}$ and $60 \mathrm{ppm}$ are assigned to $\mathrm{sp}^{2}$-hybridized ${ }^{13} \mathrm{C}$ nuclei, ${ }^{13} \mathrm{C}-\mathrm{OH}$ nuclei and to epoxide ${ }^{13} \mathrm{C}$ nuclei, respectively. (B) The $2 \mathrm{D}{ }^{13} \mathrm{C}-{ }^{13} \mathrm{C}$ fpRFDR correlation spectrum used $1.6 \mathrm{~ms}$ mixing time to identify mainly one- or two-bond correlations. (C) Slices are extracted to highlight the important ${ }^{13} \mathrm{C}-{ }^{13} \mathrm{C}$ correlations. Data was acquired $9.4 \mathrm{~T}$ using $20 \mathrm{kHz}$ MAS. Adapted with permission from Ref. [227]. Copyright 2008 Science.

The fpRFDR ${ }^{13} \mathrm{C}-{ }^{13} \mathrm{C}$ correlation spectrum can be seen in Figure $25 \mathrm{~B}$. The cross correlations highlighted in green in Figure 25B signify the contact between $\mathrm{sp}^{2}$-hybridized ${ }^{13} \mathrm{C}$ nuclei and ${ }^{13} \mathrm{C}-\mathrm{OH}$ nuclei $\left(\omega_{1}: 133 \mathrm{ppm} ; \omega_{2}: 70 \mathrm{ppm}\right)$ and the contact between $\mathrm{sp}^{2}-$ hybridized ${ }^{13} \mathrm{C}$ nuclei and epoxide ${ }^{13} \mathrm{C}$ nuclei $\left(\omega_{1}: 130 \mathrm{ppm} ; \omega_{2}: 59 \mathrm{ppm}\right)$. The contact between ${ }^{13} \mathrm{C}-\mathrm{OH}$ nuclei and epoxide ${ }^{13} \mathrm{C}$ nuclei is highlighted in red in Figure 25B. The blue correlations indicate the internal contact between $\mathrm{sp}^{2}$-hybridized ${ }^{13} \mathrm{C}$ nuclei. Of the minor signals, only the $101 \mathrm{ppm}$ nuclei show weak correlations (highlighted in orange) with the ${ }^{13} \mathrm{C}-\mathrm{OH}$ nuclei, and to a lesser extent, with epoxide ${ }^{13} \mathrm{C}$ nuclei. The ${ }^{13} \mathrm{C}=\mathrm{O}$ nuclei assigned to the resonance frequencies at $193 \mathrm{ppm}\left(\mathrm{R}-{ }^{13} \mathrm{CO}-\mathrm{R} 1\right)$ and $169 \mathrm{ppm}\left({ }^{13} \mathrm{COOH}\right)$ do not 
exhibit any correlations which indicates that these nuclei are spatially separated from the rest. The findings of the study support the two structural models, the Dékány model [229] and the Lerf-Klinowski model [230], out of six models [229] of graphite oxide.

In another study on this material, ${ }^{13} \mathrm{C}$-labelled graphite oxide was reacted with ${ }^{15} \mathrm{~N}$ laballed hydrazine $\left({ }^{15} \mathrm{NH}_{2}{ }^{15} \mathrm{NH}_{2}\right)$ to produce a reduced ${ }^{13} \mathrm{C}$ - and ${ }^{15} \mathrm{~N}$-labelled version of the molecule [231]. Figure 26a displays the $1 \mathrm{D}{ }^{13} \mathrm{C}$ MAS spectrum. Compared to Figure 25A, the spectrum in Figure 26a shows a shift in the main signal to ca. $118 \mathrm{ppm}$ which signifies a larger $\mathrm{sp}^{2}$-hybridized conjugation. The signals at ca. $70 \mathrm{ppm}$ and $60 \mathrm{ppm}$ assigned previously to ${ }^{13} \mathrm{C}-\mathrm{OH}$ nuclei and epoxide ${ }^{13} \mathrm{C}$ nuclei, respectively, are clearly suppressed showing the effects of reduction by hydrazine. The peak at $193 \mathrm{ppm}$ ascribed to ketone groups in graphite oxide is likewise much weaker, indicating that chemical changes had occurred on the edges. Some of these changes are illustrated in Figure 26d.

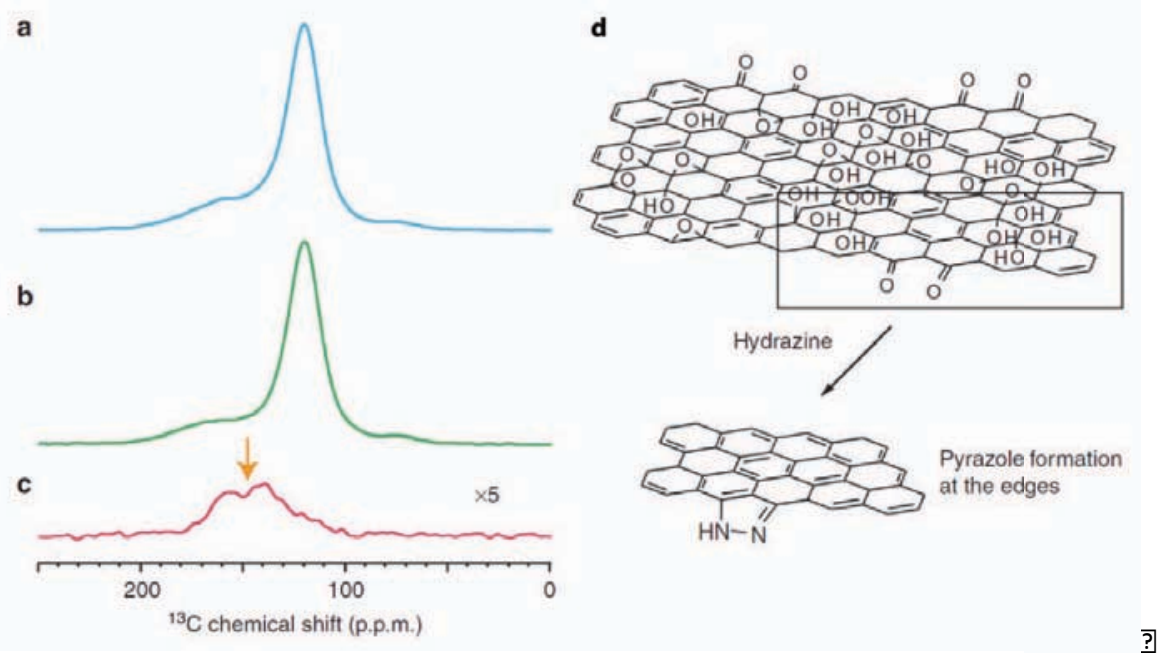

Figure 26. $1 \mathrm{D}{ }^{13} \mathrm{C}$ NMR MAS spectra of ${ }^{13} \mathrm{C}$ - and ${ }^{15} \mathrm{~N}$-labelled reduced graphite oxide (a) not subjected and (b) subjected to $1.6 \mathrm{~ms}{ }^{13} \mathrm{C}-{ }^{15} \mathrm{~N}$ REDOR dephasing and (c) the difference of the two. The study suggests that the double signal centered at ca. $150 \mathrm{ppm}$ is ascribed to pyrazole groups (aromatic five-membered rings with two adjacent $\mathrm{N}$ atoms). An illustration of the formation of pyrazole moieties with the $\mathrm{COOH}$ groups omitted for clarity (d). The data was acquired at $9.4 \mathrm{~T}$ using $20 \mathrm{kHz}$ MAS. Adapted with permission from Ref. [231]. Copyright 2012 Nature Publishing Group. 
To gain further insight into the signals at ca. $157 \mathrm{ppm}$ and $140 \mathrm{ppm}$, which are overlapped by the largest signal in a normal single-pulse experiment, ${ }^{13} \mathrm{C}-{ }^{15} \mathrm{~N}$ dipolar REDOR dephasing [44] was applied. The spectrum subjected to dipolar dephasing was subtracted from the reference spectrum, thereby revealing those ${ }^{13} \mathrm{C}$ nuclei that are at one bond or, at the most, two bonds away from ${ }^{15} \mathrm{~N}$ nuclei, cf. Figure $26 \mathrm{c}$. The resonance frequencies of the ${ }^{13} \mathrm{C}$ nuclei can in principle be related to a number of different chemical structures, however, taken together with the information from ${ }^{15} \mathrm{~N}$ NMR spectroscopy, showing a broad signal at ca. 190 ppm, the study suggests that aromatic pyrazole groups are established at edges after treatment with hydrazine.

\subsection{Graphene nanoribbons}

Graphene nanoribbons (GNRs) are promising candidates for the next-generation semiconductor material [232-235]. The width and the edge of GNRs define finite bandgap properties [232, 235], and hence, attract much attention from synthetic chemists. Osella et al. [236] have used time-dependent density functional theory calculations to suggest chemical designs for GNRs that are applicable to future OPV development. All computertested GNRs possess a finite energy bandgap and exhibit lower electron affinity than $\mathrm{C}_{60}$. In addition, the armchair GNRs have stronger absorption in a broader and red-shifted region of the solar spectrum than one of the workhorses of OPV, P3HT, (see Section 6.1). Compared to P3HT, all GNRs demonstrate lower HOMOs and a smaller singlet-triplet energy gap, which should facilitate larger open circuit voltage. Apart from the optical aspects of OPV materials, morphology also plays an important role governing the final properties of the material. In relation to production, the GNRs should also be soluble in organic solvents and should be able to form films. 
Recently, bottom-up procedures have been applied to synthesize extended (in some cases longer than $200 \mathrm{~nm}$ ) GNRs [237, 238]. The starting material is polyphenylene with very large molecular weights prepared by $A B$-type Diels-Alder polymerization. Intramolecular oxidative cyclohydrogenation turns the precursors into GNRs. As an example of the importance of the width, GNRs [238] with an extended width of ca. $2 \mathrm{~nm}$ showed a bandgap of ca. $1.2 \mathrm{eV}$ whereas GNRs [237] with a limited width of ca. $1 \mathrm{~nm}$ showed a bandgap of ca. $1.9 \mathrm{ev}$. A schematic representation of the latter GNR can be seen in Figure 27f. As ${ }^{1} \mathrm{H}$ MAS NMR spectroscopy is very sensitive to the local environment, the technique can used to probe the chemical differences between the precursor in the solid-state and the GNR sample. In Figure 27b, the $1 \mathrm{D}{ }^{1} \mathrm{H}$ MAS NMR spectrum of the precursor is shown. The relatively narrow resonances of the precursor, which shares some similarity with the liquid-state spectrum in Figure $27 a$, indicates a flexible structure. On the other hand, the 1D ${ }^{1} \mathrm{H}$ MAS NMR spectrum of GNR in Figure 27c exhibits significantly broadened and shifted signals. These features indicate the presence of an extended inhomogeneous packed $\pi$-conjugated system. The opposite shift of the aromatic and aliphatic signals might be explained by the aromatic/anti-aromatic ring current effects originating from the stacking of the GNR. 

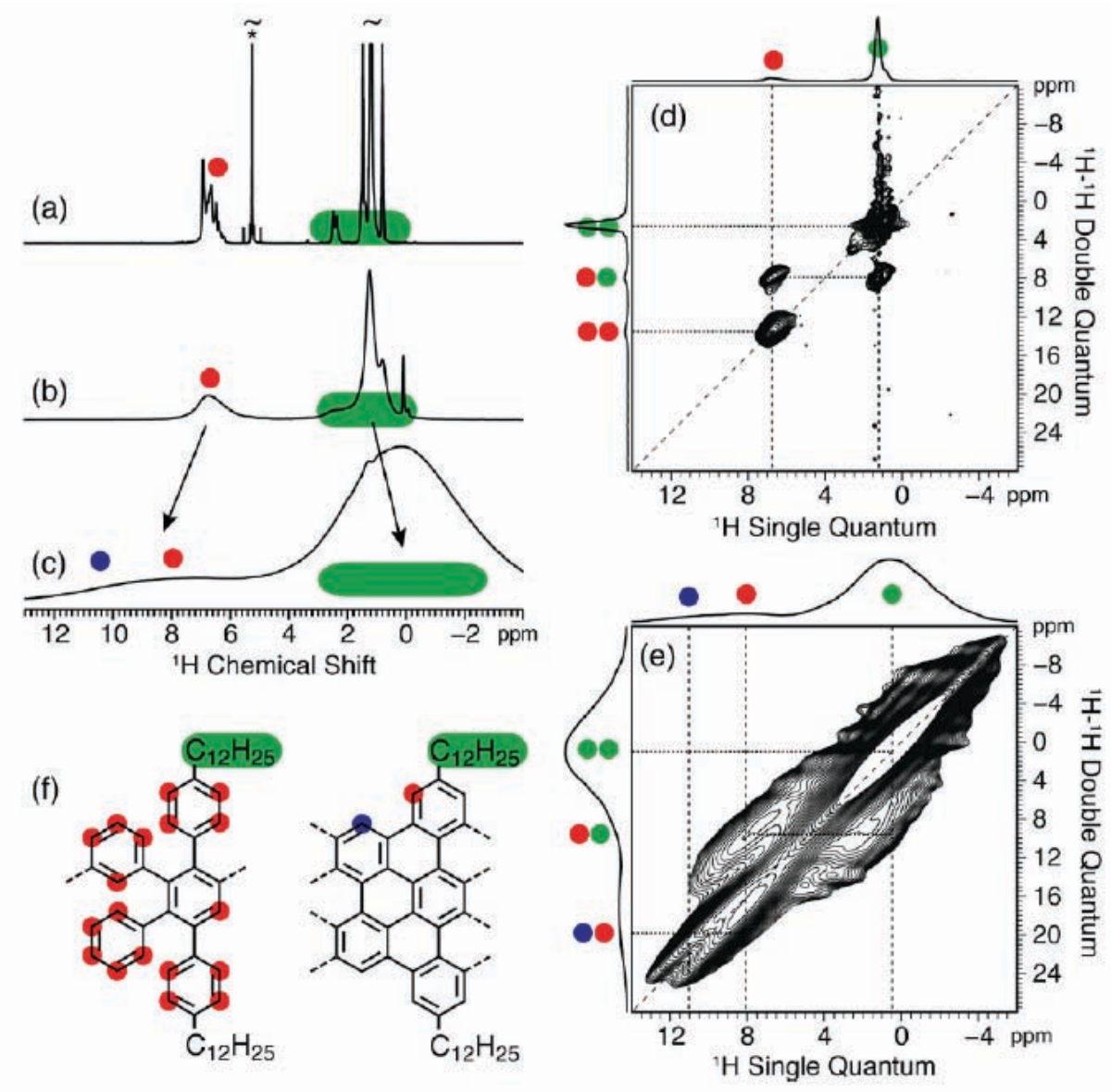

Figure 27. (a) Liquid-state $1 \mathrm{D}{ }^{1} \mathrm{H}$ spectrum of precursor dissolved in $\mathrm{CD}_{2} \mathrm{Cl}_{2}$ and $1 \mathrm{D}{ }^{1} \mathrm{H}$ MAS spectra of (b) solid precursor and (c) GNR. The solid-state precursor spectrum shows relatively narrow lines indicating a flexible structure whereas the broad resonance-shifted spectrum of GNR indicates the presence of an extended inhomogeneous packed $\pi$-conjugated system. The $2 \mathrm{D}{ }^{1} \mathrm{H}$ ${ }^{1} \mathrm{H}$ DQ-SQ correlation spectra of (d) precursor and (e) GNR probe internuclear spatial proximities. The assignment scheme is seen in (f). The liquid-state spectrum was acquired at $7 \mathrm{~T}$ and the solidstate spectra were acquired at 16.45 T using $59524 \mathrm{~Hz}$ MAS. Adapted with permission from Ref. [237]. Copyright 2014 Macmillan Publishers Limited.

?

The 2D ${ }^{1} \mathrm{H}-{ }^{1} \mathrm{H}$ DQ-SQ correlation spectrum of the precursor displayed in Figure $27 \mathrm{~d}$ shows relatively narrow resonances, and a distinct cross-correlation between the aromatic and aliphatic protons. Turning to the GNR, the spectrum in Figure 27 e displays broadened lines with resonance frequencies extending over a large range, and more delocalized cross-correlations. A relatively short $\mathrm{DQ}$ recoupling of $33.6 \mu$ s (two rotor periods using $59524 \mathrm{~Hz}$ MAS) in combination with cross-correlations only extending up to ca. $10 \mathrm{ppm}$ 
and a split ridge at higher ppm values allowed for the assignment of the high-frequency part of the split ridge to the cove protons (blue in Figure 27) and the low-frequency part to the outer protons (red in Figure 27).

\section{OUTLOOK}

Molecular order, site-specific dynamics, local packing and domain sizes are all important (sub)nanoscale level structural information in soft organic nanomaterials. As shown in this contribution, solid-state NMR spectroscopy provides a very powerful toolbox to precisely determine these details. To get a more complete picture of the material, NMR spectroscopy can easily be combined with complementary experimental and numerical techniques in a multi-technique approach. This approach holds much promise in the exploration of new hybrid nanomaterials, gel structures, polymer blends and graphenerelated materials. Other research activities, such as studying surface interactions and defects in nanomaterials, will likewise benefit from the continuing development of solidstate NMR spectroscopy techniques. One of the recent very promising advancements in the field of NMR spectroscopy is dynamic nuclear polarization (DNP). DNP has already shown great prospect in boosting the sensitivity in NMR spectroscopy, which in combination with highly sophisticated pulse sequences, appropriate sample preparation, and ongoing hardware development, will hopefully pave the way for exciting new explorations also in the field of soft organic nanomaterials. 


\section{ACKNOWLEDGEMENTS}

We gratefully acknowledge the support from the Villum Foundation under the Young Investigator Programme (VKR023122).

\section{REFERENCES}

[1] M. Möller, K. Matyjaszewski, Polymer Science: A Comprehensive Reference., Elsevier, Amsterdam, 2012.

[2] J.M. Lehn, Toward self-organization and complex matter, Science, 295 (2002) 2400-2403.

[3] J.M. Lehn, Supramolecular Chemistry-Scope and Perspectives Molecules, Supermolecules, and Molecular Devices, Angewandte Chemie International Edition, 27 (1988) 89-112.

[4] R.F. Service, How far can we push chemical self-assembly?, Science, 309 (2005) 95-95.

[5] H. Sirringhaus, P.J. Brown, R.H. Friend, M.M. Nielsen, K. Bechgaard, B.M.W. Langeveld-Voss, A.J.H. Spiering, R.A.J. Janssen, E.W. Meijer, P. Herwig, D.M. de Leeuw, Two-dimensional charge transport in self-organized, high-mobility conjugated polymers, Nature, 401 (1999) 685-688.

[6] E. Meijer, D. de Leeuw, S. Setayesh, E. van Veenendaal, B. Huisman, P. Blom, J. Hummelen, U. Scherf, T. Klapwijk, Solution-processed ambipolar organic field-effect transistors and inverters, Nature Materials, 2 (2003) 678-682.

[7] M. Watson, A. Fechtenkotter, K. Müllen, Big is beautiful - "Aromaticity" revisited from the viewpoint of macromolecular and supramolecular benzene chemistry, Chemical Reviews, 101 (2001) 1267-1300.

[8] A.D. Schlüter, Handbook of Conducting Polymers, 2nd ed., Marcel Decker, New York, 1998.

[9] M. Graetzel, R.A.J. Janssen, D.B. Mitzi, E.H. Sargent, Materials interface engineering for solution-processed photovoltaics, Nature, 488 (2012) 304-312.

[10] H.J. Dyson, P.E. Wright, Intrinsically unstructured proteins and their functions, Nature Reviews Molecular Cell Biology, 6 (2005) 197-208.

[11] C.K. Ober, S.Z.D. Cheng, P.T. Hammond, M. Muthukumar, E. Reichmanis, K.L. Wooley, T.P. Lodge, Research in Macromolecular Science: Challenges and Opportunities for the Next Decade, Macromolecules, 42 (2009) 465-471.

[12] P.V.R. Schleyer, C. Maerker, A. Dransfeld, H. Jiao, N. Hommes, Nucleus-independent chemical shifts: A simple and efficient aromaticity probe, Journal Of The American Chemical Society, 118 (1996) 6317-6318.

[13] P. Lazzeretti, Ring currents, Progress In Nuclear Magnetic Resonance Spectroscopy, 36 (2000) 1-88. 
[14] K. Schmidt-Rohr, H. Spiess, Multidimensional Solid-State NMR and Polymers, Academic Press, 1994.

[15] R. Sijbesma, F. Beijer, L. Brunsveld, B. Folmer, J. Hirschberg, R. Lange, J. Lowe, E. Meijer, Reversible polymers formed from self-complementary monomers using quadruple hydrogen bonding, Science, 278 (1997) 1601-1604.

[16] S.P. Brown, H.W. Spiess, Advanced solid-state NMR methods for the elucidation of structure and dynamics of molecular, macromolecular, and supramolecular systems, Chemical Reviews, 101 (2001) 4125-4155.

[17] M.R. Chierotti, R. Gobetto, Solid-state NMR studies of weak interactions in supramolecular systems, Chemical Communications, (2008) 1621-1634.

[18] J. Schmidt, A. Hoffmann, H.W. Spiess, D. Sebastiani, Bulk chemical shifts in hydrogen-bonded systems from first-principles calculations and solid-state-NMR, Journal Of Physical Chemistry B, 110 (2006) 23204-23210.

[19] W.L. Earl, D.L. Vanderhart, Observations in Solid Polyethylenes by C-13 Nuclear MagneticResonance with Magic Angle Sample Spinning, Macromolecules, 12 (1979) 762-767.

[20] D.L. Vanderhart, Influence of Molecular Packing on Solid-State C-13 Chemical-Shifts - the NAlkanes, Journal Of Magnetic Resonance, 44 (1981) 117-125.

[21] J. Clauss, K. Schmidt-Rohr, A. Adam, C. Boeffel, H.W. Spiess, Stiff Macromolecules with Aliphatic Side Chains: Side Chain Mobility, Conformation, and Organization from 2D Solid-state NMR Spectroscopy, Macromolecules, 25 (1992) 5208-5214.

[22] I. Ando, T. Asakura, Solid State NMR of Polymers, Elsevier, Amsterdam, 1998.

[23] I. Farnan, P.J. Grandinetti, J.H. Baltisberger, J.F. Stebbins, U. Werner, M.A. Eastman, A. Pines, Quantification of the Disorder in Network-Modified Silicate-Glasses, Nature, 358 (1992) 3135.

[24] R.E. Youngman, S.T. Haubrich, J.W. Zwanziger, M.T. Janicke, B.F. Chmelka, Short-and Intermediate-Range Structural Ordering in Glassy Boron Oxide, Science, 269 (1995) 1416-1420.

[25] N. Hedin, R. Graf, S.C. Christiansen, C. Gervais, R.C. Hayward, J. Eckert, B.F. Chmelka, Structure of a surfactant-templated silicate framework in the absence of 3D crystallinity, Journal Of The American Chemical Society, 126 (2004) 9425-9432.

[26] S. Cadars, A. Lesage, C.J. Pickard, P. Sautet, L. Emsley, Characterizing Slight Structural Disorder in Solids by Combined Solid-State NMR and First Principles Calculations, J Phys Chem A, 113 (2009) 902-911.

[27] M. Mehring, Principles of High Resolution NMR in Solids, Springer Berlin Heidelberg, 1983.

[28] M.H. Levitt, Spin Dynamics: Basics of Nuclear Magnetic Resonance, Wiley, 2008.

[29] U. Haeberlen, J.S. Waugh, Coherent averaging effects in magnetic resonance, Physical Review, 175 (1968) 453-467. 
[30] M.M. Maricq, Application of average Hamiltonian theory to the NMR of solids, Physical Review B, 25 (1982) 6622-6632.

[31] N.C. Nielsen, L.A. Strassø, A.B. Nielsen, Dipolar Recoupling, in: J.C.C. Chan (Ed.) Solid State NMR, Springer Berlin Heidelberg, 2012, pp. 1-45.

[32] M.H. Levitt, Symmetry-Based Pulse Sequences in Magic-Angle Spinning Solid-State NMR, in: eMagRes, John Wiley \& Sons, Ltd, 2007.

[33] J.H. Shirley, Solution of the Schrödinger Equation with a Hamiltonian Periodic in Time, Physical Review, 138 (1965) B979-B987.

[34] I. Scholz, J.D. van Beek, M. Ernst, Operator-based Floquet theory in solid-state NMR, Solid State Nuclear Magnetic Resonance, 37 (2010) 39-59.

[35] M. Leskes, P.K. Madhu, S. Vega, Floquet theory in solid-state nuclear magnetic resonance, Progress in Nuclear Magnetic Resonance Spectroscopy, 57 (2010) 345-380.

[36] M.H. Levitt, The Signs of Frequencies and Phases in NMR, Journal of Magnetic Resonance, 126 (1997) 164-182.

[37] M.H. Levitt, O.G. Johannessen, Signs of Frequencies and Phases in NMR: The Role of Radiofrequency Mixing, Journal of Magnetic Resonance, 142 (2000) 190-194.

[38] M.R. Hansen, R. Graf, H.W. Spiess, Solid-State NMR in Macromolecular Systems: Insights on How Molecular Entities Move, Accounts of Chemical Research, 46 (2013) 1996-2007.

[39] W. Sommer, J. Gottwald, D.E. Demco, H.W. Spiess, Dipolar Heteronuclear Multiple-Quantum NMR Spectroscopy in Rotating Solids, Journal of Magnetic Resonance, Series A, 113 (1995) 131134.

[40] M. Feike, D.E. Demco, R. Graf, J. Gottwald, S. Hafner, H.W. Spiess, Broadband MultipleQuantum NMR Spectroscopy, Journal of Magnetic Resonance, Series A, 122 (1996) 214-221.

[41] K. Saalwächter, F. Lange, K. Matyjaszewski, C.-F. Huang, R. Graf, BaBa-xy16: Robust and broadband homonuclear $\mathrm{DQ}$ recoupling for applications in rigid and soft solids up to the highest MAS frequencies, Journal of Magnetic Resonance, 212 (2011) 204-215.

[42] M.J. Bayro, M. Huber, R. Ramachandran, T.C. Davenport, B.H. Meier, M. Ernst, R.G. Griffin, Dipolar truncation in magic-angle spinning NMR recoupling experiments, The Journal of Chemical Physics, 130 (2009) 114506.

[43] C. Filip, S. Hafner, I. Schnell, D.E. Demco, H.W. Spiess, Solid-state nuclear magnetic resonance spectra of dipolar-coupled multi-spin systems under fast magic angle spinning, The Journal of Chemical Physics, 110 (1999) 423-440.

[44] T. Gullion, J. Schaefer, Rotational-echo double-resonance NMR, Journal of Magnetic Resonance (1969), 81 (1989) 196-200.

[45] H. Eckert, 40 yrs CPMAS \& 25 yrs REDOR, Solid State Nuclear Magnetic Resonance. 
[46] S.P. Brown, Recent Advances in Solid-State MAS NMR Methodology for Probing Structure and Dynamics in Polymeric and Supramolecular Systems, Macromol Rapid Comm, 30 (2009) 688716.

[47] S.P. Brown, Applications of high-resolution 1H solid-state NMR, Solid State Nuclear Magnetic Resonance, 41 (2012) 1-27.

[48] K. Saalwächter, Proton multiple-quantum NMR for the study of chain dynamics and structural constraints in polymeric soft materials, Progress in Nuclear Magnetic Resonance Spectroscopy, 51 (2007) 1-35.

[49] R.L. Johnson, J.M. Anderson, B.H. Shanks, X. Fang, M. Hong, K. Schmidt-Rohr, Spectrally edited 2D 13C13C NMR spectra without diagonal ridge for characterizing 13C-enriched lowtemperature carbon materials, Journal of Magnetic Resonance, 234 (2013) 112-124.

[50] J.D. Mao, K. Schmidt-Rohr, Methylene spectral editing in solid-state 13C NMR by three-spin coherence selection, Journal of Magnetic Resonance, 176 (2005) 1-6.

[51] J.K. Williams, K. Schmidt-Rohr, M. Hong, Aromatic spectral editing techniques for magicangle-spinning solid-state NMR spectroscopy of uniformly 13C-labeled proteins, Solid State Nuclear Magnetic Resonance, 72 (2015) 118-126.

[52] J.E. Tanner, Use of the Stimulated Echo in NMR Diffusion Studies, The Journal of Chemical Physics, 52 (1970) 2523-2526.

[53] Q. Chen, K. Schmidt-Rohr, Measurement of the local $1 \mathrm{H}$ spin-diffusion coefficient in polymers, Solid State Nuclear Magnetic Resonance, 29 (2006) 142-152.

[54] J. Clauss, K. Schmidt-Rohr, H.W. Spiess, Determination of Domain Sizes in Heterogeneous Polymers by Solid-State Nmr, Acta Polym, 44 (1993) 1-17.

[55] R.C. Nieuwendaal, H.W. Ro, D.S. Germack, R.J. Kline, M.F. Toney, C.K. Chan, A. Agrawal, D. Gundlach, D.L. VanderHart, D.M. Delongchamp, Measuring Domain Sizes and Compositional Heterogeneities in P3HT-PCBM Bulk Heterojunction Thin Films with 1H Spin Diffusion NMR Spectroscopy, Adv Funct Mater, 22 (2012) 1255-1266.

[56] T.M. Werkhoven, F.M. Mulder, C. Zune, R. Jérôme, H.J.M. de Groot, Determination of Polyisoprene-block-poly(methyl methacrylate) Domain Sizes Using $1 \mathrm{H}$ Spin Diffusion, Macromolecular Chemistry and Physics, 204 (2003) 46-51.

[57] E.R. deAzevedo, W.G. Hu, T.J. Bonagamba, K. Schmidt-Rohr, Centerband-only detection of exchange: Efficient analysis of dynamics in solids by NMR, Journal of the American Chemical Society, 121 (1999) 8411-8412.

[58] D.E. Favre, D.J. Schaefer, B.F. Chmelka, Direct determination of motional correlation times by 1D MAS and 2D exchange NMR techniques, Journal of Magnetic Resonance, 134 (1998) 261-279.

[59] M.R. Hansen, R. Graf, H.W. Spiess, Interplay of Structure and Dynamics in Functional Macromolecular and Supramolecular Systems As Revealed by Magnetic Resonance Spectroscopy, Chemical Reviews, (2015). 
[60] K. Schäler, M. Roos, P. Micke, Y. Golitsyn, A. Seidlitz, T. Thurn-Albrecht, H. Schneider, G. Hempel, K. Saalwächter, Basic principles of static proton low-resolution spin diffusion NMR in nanophase-separated materials with mobility contrast, Solid State Nuclear Magnetic Resonance, 72 (2015) 50-63.

[61] J.L. Jungnickel, J.W. Forbes, Quantitative Measurement of Hydrogen Types by Intergrated Nuclear Magnetic Resonance Intensities, Analytical Chemistry, 35 (1963) 938-942.

[62] D.P. Hollis, Quantitative Analysis of Aspirin, Phenacetin, and Caffeine Mixtures by Nuclear Magnetic Resonance Spectrometry, Analytical Chemistry, 35 (1963) 1682-1684.

[63] F. Malz, H. Jancke, Validation of quantitative NMR, Journal of Pharmaceutical and Biomedical Analysis, 38 (2005) 813-823.

[64] B.M. Fung, A.K. Khitrin, K. Ermolaev, An Improved Broadband Decoupling Sequence for Liquid Crystals and Solids, Journal of Magnetic Resonance, 142 (2000) 97-101.

[65] A. Detken, E.H. Hardy, M. Ernst, B.H. Meier, Simple and efficient decoupling in magic-angle spinning solid-state NMR: the XiX scheme, Chemical Physics Letters, 356 (2002) 298-304.

[66] R.S. Thakur, N.D. Kurur, P.K. Madhu, Swept-frequency two-pulse phase modulation for heteronuclear dipolar decoupling in solid-state NMR, Chemical Physics Letters, 426 (2006) 459463.

[67] A. Equbal, M. Bjerring, P.K. Madhu, N.C. Nielsen, A unified heteronuclear decoupling strategy for magic-angle-spinning solid-state NMR spectroscopy, The Journal of Chemical Physics, 142 (2015) 184201.

[68] A. Equbal, M. Bjerring, P.K. Madhu, N.C. Nielsen, Improving spectral resolution in biological solid-state NMR using phase-alternated rCW heteronuclear decoupling, Chemical Physics Letters, 635 (2015) 339-344.

[69] R.R. Ernst, W.A. Anderson, Application of Fourier Transform Spectroscopy to Magnetic Resonance, Review of Scientific Instruments, 37 (1966) 93-102.

[70] I.P. Gerothanassis, Methods of avoiding the effects of acoustic ringing in pulsed fourier transform nuclear magnetic resonance spectroscopy, Progress in Nuclear Magnetic Resonance Spectroscopy, 19 (1987) 267-329.

[71] D.G. Cory, W.M. Ritchey, Suppression of signals from the probe in bloch decay spectra, Journal of Magnetic Resonance (1969), 80 (1988) 128-132.

[72] A.C. Kunwar, G.L. Turner, E. Oldfield, Solid-state spin-echo Fourier transform NMR of 39K and 67Zn salts at high field, Journal of Magnetic Resonance (1969), 69 (1986) 124-127.

[73] Q. Chen, S.S. Hou, K. Schmidt-Rohr, A simple scheme for probehead background suppression in one-pulse 1H NMR, Solid State Nuclear Magnetic Resonance, 26 (2004) 11-15.

[74] S. Zhang, X. Wu, M. Mehring, Elimination of ringing effects in multiple-pulse sequences, Chemical Physics Letters, 173 (1990) 481-484. 
[75] C. Jaeger, F. Hemmann, EASY: A simple tool for simultaneously removing background, deadtime and acoustic ringing in quantitative NMR spectroscopy-Part I: Basic principle and applications, Solid State Nuclear Magnetic Resonance, 57-58 (2014) 22-28.

[76] C. Jaeger, F. Hemmann, "EASY: A simple tool for simultaneously removing background, deadtime and acoustic ringing in quantitative NMR spectroscopy. Part II: Improved ringing suppression, application to quadrupolar nuclei, cross polarisation and 2D NMR", Solid State Nuclear Magnetic Resonance, 63-64 (2014) 13-19.

[77] M. Robin Bendall, R.E. Gordon, Depth and refocusing pulses designed for multipulse NMR with surface coils, Journal of Magnetic Resonance (1969), 53 (1983) 365-385.

[78] W. Koźmiński, K. Jackowski, Application of adiabatic inversion pulses for elimination of baseline distortions in Fourier transform NMR. A natural abundance 170 NMR spectrum for gaseous acetone, Magnetic Resonance in Chemistry, 38 (2000) 459-462.

[79] S.P. Brown, Probing proton-proton proximities in the solid state, Progress in Nuclear Magnetic Resonance Spectroscopy, 50 (2007) 199-251.

[80] K. Saalwächter, Robust NMR Approaches for the Determination of Homonuclear DipoleDipole Coupling Constants in Studies of Solid Materials and Biomolecules, ChemPhysChem, 14 (2013) 3000-3014.

[81] K. Saalwächter, P. Ziegler, O. Spyckerelle, B. Haidar, A. Vidal, J.-U. Sommer, 1H multiplequantum nuclear magnetic resonance investigations of molecular order distributions in poly(dimethylsiloxane) networks: Evidence for a linear mixing law in bimodal systems, The Journal of Chemical Physics, 119 (2003) 3468-3482.

[82] L. Jasinska-Walc, M.R. Hansen, D. Dudenko, A. Rozanski, M. Bouyahyi, M. Wagner, R. Graf, R. Duchateau, Topological behavior mimicking ethylene-hexene copolymers using branched lactones and macrolactones, Polymer Chemistry, 5 (2014) 3306-3310.

[83] H. Geen, J.J. Titman, J. Gottwald, H.W. Spiess, Solid-state proton multiple-quantum NMR spectroscopy with fast magic angle spinning, Chemical Physics Letters, 227 (1994) 79-86.

[84] J. Gottwald, D.E. Demco, R. Graf, H.W. Spiess, High-resolution double-quantum NMR spectroscopy of homonuclear spin pairs and proton connectivities in solids, Chemical Physics Letters, 243 (1995) 314-323.

[85] H.W. Spiess, Double-quantum NMR Spectroscopy of Dipolar-coupled Spins under Fast Magicangle Spinning, in: eMagRes, John Wiley \& Sons, Ltd, 2007.

[86] M.R. Hansen, R. Graf, S. Sekharan, D. Sebastiani, Columnar Packing Motifs of Functionalized Perylene Derivatives: Local Molecular Order Despite Long-Range Disorder, Journal of the American Chemical Society, 131 (2009) 5251-5256.

[87] C. Ochsenfeld, S.P. Brown, I. Schnell, J. Gauss, H.W. Spiess, Structure Assignment in the Solid State by the Coupling of Quantum Chemical Calculations with NMR Experiments: A Columnar Hexabenzocoronene Derivative, Journal of the American Chemical Society, 123 (2001) 2597-2606. 
[88] A. Pines, M.G. Gibby, J.S. Waugh, Proton-enhanced nuclear induction spectroscopy. a method for high resolution nmr of dilute spins in solids, The Journal of Chemical Physics, 56 (1972) 1776-1777.

[89] A. Pines, M.G. Gibby, J.S. Waugh, Proton-enhanced NMR of dilute spins in solids, The Journal of Chemical Physics, (1973) 569-590.

[90] W. Kolodziejski, J. Klinowski, Kinetics of Cross-Polarization in Solid-State NMR: A Guide for Chemists, Chemical Reviews, 102 (2002) 613-628.

[91] S.R. Hartmann, E.L. Hahn, Nuclear double resonance in the rotating frame, Physical Review, 128 (1962) 2042-2053.

[92] G. Metz, X.L. Wu, S.O. Smith, Ramped-Amplitude Cross-Polarization in Magic-Angle Spinning NMR, Journal Of Magnetic Resonance Series A, 110 (1994) 219-227.

[93] A. Nowacka, N.A. Bongartz, O.H.S. Ollila, T. Nylander, D. Topgaard, Signal intensities in 1H13C CP and INEPT MAS NMR of liquid crystals, Journal of Magnetic Resonance, 230 (2013) 165175.

[94] A. Nowacka, S. Douezan, L. Wadso, D. Topgaard, E. Sparr, Small polar molecules like glycerol and urea can preserve the fluidity of lipid bilayers under dry conditions, Soft Matter, 8 (2012) 1482-1491.

[95] A. Nowacka, P.C. Mohr, J. Norrman, R.W. Martin, D. Topgaard, Polarization Transfer SolidState NMR for Studying Surfactant Phase Behavior, Langmuir, 26 (2010) 16848-16856.

[96] G.A. Morris, R. Freeman, Enhancement of nuclear magnetic resonance signals by polarization transfer [18], Journal of the American Chemical Society, 101 (1979) 760-762.

[97] A. Bohle, G. Brunklaus, M.R. Hansen, T.W. Schleuss, A.F.M. Kilbinger, J. Seltmann, H.W. Spiess, Hydrogen-Bonded Aggregates of Oligoaramide-Poly(ethylene glycol) Block Copolymers, Macromolecules, 43 (2010) 4978-4985.

[98] B.D. Olsen, R.A. Segalman, Structure and Thermodynamics of Weakly Segregated Rod-Coil Block Copolymers, Macromolecules, 38 (2005) 10127-10137.

[99] H.-A. Klok, J.F. Langenwalter, S. Lecommandoux, Self-Assembly of Peptide-Based Diblock Oligomers, Macromolecules, 33 (2000) 7819-7826.

[100] B.D. Olsen, R.A. Segalman, Phase Transitions in Asymmetric Rod-Coil Block Copolymers, Macromolecules, 39 (2006) 7078-7083.

[101] B. de Boer, U. Stalmach, P.F. van Hutten, C. Melzer, V.V. Krasnikov, G. Hadziioannou, Supramolecular self-assembly and opto-electronic properties of semiconducting block copolymers, Polymer, 42 (2001) 9097-9109.

[102] N.C. Nielsen, H. Bildso/e, H.J. Jakobsen, M.H. Levitt, Double-quantum homonuclear rotary resonance: Efficient dipolar recovery in magic-angle spinning nuclear magnetic resonance, The Journal of Chemical Physics, 101 (1994) 1805-1812. 
[103] S. Laage, J.R. Sachleben, S. Steuernagel, R. Pierattelli, G. Pintacuda, L. Emsley, Fast acquisition of multi-dimensional spectra in solid-state NMR enabled by ultra-fast MAS, Journal of Magnetic Resonance, 196 (2009) 133-141.

[104] S. Laage, A. Marchetti, J. Sein, R. Pierattelli, H.J. Sass, S. Grzesiek, A. Lesage, G. Pintacuda, L. Emsley, Band-Selective 1H-13C Cross-Polarization in Fast Magic Angle Spinning Solid-State NMR Spectroscopy, Journal of the American Chemical Society, 130 (2008) 1721617217.

[105] B.H. Meier, Cross polarization under fast magic angle spinning: thermodynamical considerations, Chemical Physics Letters, 188 (1992) 201-207.

[106] V. Ladizhansky, S. Vega, A Method for Measuring Heteronuclear (1H-13C) Distances in High Speed MAS NMR, Journal of the American Chemical Society, 122 (2000) 3465-3472.

[107] M. Hong, X. Yao, K. Jakes, D. Huster, Investigation of Molecular Motions by Lee-Goldburg Cross-Polarization NMR Spectroscopy, The Journal of Physical Chemistry B, 106 (2002) 73557364.

[108] M. Lee, W.I. Goldburg, Nuclear-Magnetic-Resonance Line Narrowing by a Rotating rf Field, Physical Review, 140 (1965) A1261-A1271.

[109] A. Bielecki, A.C. Kolbert, M.H. Levitt, Frequency-switched pulse sequences: Homonuclear decoupling and dilute spin NMR in solids, Chemical Physics Letters, 155 (1989) 341-346.

[110] A. Bielecki, A.C. Kolbert, H.J.M. De Groot, R.G. Griffin, M.H. Levitt, Frequency-Switched Lee-Goldburg Sequences in Solids, in: W. Warren S (Ed.) Advances in Magnetic and Optical Resonance, Academic Press, 1990, pp. 111-124.

[111] E. Vinogradov, P.K. Madhu, S. Vega, High-resolution proton solid-state NMR spectroscopy by phase-modulated Lee-Goldburg experiment, Chemical Physics Letters, 314 (1999) 443-450.

[112] B. Alonso, D. Massiot, Multi-scale NMR characterisation of mesostructured materials using $1 \mathrm{H} \rightarrow 13 \mathrm{C}$ through-bond polarisation transfer, fast MAS, and $1 \mathrm{H}$ spin diffusion, Journal of Magnetic Resonance, 163 (2003) 347-352.

[113] R.L. Johnson, K. Schmidt-Rohr, Quantitative solid-state 13C NMR with signal enhancement by multiple cross polarization, Journal of Magnetic Resonance, 239 (2014) 44-49.

[114] O.D. Bernardinelli, M.A. Lima, C.A. Rezende, I. Polikarpov, E.R. deAzevedo, Quantitative 13C MultiCP solid-state NMR as a tool for evaluation of cellulose crystallinity index measured directly inside sugarcane biomass, Biotechnology for Biofuels, 8 (2015) 1-11.

[115] M. Carravetta, M. Edén, X. Zhao, A. Brinkmann, M.H. Levitt, Symmetry principles for the design of radiofrequency pulse sequences in the nuclear magnetic resonance of rotating solids, Chemical Physics Letters, 321 (2000) 205-215.

[116] X. Zhao, M. Edén, M.H. Levitt, Recoupling of heteronuclear dipolar interactions in solid-state NMR using symmetry-based pulse sequences, Chemical Physics Letters, 342 (2001) 353-361. 
[117] M. Sardo, R. Siegel, S.M. Santos, J. Rocha, J.R.B. Gomes, L. Mafra, Combining Multinuclear High-Resolution Solid-State MAS NMR and Computational Methods for Resonance Assignment of Glutathione Tripeptide, The Journal of Physical Chemistry A, 116 (2012) 6711-6719.

[118] L. Mafra, S.M. Santos, R. Siegel, I. Alves, F.A. Almeida Paz, D. Dudenko, H.W. Spiess, Packing Interactions in Hydrated and Anhydrous Forms of the Antibiotic Ciprofloxacin: a SolidState NMR, X-ray Diffraction, and Computer Simulation Study, Journal of the American Chemical Society, 134 (2012) 71-74.

[119] A. Böckmann, M. Ernst, B.H. Meier, Spinning proteins, the faster, the better?, Journal of Magnetic Resonance, 253 (2015) 71-79.

[120] A. Lesage, D. Sakellariou, S. Hediger, B. Eléna, P. Charmont, S. Steuernagel, L. Emsley, Experimental aspects of proton NMR spectroscopy in solids using phase-modulated homonuclear dipolar decoupling, Journal of Magnetic Resonance, 163 (2003) 105-113.

[121] B. Elena, G. de Paëpe, L. Emsley, Direct spectral optimisation of proton-proton homonuclear dipolar decoupling in solid-state NMR, Chemical Physics Letters, 398 (2004) 532-538.

[122] X.L. Yao, K. Schmidt-Rohr, M. Hong, Medium- and Long-Distance 1H-13C Heteronuclear Correlation NMR in Solids, Journal of Magnetic Resonance, 149 (2001) 139-143.

[123] E. Salager, J.-N. Dumez, R.S. Stein, S. Steuernagel, A. Lesage, B. Elena-Herrmann, L. Emsley, Homonuclear dipolar decoupling with very large scaling factors for high-resolution ultrafast magic angle spinning $1 \mathrm{H}$ solid-state NMR spectroscopy, Chemical Physics Letters, 498 (2010) 214-220.

[124] F. Etzold, I.A. Howard, N. Forler, D.M. Cho, M. Meister, H. Mangold, J. Shu, M.R. Hansen, K. Müllen, F. Laquai, The Effect of Solvent Additives on Morphology and Excited-State Dynamics in PCPDTBT:PCBM Photovoltaic Blends, Journal of the American Chemical Society, 134 (2012) 10569-10583.

[125] J. Peet, J.Y. Kim, N.E. Coates, W.L. Ma, D. Moses, A.J. Heeger, G.C. Bazan, Efficiency enhancement in low-bandgap polymer solar cells by processing with alkane dithiols, Nature Materials, 6 (2007) 497-500.

[126] A. Lesage, D. Sakellariou, S. Steuernagel, L. Emsley, Carbon-Proton Chemical Shift Correlation in Solid-State NMR by Through-Bond Multiple-Quantum Spectroscopy, Journal of the American Chemical Society, 120 (1998) 13194-13201.

[127] A. Bax, R.H. Griffey, B.L. Hawkins, Correlation of proton and nitrogen-15 chemical shifts by multiple quantum NMR, Journal of Magnetic Resonance (1969), 55 (1983) 301-315.

[128] M.A.R. Meier, J.O. Metzger, U.S. Schubert, Plant oil renewable resources as green alternatives in polymer science, Chemical Society Reviews, 36 (2007) 1788-1802.

[129] F. Fenouillot, A. Rousseau, G. Colomines, R. Saint-Loup, J.P. Pascault, Polymers from renewable 1,4:3,6-dianhydrohexitols (isosorbide, isomannide and isoidide): A review, Progress in Polymer Science, 35 (2010) 578-622.

[130] P. Gallezot, Conversion of biomass to selected chemical products, Chemical Society Reviews, 41 (2012) 1538-1558. 
[131] H. Mutlu, R. Hofsäß, R.E. Montenegro, M.A.R. Meier, Self-metathesis of fatty acid methyl esters: full conversion by choosing the appropriate plant oil, Rsc Advances, 3 (2013) 4927-4934.

[132] N.Q. Ran, L.S. Zhao, Z.M. Chen, J.H. Tao, Recent applications of biocatalysis in developing green chemistry for chemical synthesis at the industrial scale, Green Chem, 10 (2008) 361-372.

[133] H.M. Konig, A.F.M. Kilbinger, Learning from nature: beta-sheet-mimicking copolymers get organized, Angewandte Chemie-International Edition, 46 (2007) 8334-8340.

[134] A. Bohle, G. Brunklaus, M.R. Hansen, T.W. Schleuss, A.F.M. Kilbinger, J. Seltmann, H.W. Spiess, Hydrogen-Bonded Aggregates of Oligoaramide-Poly(ethylene glycol) Block Copolymers, Macromolecules, 43 (2010) 4978-4985.

[135] M. Wegner, D. Dudenko, D. Sebastiani, A.R.A. Palmans, T.F.A. de Greef, R. Graf, H.W. Spiess, The impact of the amide connectivity on the assembly and dynamics of benzene-1,3,5tricarboxamides in the solid state, Chemical Science, 2 (2011) 2040-2049.

[136] J. Shu, D. Dudenko, M. Esmaeili, J.H. Park, S.R. Puniredd, J.Y. Chang, D.W. Breiby, W. Pisula, M.R. Hansen, Coexistence of Helical Morphologies in Columnar Stacks of Star-Shaped Discotic Hydrazones, Journal of the American Chemical Society, 135 (2013) 11075-11086.

[137] J. Lu, P.A. Mirau, A.E. Tonelli, Chain conformations and dynamics of crystalline polymers as observed in their inclusion compounds by solid-state NMR, Progress in Polymer Science, 27 (2002) 357-401.

[138] M.P.F. Pepels, M.R. Hansen, H. Goossens, R. Duchateau, Macromolecules, 46 (2013) 7668-7677.

[139] R.H. Grubbs, Olefin-metathesis catalysts for the preparation of molecules and materials (Nobel lecture), Angewandte Chemie-International Edition, 45 (2006) 3760-3765.

[140] M. Kamigaito, T. Ando, M. Sawamoto, Metal-catalyzed living radical polymerization, Chemical Reviews, 101 (2001) 3689-3745.

[141] S. Monsaert, A.L. Vila, R. Drozdzak, P. Van Der Voort, F. Verpoort, Latent olefin metathesis catalysts, Chemical Society Reviews, 38 (2009) 3360-3372.

[142] T.W. Baughman, K.B. Wagener, Recent advances in ADMET polymerization, in: M.R. Buchmeiser (Ed.) Metathesis Polymerization, 2005, pp. 1-42.

[143] Y.Y. Wei, R. Graf, J.C. Sworen, C.Y. Cheng, C.R. Bowers, K.B. Wagener, H.W. Spiess, Local and Collective Motions in Precise Polyolefins with Alkyl Branches: A Combination of $\mathrm{H}-2$ and $\mathrm{C}-13$ Solid-State NMR Spectroscopy, Angewandte Chemie-International Edition, 48 (2009) 4617-4620.

[144] R.G. Alamo, K. Jeon, R.L. Smith, E. Boz, K.B. Wagener, M.R. Bockstaller, Crystallization of Polyethylenes Containing Chlorines: Precise vs Random Placement, Macromolecules, 41 (2008) 7141-7151.

[145] C.J. Luo, S.D. Stoyanov, E. Stride, E. Pelan, M. Edirisinghe, Electrospinning versus fibre production methods: from specifics to technological convergence, Chemical Society Reviews, 41 (2012) 4708-4735. 
[146] L. Jasinska-Walc, M. Villani, D. Dudenko, O. van Asselen, E. Klop, S. Rastogi, M.R. Hansen, C.E. Koning, Local Conformation and Cocrystallization Phenomena in Renewable DiaminoisoidideBased Polyamides Studied by FT-IR, Solid State NMR, and WAXD, Macromolecules, 45 (2012) 2796-2808.

[147] L. Jasinska-Walc, D. Dudenko, A. Rozanski, S. Thiyagarajan, P. Sowinski, D. van Es, J. Shu, M.R. Hansen, C.E. Koning, Structure and Molecular Dynamics in Renewable Polyamides from Dideoxy-Diamino Isohexide, Macromolecules, 45 (2012) 5653-5666.

[148] J. Wu, L. Jasinska-Walc, D. Dudenko, A. Rozanski, M.R. Hansen, D. van Es, C.E. Koning, An Investigation of Polyamides Based on Isoidide-2,5-dimethyleneamine as a Green Rigid Building Block with Enhanced Reactivity, Macromolecules, 45 (2012) 9333-9346.

[149] G. Flèche, M. Huchette, Starch/Stärke, 38 (1986) 26-30.

[150] A. Gitsas, G. Floudas, M. Mondeshki, I. Lieberwirth, H.W. Spiess, H. latrou, N. Hadjichristidis, A. Hirao, Hierarchical Self-Assembly and Dynamics of a Miktoarm Star chimera Composed of Poly(gamma-benzyl-L-glutamate), Polystyrene, and Polyisoprene, Macromolecules, 43 (2010) 1874-1881.

[151] C. Holland, F. Vollrath, A.J. Ryan, O.O. Mykhaylyk, Silk and Synthetic Polymers: Reconciling 100 Degrees of Separation, Advanced Materials, 24 (2012) 105-+.

[152] F.G. Omenetto, D.L. Kaplan, New Opportunities for an Ancient Material, Science, 329 (2010) 528-531.

[153] D. Porter, J. Guan, F. Vollrath, Spider Silk: Super Material or Thin Fibre?, Advanced Materials, 25 (2013) 1275-1279.

[154] B. Lotz, F.C. Cesari, Chemical-Structure and the Crystalline-Structures of Bombyx-Mori Silk Fibroin, Biochimie, 61 (1979) 205-214.

[155] T. Kameda, Y. Tamada, Variable-temperature C-13 solid-state NMR study of the molecular structure of honeybee wax and silk, Int J Biol Macromol, 44 (2009) 64-69.

[156] A.A. Walker, S. Weisman, T. Kameda, T.D. Sutherland, Natural Templates for Coiled-Coil Biomaterials from Praying Mantis Egg Cases, Biomacromolecules, 13 (2012) 4264-4272.

[157] T. Kameda, Quantifying the fraction of alanine residues in an alpha-helical conformation in hornet silk using solid-state NMR, Polym J, 44 (2012) 876-881.

[158] J.B. Addison, N.N. Ashton, W.S. Weber, R.J. Stewart, G.P. Holland, J.L. Yarger, beta-Sheet Nanocrystalline Domains Formed from Phosphorylated Serine-Rich Motifs in Caddisfly Larval Silk: A Solid State NMR and XRD Study, Biomacromolecules, 14 (2013) 1140-1148.

[159] J.B. Addison, T.M.O. Popp, W.S. Weber, J.S. Edgerly, G.P. Holland, J.L. Yarger, Structural characterization of nanofiber silk produced by embiopterans (webspinners), Rsc Advances, 4 (2014) 41301-41313.

[160] H. Saito, R. Tabeta, T. Asakura, Y. Iwanaga, A. Shoji, T. Ozaki, I. Ando, High-Resolution C$13 \mathrm{Nmr}$-Study of Silk Fibroin in the Solid-State by the Cross-Polarization Magic Angle Spinning 
Method - Conformational Characterization of Silk-I and Silk-li Type Forms of Bombyx-Mori Fibroin by the Conformation-Dependent C-13 Chemical-Shifts, Macromolecules, 17 (1984) 1405-1412.

[161] I. Marcotte, J.D. van Beek, B.H. Meier, Molecular disorder and structure of spider dragline silk investigated by two-dimensional solid-state NMR spectroscopy, Macromolecules, 40 (2007) 19952001.

[162] G.P. Holland, J.E. Jenkins, M.S. Creager, R.V. Lewis, J.L. Yarger, Solid-state NMR investigation of major and minor ampullate spider silk in the native and hydrated states, Biomacromolecules, 9 (2008) 651-657.

[163] T. Izdebski, P. Akhenblit, J.E. Jenkins, J.L. Yarger, G.P. Holland, Structure and Dynamics of Aromatic Residues in Spider Silk: 2D Carbon Correlation NMR of Dragline Fibers, Biomacromolecules, 11 (2010) 168-174.

[164] C.A. Michal, L.W. Jelinski, Rotational-echo double-resonance in complex biopolymers: a study of Nephila clavipes dragline silk, J Biomol Nmr, 12 (1998) 231-241.

[165] T. Asakura, J.M. Yao, T. Yamane, K. Umemura, A.S. Ulrich, Heterogeneous structure of silk fibers from Bombyx mori resolved by $\mathrm{C}-13$ solid-state NMR spectroscopy, Journal of the American Chemical Society, 124 (2002) 8794-8795.

[166] T. Asakura, J. Ashida, T. Yamane, T. Kameda, Y. Nakazawa, K. Ohgo, K. Komatsu, A repeated beta-turn structure in poly(Ala-Gly) as a model for silk I of Bombyx mori silk fibroin studied with two-dimensional spin-diffusion NMR under off magic angle spinning and rotational echo double resonance, J Mol Biol, 306 (2001) 291-305.

[167] T. Asakura, K. Ohgo, T. Ishida, P. Taddei, P. Monti, R. Kishore, Possible implications of serine and tyrosine residues and intermolecular interactions on the appearance of silk I structure of Bombyx mori silk fibroin-derived synthetic peptides: High-resolution C-13 cross-polarization/magicangle spinning NMR study, Biomacromolecules, 6 (2005) 468-474.

[168] T. Gullion, R. Kishore, T. Asakura, Determining dihedral angles and local structure in silk peptide by C-13-H-2 REDOR, Journal of the American Chemical Society, 125 (2003) 7510-7511.

[169] R.E. Marsh, R.B. Corey, L. Pauling, An Investigation of the Structure of Silk Fibroin, Biochim Biophys Acta, 16 (1955) 1-34.

[170] Y. Takahashi, M. Gehoh, K. Yuzuriha, Structure refinement and diffuse streak scattering of silk (Bombyx mori), Int J Biol Macromol, 24 (1999) 127-138.

[171] T. Asakura, Y. Suzuki, Y. Nakazawa, G.P. Holland, J.L. Yarger, Elucidating silk structure using solid-state NMR, Soft Matter, 9 (2013) 11440-11450.

[172] R.V. Lewis, Spider Silk - the Unraveling of a Mystery, Accounts of Chemical Research, 25 (1992) 392-398.

[173] P.M. Cunniff, S.A. Fossey, M.A. Auerbach, J.W. Song, D.L. Kaplan, W.W. Adams, E. R.K., D. Mahoney, D.L. Vezie, Mechanical and thermal properties of dragline silk from the spider Nephila clavipes, Polym. Adv. Technol., 5 (1994) 401-410. 
[174] J.E. Jenkins, M.S. Creager, E.B. Butler, R.V. Lewis, J.L. Yarger, G.P. Holland, Solid-state NMR evidence for elastin-like beta-turn structure in spider dragline silk, Chemical Communications, 46 (2010) 6714-6716.

[175] A.H. Simmons, C.A. Michal, L.W. Jelinski, Molecular orientation and two-component nature of the crystalline fraction of spider dragline silk, Science, 271 (1996) 84-87.

[176] M.B. Hinman, R.V. Lewis, Isolation of a Clone Encoding a 2nd Dragline Silk Fibroin - NephilaClavipes Dragline Silk Is a 2-Protein Fiber, J Biol Chem, 267 (1992) 19320-19324.

[177] M. Xu, R.V. Lewis, Structure of a Protein Superfiber - Spider Dragline Silk, Proceedings of the National Academy of Sciences of the United States of America, 87 (1990) 7120-7124.

[178] G.P. Holland, M.S. Creager, J.E. Jenkins, R.V. Lewis, J.L. Yarger, Determining secondary structure in spider dragline silk by carbon-carbon correlation solid-state NMR spectroscopy, Journal of the American Chemical Society, 130 (2008) 9871-9877.

[179] H.R. Kricheldorf, D. Muller, Secondary Structure of Peptides .3. C-13 Nmr Cross Polarization Magic Angle Spinning Spectroscopic Characterization of Solid Polypeptides, Macromolecules, 16 (1983) 615-623.

[180] H.R. Kricheldorf, D. Muller, Secondary Structure of Peptides .15. C-13 Nmr Cp Mas Study of Solid Elastin and Proline-Containing Copolyesters, Int J Biol Macromol, 6 (1984) 145-151.

[181] L.G. Pease, M.H. Frey, S.J. Opella, Observation of Conformationally Distinct Proline Residues in 2 Cyclic-Peptides by Solid-State Nuclear Magnetic-Resonance, Journal of the American Chemical Society, 103 (1981) 467-468.

[182] M.S. Creager, J.E. Jenkins, L.A. Thagard-Yeaman, A.E. Brooks, J.A. Jones, R.V. Lewis, G.P. Holland, J.L. Yarger, Solid-State NMR Comparison of Various Spiders' Dragline Silk Fiber, Biomacromolecules, 11 (2010) 2039-2043.

[183] Y. Liu, A. Sponner, D. Porter, F. Vollrath, Proline and processing of spider silks, Biomacromolecules, 9 (2008) 116-121.

[184] P.M. Beaujuge, J.R. Reynolds, Color Control in pi-Conjugated Organic Polymers for Use in Electrochromic Devices, Chemical Reviews, 110 (2010) 268-320.

[185] P.M. Beaujuge, J.M.J. Frechet, Molecular Design and Ordering Effects in pi-Functional Materials for Transistor and Solar Cell Applications, Journal of the American Chemical Society, 133 (2011) 20009-20029.

[186] Y.L. Guo, G. Yu, Y.Q. Liu, Functional Organic Field-Effect Transistors, Advanced Materials, 22 (2010) 4427-4447.

[187] H. Sirringhaus, 25th Anniversary Article: Organic Field-Effect Transistors: The Path Beyond Amorphous Silicon, Advanced Materials, 26 (2014) 1319-1335.

[188] K.S. Yook, J.Y. Lee, Organic Materials for Deep Blue Phosphorescent Organic Light-Emitting Diodes, Advanced Materials, 24 (2012) 3169-3190. 
[189] C.M. Zhong, C.H. Duan, F. Huang, H.B. Wu, Y. Cao, Materials and Devices toward Fully Solution Processable Organic Light-Emitting Diodes, Chemistry of Materials, 23 (2011) 326-340.

[190] E. Cantatore, Applications of organic and printed electronics : a technology-enabled revolution, Springer, New York, 2013.

[191] J. Rivnay, S.C.B. Mannsfeld, C.E. Miller, A. Salleo, M.F. Toney, Quantitative Determination of Organic Semiconductor Microstructure from the Molecular to Device Scale, Chemical Reviews, 112 (2012) 5488-5519.

[192] V. Coropceanu, J. Cornil, D.A. da Silva, Y. Olivier, R. Silbey, J.L. Bredas, Charge transport in organic semiconductors, Chemical Reviews, 107 (2007) 926-952.

[193] C. Goh, R.J. Kline, M.D. McGehee, E.N. Kadnikova, J.M.J. Frechet, Molecular-weightdependent mobilities in regioregular poly(3-hexyl-thiophene) diodes, Applied Physics Letters, 86 (2005).

[194] R.S. Loewe, S.M. Khersonsky, R.D. McCullough, A simple method to prepare head-to-tail coupled, regioregular poly(3-alkylthiophenes) using grignard metathesis, Advanced Materials, 11 (1999) 250-+.

[195] D. Dudenko, A. Kiersnowski, J. Shu, W. Pisula, D. Sebastiani, H.W. Spiess, M.R. Hansen, A Strategy for Revealing the Packing in Semicrystalline $\pi$-Conjugated Polymers: Crystal Structure of Bulk Poly-3-hexyl-thiophene (P3HT), Angewandte Chemie International Edition, 51 (2012) 1106811072.

[196] J. Warnan, C. Cabanetos, A. El Labban, M.R. Hansen, C. Tassone, M.F. Toney, P.M. Beaujuge, Ordering Effects in Benzo[1,2-b:4,5-b ']difuran-thieno[3,4-c]pyrrole-4,6-dione Polymers with > 7\% Solar Cell Efficiency, Advanced Materials, 26 (2014) 4357-4362.

[197] W.R. Mateker, T. Heumueller, R. Cheacharoen, I.T. Sachs-Quintana, M.D. McGehee, Molecular Packing and Arrangement Govern the Photo-Oxidative Stability of Organic Photovoltaic Materials, Chemistry of Materials, 27 (2015) 6345-6353.

[198] D.M. DeLongchamp, R.J. Kline, A. Herzing, Nanoscale structure measurements for polymerfullerene photovoltaics, Energy \& Environmental Science, 5 (2012) 5980-5993.

[199] F. Laquai, Y.S. Park, J.J. Kim, T. Basche, Excitation Energy Transfer in Organic Materials: From Fundamentals to Optoelectronic Devices, Macromol Rapid Comm, 30 (2009) 1203-1231.

[200] J.J.M. Halls, K. Pichler, R.H. Friend, S.C. Moratti, A.B. Holmes, Exciton diffusion and dissociation in a poly(p-phenylenevinylene)/C-60 heterojunction photovoltaic cell, Applied Physics Letters, 68 (1996) 3120-3122.

[201] A. Haugeneder, M. Neges, C. Kallinger, W. Spirkl, U. Lemmer, J. Feldmann, U. Scherf, E. Harth, A. Gugel, K. Mullen, Exciton diffusion and dissociation in conjugated polymer fullerene blends and heterostructures, Physical Review B, 59 (1999) 15346-15351.

[202] D.E. Markov, E. Amsterdam, P.W.M. Blom, A.B. Sieval, J.C. Hummelen, Accurate measurement of the exciton diffusion length in a conjugated polymer using a heterostructure with a side-chain cross-linked fullerene layer, J Phys Chem A, 109 (2005) 5266-5274. 
[203] E. Mena-Osteritz, A. Meyer, B.M.W. Langeveld-Voss, R.A.J. Janssen, E.W. Meijer, P. Bauerle, Two-dimensional crystals of poly(3-alkylthiophene)s: Direct visualization of polymer folds in submolecular resolution, Angewandte Chemie-International Edition, 39 (2000) 2680-2684.

[204] X.N. Yang, J. Loos, S.C. Veenstra, W.J.H. Verhees, M.M. Wienk, J.M. Kroon, M.A.J. Michels, R.A.J. Janssen, Nanoscale morphology of high-performance polymer solar cells, Nano Lett, 5 (2005) 579-583.

[205] A. Buda, D.E. Demco, M. Bertmer, B. Blumich, B. Reining, H. Keul, H. Hocker, Domain sizes in heterogeneous polymers by spin diffusion using single-quantum and double-quantum dipolar filters, Solid State Nuclear Magnetic Resonance, 24 (2003) 39-67.

[206] S.S. Hou, Q. Chen, K. Schmidt-Rohr, Two-dimensional C-13 NMR with H-1 spin diffusion for characterizing domain sizes in unlabeled polymers, Macromolecules, 37 (2004) 1999-2001.

[207] W. Lequieu, P. Van de Velde, F.E. Du Prez, P. Adriaensens, L. Storme, J. Gelan, Solid state NMR study of segmented polymer networks: fine-tuning of phase morphology via their molecular design, Polymer, 45 (2004) 7943-7951.

[208] S. Chambon, R. Mens, K. Vandewal, E. Clodic, M. Scharber, L. Lutsen, J. Gelan, J. Manca, D. Vanderzande, P. Adriaensens, Influence of octanedithiol on the nanomorphology of PCPDTBT:PCBM blends studied by solid-state NMR, Sol Energ Mat Sol C, 96 (2012) 210-217.

[209] R.C. Nieuwendaal, C.R. Snyder, R.J. Kline, E.K. Lin, D.L. VanderHart, D.M. DeLongchamp, Measuring the Extent of Phase Separation in Poly-3-Hexylthiophene/Phenyl-C-61-Butyric Acid Methyl Ester Photovoltaic Blends with H-1 Spin Diffusion NMR Spectroscopy, Chemistry of Materials, 22 (2010) 2930-2936.

[210] K.R. Graham, C. Cabanetos, J.P. Jahnke, M.N. Idso, A. El Labban, G.O.N. Ndjawa, T. Heumueller, K. Vandewal, A. Salleo, B.F. Chmelka, A. Amassian, P.M. Beaujuge, M.D. McGehee, Importance of the Donor:Fullerene Intermolecular Arrangement for High-Efficiency Organic Photovoltaics, Journal of the American Chemical Society, 136 (2014) 9608-9618.

[211] I. Mcculloch, M. Heeney, C. Bailey, K. Genevicius, M. I, M. Shkunov, D. Sparrowe, S. Tierney, R. Wagner, W.M. Zhang, M.L. Chabinyc, R.J. Kline, M.D. Mcgehee, M.F. Toney, Liquidcrystalline semiconducting polymers with high charge-carrier mobility, Nature Materials, 5 (2006) 328-333.

[212] N.C. Cates, R. Gysel, Z. Beiley, C.E. Miller, M.F. Toney, M. Heeney, I. McCulloch, M.D. McGehee, Tuning the Properties of Polymer Bulk Heterojunction Solar Cells by Adjusting Fullerene Size to Control Intercalation, Nano Lett, 9 (2009) 4153-4157.

[213] N.C. Miller, E. Cho, M.J.N. Junk, R. Gysel, C. Risko, D. Kim, S. Sweetnam, C.E. Miller, L.J. Richter, R.J. Kline, M. Heeney, I. McCulloch, A. Amassian, D. Acevedo-Feliz, C. Knox, M.R. Hansen, D. Dudenko, B.F. Chmelka, M.F. Toney, J.L. Bredas, M.D. McGehee, Use of X-Ray Diffraction, Molecular Simulations, and Spectroscopy to Determine the Molecular Packing in a Polymer-Fullerene Bimolecular Crystal, Advanced Materials, 24 (2012) 6071-+.

[214] A.K. Geim, K.S. Novoselov, The rise of graphene, Nat Mater, 6 (2007) 183-191. 
[215] K.S. Novoselov, A.K. Geim, S.V. Morozov, D. Jiang, Y. Zhang, S.V. Dubonos, I.V. Grigorieva, A.A. Firsov, Electric Field Effect in Atomically Thin Carbon Films, Science, 306 (2004) 666-669.

[216] K.S. Novoselov, D. Jiang, F. Schedin, T.J. Booth, V.V. Khotkevich, S.V. Morozov, A.K. Geim, Two-dimensional atomic crystals, Proceedings of the National Academy of Sciences of the United States of America, 102 (2005) 10451-10453.

[217] S. Yang, R.E. Bachman, X. Feng, K. Müllen, Use of Organic Precursors and Graphenes in the Controlled Synthesis of Carbon-Containing Nanomaterials for Energy Storage and Conversion, Accounts of Chemical Research, 46 (2013) 116-128.

[218] M. Pumera, Graphene-based nanomaterials for energy storage, Energy \& Environmental Science, 4 (2011) 668-674.

[219] Y. Zhu, S. Murali, M.D. Stoller, K.J. Ganesh, W. Cai, P.J. Ferreira, A. Pirkle, R.M. Wallace, K.A. Cychosz, M. Thommes, D. Su, E.A. Stach, R.S. Ruoff, Carbon-Based Supercapacitors Produced by Activation of Graphene, Science, 332 (2011) 1537-1541.

[220] S. Bae, H. Kim, Y. Lee, X. Xu, J.-S. Park, Y. Zheng, J. Balakrishnan, T. Lei, H. Ri Kim, Y.I. Song, Y.-J. Kim, K.S. Kim, B. Ozyilmaz, J.-H. Ahn, B.H. Hong, S. lijima, Roll-to-roll production of 30-inch graphene films for transparent electrodes, Nat Nano, 5 (2010) 574-578.

[221] Y. Wang, X. Chen, Y. Zhong, F. Zhu, K.P. Loh, Large area, continuous, few-layered graphene as anodes in organic photovoltaic devices, Applied Physics Letters, 95 (2009) 063302.

[222] F. Schwierz, Graphene transistors, Nat Nano, 5 (2010) 487-496.

[223] X. Wan, Y. Huang, Y. Chen, Focusing on Energy and Optoelectronic Applications: A Journey for Graphene and Graphene Oxide at Large Scale, Accounts of Chemical Research, 45 (2012) 598-607.

[224] J.E. Cochran, M.J.N. Junk, A.M. Glaudell, P.L. Miller, J.S. Cowart, M.F. Toney, C.J. Hawker, B.F. Chmelka, M.L. Chabinyc, Molecular Interactions and Ordering in Electrically Doped Polymers: Blends of PBTTT and F4TCNQ, Macromolecules, 47 (2014) 6836-6846.

[225] M. Mehring, H. Weber, W. Müller, G. Wegner, High resolution 13C NMR in undoped cis- and trans-polyacetylene, Solid State Communications, 45 (1983) 1079-1082.

[226] K. Parvez, Z.-S. Wu, R. Li, X. Liu, R. Graf, X. Feng, K. Müllen, Exfoliation of Graphite into Graphene in Aqueous Solutions of Inorganic Salts, Journal of the American Chemical Society, 136 (2014) 6083-6091.

[227] W. Cai, R.D. Piner, F.J. Stadermann, S. Park, M.A. Shaibat, Y. Ishii, D. Yang, A. Velamakanni, S.J. An, M. Stoller, J. An, D. Chen, R.S. Ruoff, Synthesis and Solid-State NMR Structural Characterization of 13C-Labeled Graphite Oxide, Science, 321 (2008) 1815-1817.

[228] Y. Ishii, 13C-13C dipolar recoupling under very fast magic angle spinning in solid-state nuclear magnetic resonance: Applications to distance measurements, spectral assignments, and high-throughput secondary-structure determination, The Journal of Chemical Physics, 114 (2001) 8473-8483. 
[229] T. Szabó, O. Berkesi, P. Forgó, K. Josepovits, Y. Sanakis, D. Petridis, I. Dékány, Evolution of Surface Functional Groups in a Series of Progressively Oxidized Graphite Oxides, Chemistry of Materials, 18 (2006) 2740-2749.

[230] A. Lerf, H. He, M. Forster, J. Klinowski, Structure of Graphite Oxide Revisited, The Journal of Physical Chemistry B, 102 (1998) 4477-4482.

[231] S. Park, Y. Hu, J.O. Hwang, E.-S. Lee, L.B. Casabianca, W. Cai, J.R. Potts, H.-W. Ha, S. Chen, J. Oh, S.O. Kim, Y.-H. Kim, Y. Ishii, R.S. Ruoff, Chemical structures of hydrazine-treated graphene oxide and generation of aromatic nitrogen doping, Nat Commun, 3 (2012) 638.

[232] K.A. Ritter, J.W. Lyding, The influence of edge structure on the electronic properties of graphene quantum dots and nanoribbons, Nat Mater, 8 (2009) 235-242.

[233] Y.-W. Son, M.L. Cohen, S.G. Louie, Half-metallic graphene nanoribbons, Nature, 444 (2006) 347-349.

[234] A.H. Castro Neto, F. Guinea, N.M.R. Peres, K.S. Novoselov, A.K. Geim, The electronic properties of graphene, Reviews of Modern Physics, 81 (2009) 109-162.

[235] X. Li, X. Wang, L. Zhang, S. Lee, H. Dai, Chemically Derived, Ultrasmooth Graphene Nanoribbon Semiconductors, Science, 319 (2008) 1229-1232.

[236] S. Osella, A. Narita, M.G. Schwab, Y. Hernandez, X. Feng, K. Müllen, D. Beljonne, Graphene Nanoribbons as Low Band Gap Donor Materials for Organic Photovoltaics: Quantum Chemical Aided Design, ACS Nano, 6 (2012) 5539-5548.

[237] A. Narita, X. Feng, Y. Hernandez, S.A. Jensen, M. Bonn, H. Yang, I.A. Verzhbitskiy, C. Casiraghi, M.R. Hansen, A.H.R. Koch, G. Fytas, O. Ivasenko, B. Li, K.S. Mali, T. Balandina, S. Mahesh, S. De Feyter, K. Müllen, Synthesis of structurally well-defined and liquid-phaseprocessable graphene nanoribbons, Nat Chem, 6 (2014) 126-132.

[238] A. Narita, I.A. Verzhbitskiy, W. Frederickx, K.S. Mali, S.A. Jensen, M.R. Hansen, M. Bonn, S. De Feyter, C. Casiraghi, X. Feng, K. Müllen, Bottom-Up Synthesis of Liquid-Phase-Processable Graphene Nanoribbons with Near-Infrared Absorption, ACS Nano, 8 (2014) 11622-11630. 